\title{
Cauchy theory for the gravity water waves system with non-localized initial data
}

\author{
T. Alazard ${ }^{\mathrm{a}, *, 1}$, N. Burq ${ }^{\mathrm{b}, \mathrm{a}}$, C. Zuily ${ }^{\mathrm{b}}$ \\ a Département de Mathématiques et Applications, UMR 8553 du CNRS, École Normale Supérieure, 45, rue d'Ulm, 75005 Paris Cedex, France \\ b Laboratoire de Mathématiques d'Orsay, UMR 8628 du CNRS, Université Paris-Sud, 91405 Orsay Cedex, France
}

Received 20 March 2014; received in revised form 13 October 2014; accepted 16 October 2014

Available online 11 November 2014

\begin{abstract}
In this article, we develop the local Cauchy theory for the gravity water waves system, for rough initial data which do not decay at infinity. We work in the context of $L^{2}$-based uniformly local Sobolev spaces introduced by Kato [22]. We prove a classical well-posedness result (without loss of derivatives). Our result implies also a local well-posedness result in Hölder spaces (with loss of $d / 2$ derivatives). As an illustration, we solve a question raised by Boussinesq in [9] on the water waves problem in a canal. We take benefit of an elementary observation to show that the strategy suggested in [9] does indeed apply to this setting.

๑ 2014 L'Association Publications de l'Institut Henri Poincaré. Published by Elsevier B.V. All rights reserved.
\end{abstract}

Keywords: Water-waves; Cauchy problem; Uniformly local Sobolev spaces; Paradifferential calculus

\section{Introduction}

We are interested in this paper in the free boundary problem describing the motion of an incompressible, irrotational fluid flow moving under the force of gravitation, without surface tension, in case where the initial data are neither localized nor periodic. There are indeed two cases where the mathematical analysis is rather well understood: firstly for periodic initial data (in the classical Sobolev spaces $H^{s}\left(\mathbf{T}^{d}\right)$ ) and secondly when they are decaying to zero at infinity (for instance for data in $H^{s}\left(\mathbf{R}^{d}\right)$ with $s$ large enough). With regards to the analysis of the Cauchy problem, we refer to the recent papers of Lannes [25], Wu [31,32] and Germain, Masmoudi and Shatah [19]. We also refer to the introduction of [2] or [7,10,12,23,26,30,33] for more references. However, one can think to the moving surface of a lake or a canal where the waves are neither periodic nor decaying to zero (see also [16]).

A most natural strategy would be to solve the Cauchy problem in the classical Hölder spaces $W^{k, \infty}\left(\mathbf{R}^{d}\right)$. However even the linearized system at the origin (the fluid at rest) is ill-posed in these spaces (see Remark 2.4 below), and this strategy leads consequently to loss of derivatives. Having this loss of derivatives in mind, the other natural

\footnotetext{
* Corresponding author.

E-mail address: alazard@dma.ens.fr (T. Alazard).

1 T.A. was supported by the French Agence Nationale de la Recherche, projects ANR-08-JCJC-0132-01 and ANR-08-JCJC-0124-01.
} 
approach is to work in the framework of $L^{2}$ based uniformly local Sobolev spaces, denoted by $H_{u l}^{s}\left(\mathbf{R}^{d}\right)$. These spaces were introduced by Kato (see [22]) in the analysis of hyperbolic systems. Notice however, that compared to general hyperbolic systems, the water waves system appears to be non-local, which induces new difficulties. This framework appears to be quite natural in our context. Indeed, the uniformly local Sobolev spaces $H_{u l}^{s}\left(\mathbf{R}^{d}\right)$ contain, in particular, the usual Sobolev spaces $H^{s}\left(\mathbf{R}^{d}\right)$, the periodic Sobolev spaces $H^{s}\left(\mathbf{T}^{d}\right)$ (spaces of periodic functions on $\mathbf{R}^{d}$ ), the sum $H^{s}\left(\mathbf{R}^{d}\right)+H^{s}\left(\mathbf{T}^{d}\right)$ and also the Hölder spaces $W^{s, \infty}\left(\mathbf{R}^{d}\right)$ (and as a by-product of our analysis, we get well-posedness in Hölder spaces, with a loss of derivatives).

The aim of this paper is precisely to prove that the water waves system is locally (in time) well posed in the framework of uniformly local Sobolev spaces. Moreover, following our previous paper [2], the data for which we solve the Cauchy problem are allowed to be quite rough. Indeed we shall assume, for instance, that the initial free surface is the graph of a function which belongs to the space $H_{u l}^{s+\frac{1}{2}}\left(\mathbf{R}^{d}\right)$ for $s>1+\frac{d}{2}$. In particular, in terms of Sobolev embedding, the initial free surface is merely $W^{\frac{3}{2}, \infty}\left(\mathbf{R}^{d}\right)$ thus may have unbounded curvature. On the other hand this threshold should be compared with the scaling of the problem. Indeed it is known that the water wave system has a scaling invariance for which the critical space for the initial free surface is the space $\dot{H}^{1+\frac{d}{2}}\left(\mathbf{R}^{d}\right)$ (or $W^{1, \infty}\left(\mathbf{R}^{d}\right)$ ). This shows that we solve here the Cauchy problem for data $\frac{1}{2}$ above the scaling. (Notice that in [3] we prove well-posedness, in the classical Sobolev spaces, $\frac{1}{2}-\frac{1}{12}$ above the scaling when $d \geq 2$ and $\frac{1}{2}-\frac{1}{24}$ when $d=1$.)

As an illustration of the relevance of this low regularity Cauchy theory in the context of local spaces, we solve a question raised by Boussinesq in 1910 [9] on the water waves problem in a canal. In [9], Boussinesq suggested to reduce the water-waves system in a canal to the same system on $\mathbf{R}^{3}$ with periodic conditions with respect to one variable, by a simple reflection/periodization procedure (with respect to the normal variable to the boundary of the canal). However, this idea remained inapplicable for the simple reason that the even extension of a smooth function on the half line is in general merely Lipschitz continuous (due to the singularity at the origin). As a consequence, even if one starts with a smooth initial domain, the reflected/periodized domain will only be Lipschitz continuous. Here, we are able to take benefit of an elementary (though seemingly previously unnoticed) observation which shows that actually, as soon as we are looking for reasonably smooth solutions, the angle between the free surface and the vertical boundary of the canal is a right angle. Consequently, the reflected/periodized domain enjoys additional smoothness (namely up to $C^{3}$ ), which is enough to apply our rough data Cauchy theory and to show that the strategy suggested in [9] does indeed apply. This appears to be the first result on Cauchy theory for the water-wave system in a domain with boundary.

The present paper relies on the strategies developed in our previous paper [2] and we follow the same scheme of proof. In Section 7, we develop the machinery of para-differential calculus in the framework of uniformly local spaces that we need later. We think that this section could be useful for further studies in this framework. In Section 3 we prove that the Dirichlet-Neumann operator is well defined in this framework (notice that this fact is not straightforward, see $[18,15]$ for related works), and we give a precise description (including sharp elliptic estimates in very rough domains) on these spaces. In Section 4, we symmetrize the system and prove a priori estimates. In Section 5, we prove contraction estimates and well posedness. In Section 6, we give the application to the canal (and swimming pools). Finally, in Appendix A, we prove that in the context of Hölder spaces, the water-waves system linearized on the trivial solution (rest) is ill posed.

\section{The problem and the result}

In this paper we shall denote by $t \in \mathbf{R}$ the time variable and by $x \in \mathbf{R}^{d}$ (where $d \geq 1$ ), $y \in \mathbf{R}$, the horizontal and vertical space variables. We work in a fluid domain with free boundary and fixed bottom of the form

$$
\begin{aligned}
& \Omega=\left\{(t, x, y) \in[0, T] \times \mathbf{R}^{d} \times \mathbf{R}:(x, y) \in \Omega(t)\right\} \quad \text { where } \\
& \Omega(t)=\left\{(x, y) \in \mathbf{R}^{d} \times \mathbf{R}: \eta_{*}(x)<y<\eta(t, x)\right\} .
\end{aligned}
$$

Here the free surface is described by $\eta$, an unknown of the problem, and the bottom by a given function $\eta_{*}$. We shall only assume that $\eta_{*}$ is bounded and continuous. We assume that the bottom is the graph of a function for the sake of simplicity: our analysis applies whenever one has the Poincaré inequality given by Lemma 3.1 below. In the case without bottom, the Dirichlet Neumann operator in the simplest case of a flat interface $(\eta=0)$ is equal to $\left|D_{x}\right|$. It is 
possible (though not completely trivial due to the 0 frequency singularity) to show that this operator acts on uniformly local spaces. It is hence very likely that our result should hold also in the case without bottom, but this would require additional material and we preferred not to pursue in this direction.

We shall denote by $\Sigma$ the free surface and by $\Gamma$ the bottom,

$$
\begin{aligned}
& \Sigma=\left\{(t, x, y) \in[0, T] \times \mathbf{R}^{d} \times \mathbf{R}:(x, y) \in \Sigma(t)\right\} \quad \text { where } \\
& \Sigma(t)=\left\{(x, y) \in \mathbf{R}^{d} \times \mathbf{R}: y=\eta(t, x)\right\}, \\
& \Gamma=\left\{(x, y) \in \mathbf{R}^{d} \times \mathbf{R}: y=\eta_{*}(x)\right\} .
\end{aligned}
$$

We shall use the following notations

$$
\nabla_{x}=\left(\partial_{x_{i}}\right)_{1 \leq i \leq d}, \quad \nabla_{x, y}=\left(\nabla_{x}, \partial_{y}\right), \quad \Delta_{x}=\sum_{1 \leq i \leq d} \partial_{x_{i}}^{2}, \quad \Delta_{x, y}=\Delta_{x}+\partial_{y}^{2}
$$

\subsection{The equations}

The Eulerian velocity $v: \Omega \rightarrow \mathbf{R}^{d+1}$ solves the incompressible and irrotational Euler equation

$$
\partial_{t} v+\left(v \cdot \nabla_{x, y}\right) v+\nabla_{x, y} P=-g e_{y}, \quad \operatorname{div}_{x, y} v=0, \quad \operatorname{curl}_{x, y} v=0 \quad \text { in } \Omega
$$

where $g>0$ is the acceleration of the gravity, $e_{y}$ the vector $(x=0, y=1)$ and $P$ the pressure. The problem is then given by three boundary conditions:

- a kinematic condition (which states that the free surface moves with the fluid)

$$
\partial_{t} \eta=\sqrt{1+\left|\nabla_{x} \eta\right|^{2}}(v \cdot n) \quad \text { on } \Sigma,
$$

where $n$ denotes the unit normal vector to $\Sigma$,

- a dynamic condition (that expresses a balance of forces across the free surface)

$$
P=0 \quad \text { on } \Sigma,
$$

- the "solid wall" boundary condition on the bottom $\Gamma$

$$
v \cdot v=0 \text { on } \Gamma,
$$

where $v$ denotes the normal vector to $\Gamma$ whenever it exists.

Since the motion is incompressible and irrotational there exists a velocity potential $\phi: \Omega \rightarrow \mathbf{R}$ such that $v=\nabla_{x, y} \phi$, thus $\Delta_{x, y} \phi=0$ in $\Omega$. We shall work with the Zakharov/Craig-Sulem formulation of the water waves equations. We introduce

$$
\psi(t, x)=\phi(t, x, \eta(t, x))
$$

and the Dirichlet-Neumann operator

$$
\begin{aligned}
G(\eta) \psi & =\sqrt{1+\left|\nabla_{x} \eta\right|^{2}}\left(\left.\frac{\partial \phi}{\partial n}\right|_{\Sigma}\right) \\
& =\left(\partial_{y} \phi\right)(t, x, \eta(t, x))-\nabla_{x} \eta(t, x) \cdot\left(\nabla_{x} \phi\right)(t, x, \eta(t, x)) .
\end{aligned}
$$

Then (see [14] or [11]) the water waves system can be written in terms of the unknown $\eta, \psi$ as

$$
\left\{\begin{array}{l}
\partial_{t} \eta=G(\eta) \psi, \\
\partial_{t} \psi=-\frac{1}{2}\left|\nabla_{x} \psi\right|^{2}+\frac{1}{2} \frac{\left(\nabla_{x} \eta \cdot \nabla_{x} \psi+G(\eta) \psi\right)^{2}}{1+\left|\nabla_{x} \eta\right|^{2}}-g \eta .
\end{array}\right.
$$

It is useful to introduce the vertical and horizontal components of the velocity. We set 


$$
\left\{\begin{array}{l}
B=\left.\left(v_{y}\right)\right|_{\Sigma}=\frac{\nabla_{x} \eta \cdot \nabla_{x} \psi+G(\eta) \psi}{1+\left|\nabla_{x} \eta\right|^{2}}, \\
V=\left.\left(v_{x}\right)\right|_{\Sigma}=\nabla_{x} \psi-B \nabla_{x} \eta .
\end{array}\right.
$$

We recall also that the Taylor coefficient defined by $a=-\left.\frac{\partial P}{\partial y}\right|_{\Sigma}$ can be defined in terms of $\eta, \psi, B, V$ only (see Section 7.2 below and $\S 4.3 .1$ in [23]).

\subsection{The uniformly local Sobolev spaces}

We recall here the definition of the uniformly local Sobolev spaces introduced by Kato in [22].

Recall that there exists $\chi \in C^{\infty}\left(\mathbf{R}^{d}\right)$ with supp $\chi \subset[-1,1]^{d}, \chi=1$ near $\left[-\frac{1}{2}, \frac{1}{2}\right]^{d}$ such that

$$
\sum_{q \in \mathbf{Z}^{d}} \chi_{q}(x)=1, \quad \forall x \in \mathbf{R}^{d}
$$

where

$$
\chi_{q}(x)=\chi(x-q) .
$$

Definition 2.1. For $s \in \mathbf{R}$ the space $H_{u l}^{s}\left(\mathbf{R}^{d}\right)$ is the space of distributions $u \in H_{l o c}^{s}\left(\mathbf{R}^{d}\right)$ such that

$$
\|u\|_{H_{u l}^{s}\left(\mathbf{R}^{d}\right)}:=\sup _{q \in \mathbf{Z}^{d}}\left\|\chi_{q} u\right\|_{H^{s}\left(\mathbf{R}^{d}\right)}<+\infty .
$$

Endowed with this norm $H_{u l}^{s}\left(\mathbf{R}^{d}\right)$ is a Banach space. Moreover its definition is independent of the choice of the function $\chi$ in $C_{0}^{\infty}\left(\mathbf{R}^{d}\right)$ satisfying (2.3) (see Lemma 7.1 below).

Proposition 2.2. One has the following embeddings:

(1) If $s>\frac{d}{2}$ and $s-\frac{d}{2} \notin \mathbf{N}, H_{u l}^{s}\left(\mathbf{R}^{d}\right)$ is continuously embedded in $W^{s-\frac{d}{2}, \infty}\left(\mathbf{R}^{d}\right)$.

(2) If $m \in \mathbf{N}, W^{m, \infty}\left(\mathbf{R}^{d}\right)$ is continuously embedded in $H_{u l}^{m}\left(\mathbf{R}^{d}\right)$.

(3) If $s \geq 0, W^{s+\varepsilon, \infty}\left(\mathbf{R}^{d}\right)$ is continuously embedded in $H_{u l}^{s}\left(\mathbf{R}^{d}\right)$ for $\varepsilon>0$.

\subsection{The main result}

The goal of this article is to prove the following result.

Theorem 2.3. Let $d \geq 1, s>1+\frac{d}{2}$. Assume that $\eta_{*}$ is a bounded continuous function on $\mathbf{R}^{d}$. Consider an initial data $\left(\eta_{0}, \psi_{0}\right)$ satisfying the following conditions

(i) $\eta_{0} \in H_{u l}^{s+\frac{1}{2}}\left(\mathbf{R}^{d}\right), \psi_{0} \in H_{u l}^{s+\frac{1}{2}}\left(\mathbf{R}^{d}\right), V_{0} \in H_{u l}^{s}\left(\mathbf{R}^{d}\right), B_{0} \in H_{u l}^{s}\left(\mathbf{R}^{d}\right)$,

(ii) there exists $h>0$ such that $\eta_{0}(x)-\eta_{*}(x) \geq 2 h, \forall x \in \mathbf{R}^{d}$,

(iii) there exists $c>0$ such that $a_{0}(x) \geq c, \forall x \in \mathbf{R}^{d}$,

where $a_{0}$ denotes the Taylor coefficient at time $t=0$.

Then there exists $T>0$ such that the Cauchy problem for the system (2.1) with initial data $\left(\eta_{0}, \psi_{0}\right)$ at $t=0$ has a unique solution

$$
(\eta, \psi) \in L^{\infty}\left([0, T], H_{u l}^{s+\frac{1}{2}}\left(\mathbf{R}^{d}\right) \times H_{u l}^{s+\frac{1}{2}}\left(\mathbf{R}^{d}\right)\right)
$$

such that

1. $(V, B) \in L^{\infty}\left([0, T], H_{u l}^{s}\left(\mathbf{R}^{d}\right) \times H_{u l}^{s}\left(\mathbf{R}^{d}\right)\right)$,

2. $\eta(t, x)-\eta_{*}(x) \geq h, \forall(t, x) \in[0, T] \times \mathbf{R}^{d}$, 
3. $a(t, x) \geq \frac{1}{2} c, \forall(t, x) \in[0, T] \times \mathbf{R}^{d}$,

4. for any $s^{\prime}<s$,

$$
(\eta, \psi, V, B) \in C^{0}\left([0, T], H_{u l}^{s^{\prime}+\frac{1}{2}}\left(\mathbf{R}^{d}\right) \times H_{u l}^{s^{\prime}+\frac{1}{2}}\left(\mathbf{R}^{d}\right) \times H_{u l}^{s^{\prime}}\left(\mathbf{R}^{d}\right) \times H_{u l}^{s^{\prime}}\left(\mathbf{R}^{d}\right)\right) .
$$

\section{Remark 2.4.}

- Theorem 2.3 implies local well posedness in Hölder spaces: indeed, writing $H^{s}=H^{s}\left(\mathbf{R}^{d}\right), W^{s, \infty}=W^{s, \infty}\left(\mathbf{R}^{d}\right)$ and assuming that

$$
\begin{aligned}
\left(\eta_{0}, \psi_{0}, V_{0}, B_{0}\right) & \in W^{\sigma+\frac{1}{2}+\varepsilon, \infty} \times W^{\sigma+\frac{1}{2}+\varepsilon, \infty} \times W^{\sigma+\varepsilon, \infty} \times W^{\sigma+\varepsilon, \infty} \\
& \subset H_{u l}^{\sigma+\frac{1}{2}+\frac{\varepsilon}{2}} \times H_{u l}^{\sigma+\frac{1}{2}+\frac{\varepsilon}{2}} \times H_{u l}^{\sigma+\frac{\varepsilon}{2}} \times H_{u l}^{\sigma+\frac{\varepsilon}{2}}
\end{aligned}
$$

for some $\sigma>1+d / 2$ and $\varepsilon>0$, then we get a solution

$$
\begin{aligned}
(\eta, \psi, V, B) & \in C^{0}\left([0, T], H_{u l}^{\sigma+\frac{1}{2}+\frac{\varepsilon}{2}} \times H_{u l}^{\sigma+\frac{1}{2}+\frac{\varepsilon}{2}} \times H_{u l}^{\sigma+\frac{\varepsilon}{2}} \times H_{u l}^{\sigma+\frac{\varepsilon}{2}}\right) \\
& \subset C^{0}\left([0, T], W^{\sigma+\frac{1}{2}-\frac{d}{2}+\frac{\varepsilon}{2}, \infty} \times W^{\sigma+\frac{1}{2}-\frac{d}{2}+\frac{\varepsilon}{2}, \infty} \times W^{\sigma-\frac{d}{2}+\frac{\varepsilon}{2}, \infty} \times W^{\sigma-\frac{d}{2}+\frac{\varepsilon}{2}, \infty}\right) .
\end{aligned}
$$

- It is very likely that this loss of $d / 2$ derivatives cannot be completely avoided. To explain this, we begin by recalling that for $\sigma \in \mathbf{R}$ the Zygmund space $C_{*}^{\sigma}\left(\mathbf{R}^{d}\right)$ is defined, by means of a Littlewood-Paley decomposition, as the space of tempered distributions $u$ such that

$$
\left\|\Delta_{j} u\right\|_{L^{\infty}\left(\mathbf{R}^{d}\right)} \leq C 2^{-j \sigma}, \quad \forall j \geq-1 .
$$

Recall also that the linearized water waves equation around the zero solution can be written as

$$
\partial_{t} u+i\left|D_{x}\right|^{\frac{1}{2}} u=0 .
$$

The solution of this equation, with initial data $u_{0}$, is given by

$$
u(t)=S(t) u_{0}, \quad S(t)=\exp \left(-i t\left|D_{x}\right|^{\frac{1}{2}}\right) .
$$

Proposition A.1 shows that for $t \neq 0$ the operator $S(t)$ is not bounded from the Zygmund space $C_{*}^{\sigma}\left(\mathbf{R}^{d}\right)$ to $C_{*}^{S}\left(\mathbf{R}^{d}\right)$ if $s>\sigma-\frac{d}{4}$, remembering that $C_{*}^{\sigma}\left(\mathbf{R}^{d}\right)=W^{\sigma, \infty}\left(\mathbf{R}^{d}\right)$ if $\sigma \geq 0, \sigma \notin \mathbf{N}$. (For positive results see Fefferman and Stein $\left[17\right.$, p. 160].) Thus even in the linear case we have a loss of $\frac{d}{4}$ derivative.

- The result in Appendix A also shows that, in the presence of surface tension, a similar well posedness result in the framework of uniformly local Sobolev space is rather unlikely to hold. Indeed, in the presence of surface tension, the linearized operator around the solution $(\eta, \psi)=(0,0)$ can be written (see [1]) with $u=|D|^{\frac{1}{2}} \eta+i \psi$ as

$$
\partial_{t} u+i\left|D_{x}\right|^{\frac{3}{2}} u=0,\left.\quad u\right|_{t=0}=u_{0} .
$$

According to Proposition A.1 the loss of derivatives in $x$ from $u_{0}$ to the solution $u(t, \cdot), t \neq 0$, is at least $\frac{3 d}{4}$ whereas an analogue of the above theorem would give a loss of at most $\frac{d}{2}$.

- The above theorem is not contained nor contains entirely the analogue result proved in [2] in the framework of classical Sobolev spaces.

- The assumption that $\psi_{0} \in H_{u l}^{s+\frac{1}{2}}$ should be replaced by the more natural assumption $\nabla_{x} \psi_{0} \in H_{u l}^{s-\frac{1}{2}}$. However, this improvement would again require a few more developments and we preferred to keep this simpler assumption.

\section{The Dirichlet-Neumann operator}

\subsection{Definition of the Dirichlet-Neumann operator}

For $d \geq 1$ we set

$$
\left\{\begin{array}{l}
\Omega=\left\{(x, y) \in \mathbf{R}^{d+1}: \eta_{*}(x)<y<\eta(x)\right\}, \\
\Sigma=\left\{(x, y) \in \mathbf{R}^{d+1}: y=\eta(x)\right\}
\end{array}\right.
$$


where $\eta_{*}$ is a fixed bounded continuous function on $\mathbf{R}^{d}$ and $\eta \in W^{1, \infty}\left(\mathbf{R}^{d}\right)$. We shall assume that there exists $h>0$ such that

$$
\left\{(x, y) \in \mathbf{R}^{d+1}: \eta(x)-h \leq y<\eta(x)\right\} \subset \Omega .
$$

In [2] the Dirichlet-Neumann operator $G(\eta)$ associated to $\Omega$ has been defined as a continuous operator from $H^{\frac{1}{2}}\left(\mathbf{R}^{d}\right)$ to $H^{-\frac{1}{2}}\left(\mathbf{R}^{d}\right)$. Our aim here is to prove that it has a unique extension to the space $H_{u l}^{\frac{1}{2}}\left(\mathbf{R}^{d}\right)$ (see Theorem 3.8 below). Define first the space $H_{u l}^{1}(\Omega)$ by

$$
u \in H_{u l}^{1}(\Omega) \quad \Leftrightarrow \quad\|u\|_{H_{u l}^{1}(\Omega)}:=\sup _{q \in \mathbf{Z}^{d}}\left\|\chi_{q} u\right\|_{H^{1}(\Omega)}<+\infty .
$$

Each element $u \in H_{u l}^{1}(\Omega)$ has a trace on $\Sigma$ (see below) which will be denoted by $\gamma_{0} u$. We introduce the subspace $H_{u l}^{1,0}(\Omega) \subset H_{u l}^{1}(\Omega)$ defined by

$$
H_{u l}^{1,0}(\Omega)=\left\{u \in H_{u l}^{1}(\Omega): \gamma_{0} u=0\right\} .
$$

Then we have the following Poincaré inequality.

Lemma 3.1. There exists $C>0$ depending on $\|\eta\|_{L^{\infty}\left(\mathbf{R}^{d}\right)}+\left\|\eta_{*}\right\|_{L^{\infty}\left(\mathbf{R}^{d}\right)}$ such that for $\alpha \in C_{0}^{\infty}\left(\mathbf{R}^{d}\right)$ non-negative and $u \in H_{u l}^{1,0}(\Omega)$ we have

$$
\iint_{\Omega} \alpha(x)|u(x, y)|^{2} d x d y \leq C \iint_{\Omega} \alpha(x)\left|\partial_{y} u(x, y)\right|^{2} d x d y .
$$

Proof. Let $u \in H_{u l}^{1,0}(\Omega)$. It is easy to see that there exists a sequence $\left(u_{n}\right)$ of functions which are $C^{1}$ in $\Omega$ and vanish near the top boundary $y=\eta(x)$ such that

$$
\lim _{n \rightarrow+\infty}\left\|u_{n}-u\right\|_{H^{1}(\Omega \cap\{|x| \leq K\})}=0 .
$$

As a consequence, it is enough to prove the result for such functions. Let $\alpha \in C_{0}^{\infty}\left(\mathbf{R}^{d}\right), \alpha \geq 0$. We can write

$$
u(x, y)=\int_{\eta(x)}^{y} \partial_{s} u(x, s) d s
$$

from which we deduce

$$
\alpha(x)|u(x, y)|^{2} \leq\left\|\eta-\eta_{*}\right\|_{L^{\infty}} \alpha(x) \int_{\eta_{*}(x)}^{\eta(x)}\left|\partial_{s} u(x, s)\right|^{2} d s .
$$

Integrating this inequality on $\Omega$ we obtain

$$
\iint_{\Omega} \alpha(x)|u(x, y)|^{2} d x d y \leq\left\|\eta-\eta_{*}\right\|_{L^{\infty}} \iint_{\Omega} \alpha(x)\left|\partial_{y} u(x, y)\right|^{2} d x d y .
$$

\section{Remark 3.2. Let}

$$
H^{1,0}(\Omega)=\left\{u \in L^{2}(\Omega): \nabla_{x, y} u \in L^{2}(\Omega), \text { and }\left.u\right|_{y=\eta(x)}=0\right\},
$$

then we also have the Poincaré inequality

$$
\iint_{\Omega} \alpha(x)|u(x, y)|^{2} \leq C \iint_{\Omega} \alpha(x)\left|\partial_{y} u(x, y)\right|^{2} d x d y
$$


for all $u \in H^{1,0}(\Omega), \alpha \in C_{b}^{\infty}\left(\mathbf{R}^{d}\right), \alpha \geq 0$, with a constant $C$ independent of $\alpha$. Indeed, this follows from the same computation as above using the fact that any $u \in H^{1,0}(\Omega)$ can be approximated by a sequence of functions which are $C^{\infty}$ in $\Omega$ and vanish near $y=\eta(x)$.

Proposition 3.3. For every $\psi \in H_{u l}^{\frac{1}{2}}\left(\mathbf{R}^{d}\right)$ the problem

$$
\Delta_{x, y} \Phi=0 \quad \text { in } \Omega,\left.\quad \Phi\right|_{\Sigma}=\psi,\left.\quad \frac{\partial \Phi}{\partial v}\right|_{\Gamma}=0,
$$

has a unique solution $\Phi \in H_{u l}^{1}(\Omega)$ and there exists a function $\mathcal{F}: \mathbf{R}^{+} \rightarrow \mathbf{R}^{+}$independent of $(\psi, \eta)$ such that

$$
\|\Phi\|_{H_{u l}^{1}(\Omega)} \leq \mathcal{F}\left(\|\eta\|_{W^{1, \infty}\left(\mathbf{R}^{d}\right)}\right)\|\psi\|_{H_{u l}^{\frac{1}{2}}\left(\mathbf{R}^{d}\right)} .
$$

Proof. Before giving the proof we have to precise the meaning of the boundary condition $\left.\frac{\partial \Phi}{\partial \nu}\right|_{\Gamma}=0$ since $\Gamma$ is only $C^{0}$. This condition means that

$$
\iint_{\Omega} \nabla_{x, y} \Phi(x, y) \cdot \nabla_{x, y}(\alpha(x) \theta(x, y)) d x d y=0
$$

for every $\theta \in H^{1}(\Omega)$ (the usual Sobolev space) with $\operatorname{supp} \theta \subset\left\{(x, y): \eta_{*}(x) \leq y \leq \eta_{*}(x)+\varepsilon\right\}$ for a small $\varepsilon>0$ and every $\alpha \in C_{0}^{\infty}\left(\mathbf{R}^{d}\right)$.

Notice that if $\eta_{*} \in W^{2, \infty}\left(\mathbf{R}^{d}\right)$ the Green formula (see [20, p. 62]) shows that (3.5) is equivalent to $\left.\frac{\partial \Phi}{\partial v}\right|_{\Gamma}=0$.

Lemma 3.4. We have

$$
\iint_{\Omega} \nabla_{x, y} \Phi(x, y) \cdot \nabla_{x, y}(\alpha(x) \theta(x, y)) d x d y=0
$$

for every $\theta \in H^{1}(\Omega)$ with $\gamma_{0} \theta=0$ and every $\alpha \in C_{0}^{\infty}\left(\mathbf{R}^{d}\right)$.

Proof. If $\theta$ has support in a neighborhood of $\Gamma, V_{\Gamma}=\left\{(x, y): x \in \mathbf{R}^{d}, \eta_{*}(x) \leq y \leq \eta_{*}(x)+\varepsilon\right\}$, this follows from (3.5). Assume that $\theta$ vanishes in a neighborhood of $\Gamma$. Let $\Omega_{0}=\left\{(x, y): \eta_{*}(x)+\frac{\varepsilon}{2}<y<\eta(x)\right\}$ for $\varepsilon>0$ small enough. Then $\theta \in H^{1}\left(\Omega_{0}\right)$ and $\left.\theta\right|_{\partial \Omega_{0}}=0$. Thus $\theta \in H_{0}^{1}\left(\Omega_{0}\right)$. Since $\Omega_{0}$ has a Lipschitz upper boundary there exists a sequence $\theta_{n} \in C_{0}^{\infty}\left(\Omega_{0}\right)$ which converges to $\theta$ in $H^{1}\left(\Omega_{0}\right)$ (see [20, Corollary 1.5.1.6]). Now by the equation we have

$$
0=\left\langle\Delta_{x, y} \Phi, \alpha \theta_{n}\right\rangle=-\iint_{\Omega} \nabla_{x, y} \Phi(x, y) \cdot \nabla_{x, y}\left(\alpha(x) \theta_{n}(x, y)\right) d x d y .
$$

Moreover

$$
\left|\iint_{\Omega} \nabla_{x, y} \Phi(x, y) \cdot \nabla_{x, y}\left(\alpha(x)\left(\theta_{n}-\theta\right)(x, y)\right) d x d y\right| \leq C\|\Phi\|_{H_{u l}^{1}(\Omega)}\left\|\theta_{n}-\theta\right\|_{H^{1}\left(\Omega_{0}\right)} .
$$

Therefore, passing to the limit, we obtain (3.6) for such $\theta$.

Part 1. Uniqueness: Let us denote by $\Phi$ the difference of two solutions in $H_{u l}^{1}(\Omega)$ of (3.4). Then $\gamma_{0} \Phi=0$. Now

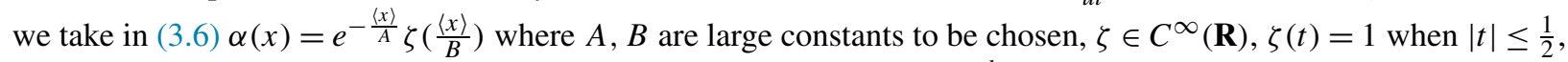
$\operatorname{supp} \zeta \subset\{t \in \mathbf{R}:|t| \leq 1\}, 0 \leq \zeta \leq 1$ and $\theta=\alpha_{1}(x) \Phi$ where $\alpha_{1} \in C_{0}^{\infty}\left(\mathbf{R}^{d}\right)$ is equal to one on the support of $\alpha$. Then $\theta \in H^{1}(\Omega)$ and $\gamma_{0} \theta=0$. We can therefore use Lemma 3.4 and we obtain 


$$
\begin{aligned}
I:= & \iint_{\Omega} \alpha(x)\left|\nabla_{x, y} \Phi(x, y)\right|^{2} d x d y \\
= & \frac{1}{A} \iint_{\Omega}\left(\nabla_{x}\langle x\rangle\right) \alpha(x) \Phi(x, y) \cdot \nabla_{x} \Phi(x, y) d x d y \\
& -\frac{1}{B} \iint_{\Omega} e^{-\frac{\langle x\rangle}{A}} \zeta^{\prime}\left(\frac{\langle x\rangle}{B}\right) \Phi(x, y)\left(\nabla_{x}\langle x\rangle\right) \cdot \nabla_{x} \Phi(x, y) d x d y \\
= & (1)+(2) .
\end{aligned}
$$

By the Cauchy-Schwarz inequality we have

$$
|(1)| \leq \frac{C_{1}}{A}\left(\iint_{\Omega} \alpha(x)\left|\nabla_{x} \Phi(x, y)\right|^{2} d x d y\right)^{\frac{1}{2}}\left(\iint_{\Omega} \alpha(x)|\Phi(x, y)|^{2} d x d y\right)^{\frac{1}{2}} .
$$

Using Lemma 3.1 we deduce that

$$
|(1)| \leq \frac{C_{2}}{A}\left(\iint_{\Omega} \alpha(x)\left|\nabla_{x} \Phi(x, y)\right|^{2} d x d y+\iint_{\Omega} \alpha(x)\left|\partial_{y} \Phi(x, y)\right|^{2} d x d y\right) .
$$

Taking $A$ large enough we see that the term (1) can be absorbed by the left hand side of (3.7). We then fix $A$. It follows that

$$
I \leq \frac{C_{3}}{B} \sum_{q \in \mathbf{Z}^{d}} \sum_{k \in \mathbf{Z}^{d}} \iint_{\Omega} e^{-\frac{\langle x\rangle}{A}}\left|\zeta^{\prime}\left(\frac{\langle x\rangle}{B}\right)\right|\left|\chi_{q}(x) \Phi(x, y)\right|\left|\nabla_{x}\left(\chi_{k}(x) \Phi(x, y)\right)\right| d x d y .
$$

If $|k-q| \geq 2$ we have $\operatorname{supp} \chi_{q} \cap \operatorname{supp} \chi_{k}=\emptyset$. Therefore we have $|k-q| \leq 1$ (essentially $k=q$ ). Moreover in the integral in the right hand side we have $|x-q| \leq 1$ and $\frac{1}{2} \leq \frac{\langle x\rangle}{B} \leq 1$. If $B$ is large enough we have therefore $\frac{B}{3} \leq|q| \leq 3 B$ and $|x| \geq \frac{1}{2}|q|$. It follows that

$$
I \leq \frac{C_{4}}{B} \sum_{\frac{B}{3} \leq|q| \leq 3 B} I_{q}
$$

where

$$
I_{q}=e^{-\frac{\langle q\rangle}{C_{5} A}}\left(\iint_{\Omega}\left|\chi_{q}(x) \Phi(x, y)\right|^{2} d x d y\right)^{\frac{1}{2}}\left(\iint_{\Omega}\left|\nabla_{x}\left(\chi_{q}(x) \Phi(x, y)\right)\right|^{2} d x d y\right)^{\frac{1}{2}}
$$

so using again the Poincaré inequality we obtain

$$
\begin{aligned}
I & \leq \frac{C_{6}}{B} \sum_{\frac{B}{3} \leq|q| \leq 3 B} e^{-\frac{\langle q\rangle}{C_{5} A}} \iint_{\Omega}\left|\nabla_{x, y}\left(\chi_{q}(x) \Phi(x, y)\right)\right|^{2} d x d y \\
& \leq \frac{C_{7}}{B}\left(\sum_{\frac{B}{3} \leq|q| \leq 3 B} e^{-\frac{\langle q\rangle}{C_{5} A}}\right)\|\Phi\|_{H_{u l}^{1}(\Omega)}^{2} .
\end{aligned}
$$

Since the cardinal of the set $\left\{q \in \mathbf{Z}^{d}: \frac{B}{3} \leq|q| \leq 3 B\right\}$ is bounded by $C B^{d}$ we obtain eventually

$$
\iint_{\Omega} e^{-\frac{\langle x\rangle}{A}} \theta\left(\frac{\langle x\rangle}{B}\right)\left|\nabla_{x, y} \Phi(x, y)\right|^{2} d x d y \leq C_{8} B^{d-1} e^{-c \frac{\langle B\rangle}{A}}\|\Phi\|_{H_{u l}^{1}(\Omega)}^{2} .
$$

Letting $B$ go to $+\infty$ and applying Fatou's Lemma we obtain

$$
\iint_{\Omega} e^{-\frac{\langle x\rangle}{A}}\left|\nabla_{x, y} \Phi(x, y)\right|^{2} d x d y=0,
$$

which implies that $\nabla_{x, y} \Phi(x, y)=0$ in $\Omega$ thus $\Phi=0$ since $\left.\Phi\right|_{\Sigma}=0$. 
Part 2: Existence. We first recall the situation when $\psi \in H^{\frac{1}{2}}\left(\mathbf{R}^{d}\right)$. In the following lemma, whose proof is given below, in Section 4.2, we construct a suitable extension of $\psi$ to $\Omega$.

Lemma 3.5. Let $\psi \in H^{\frac{1}{2}}\left(\mathbf{R}^{d}\right)$. One can find $\underline{\psi}$ such that

(1) $\psi \in H^{1}(\Omega)$, supp $\psi \subset\{(x, y): \eta(x)-h \leq y \leq \eta(x)\}$,

(2) $\bar{\psi}_{\mid y=\eta(x)}=\psi(x)$,

(3) $\|\underline{\psi}\|_{H^{1}(\Omega)} \leq \mathcal{F}\left(\|\eta\|_{W^{1, \infty}\left(\mathbf{R}^{d}\right)}\right)\|\psi\|_{H^{\frac{1}{2}}\left(\mathbf{R}^{d}\right)}$.

Then (see [2] for more details) the problem

$$
\Delta_{x, y} u=-\Delta_{x, y} \underline{\psi} \quad \text { in } \Omega,\left.\quad u\right|_{\Sigma}=0,\left.\quad \frac{\partial u}{\partial v}\right|_{\Gamma}=0
$$

has a unique solution $u \in H^{1,0}(\Omega)$. This solution, which is the variational one, is characterized by

$$
\iint_{\Omega} \nabla_{x, y} u(x, y) \cdot \nabla_{x, y} \theta(x, y) d x d y=-\iint_{\Omega} \nabla_{x, y} \underline{\psi}(x, y) \cdot \nabla_{x, y} \theta(x, y) d x d y
$$

for every $\theta \in H^{1,0}(\Omega)$. It satisfies

$$
\left\|\nabla_{x, y} u\right\|_{L^{2}(\Omega)} \leq C\|\psi\|_{H^{\frac{1}{2}}\left(\mathbf{R}^{d}\right)} .
$$

Then $\Phi=u+\underline{\psi}$ solves the problem (3.4).

Let us consider now the case where $\psi \in H_{u l}^{\frac{1}{2}}\left(\mathbf{R}^{d}\right)$. If $q \in \mathbf{Z}^{d}$ and $\chi_{q}$ is defined in (2.3) we set

$$
\psi_{q}=\chi_{q} \psi \in H^{\frac{1}{2}}\left(\mathbf{R}^{d}\right) .
$$

By Lemma 3.5 one can find $\underline{\psi}_{q} \in H^{1}(\Omega)$ such that $\left.\underline{\psi}_{q}\right|_{y=\eta(x)}=\psi_{q}(x)$ and

(i) $\operatorname{supp} \underline{\psi}_{q} \subset\{(x, y):|x-q| \leq 2, \eta(x)-h \leq y \leq \eta(x)\}$

(ii) $\quad\left\|\underline{\psi}_{q}\right\|_{H^{1}(\Omega)} \leq \mathcal{F}\left(\|\eta\|_{W^{1, \infty}\left(\mathbf{R}^{d}\right)}\right)\left\|\psi_{q}\right\|_{H^{\frac{1}{2}}\left(\mathbf{R}^{d}\right)}$.

To achieve $(i)$ we multiply the function constructed in the lemma by $\tilde{\chi}_{q}(x)$, where supp $\tilde{\chi}$ is contained in $\{x:|x| \leq 2\}$ and $\tilde{\chi}=1$ on the support of $\chi$.

Let $u_{q}$ be the variational solution, described above, of the equation $\Delta_{x, y} u_{q}=-\Delta_{x, y} \psi_{q}$. Our aim is to prove that the series $\sum_{q \in \mathbf{Z}^{d}} u_{q}$ is convergent in the space $H_{u l}^{1,0}(\Omega)$. This will be a consequence of the following lemma.

Lemma 3.6. There exist $\delta>0$ and $\mathcal{F}: \mathbf{R}^{+} \rightarrow \mathbf{R}^{+}$non-decreasing such that for all $q \in \mathbf{Z}^{d}$ we have

$$
\left\|e^{\delta\langle x-q\rangle} \nabla_{x, y} u_{q}\right\|_{L^{2}(\Omega)} \leq \mathcal{F}\left(\|\eta\|_{W^{1, \infty}\left(\mathbf{R}^{d}\right)}\right)\left\|\psi_{q}\right\|_{H^{\frac{1}{2}}\left(\mathbf{R}^{d}\right)} .
$$

Assuming that this lemma has been proved, one can write

$$
\begin{aligned}
\left\|\chi_{k} \nabla_{x, y} u_{q}\right\|_{L^{2}(\Omega)} & =\left\|\chi_{k} e^{-\delta\langle x-q\rangle} e^{\delta\langle x-q\rangle} \nabla_{x, y} u_{q}\right\|_{L^{2}(\Omega)} \\
& \leq C e^{-\delta\langle k-q\rangle} \mathcal{F}\left(\|\eta\|_{W^{1, \infty}}\right)\left\|\psi_{q}\right\|_{H^{\frac{1}{2}}} \\
& \leq C^{\prime} e^{-\delta\langle k-q\rangle} \mathcal{F}\left(\|\eta\|_{W^{1, \infty}}\right)\|\psi\|_{H_{u l}^{\frac{1}{2}}}
\end{aligned}
$$

Let us set $S^{Q}=\sum_{|q| \leq Q} u_{q}$. First of all $\left(S^{Q}\right)$ converges to $u=\sum_{q \in \mathbf{Z}^{d}} u_{q}$ in $\mathcal{D}^{\prime}(\Omega)$. Indeed if $\varphi \in C_{0}^{\infty}(\Omega)$ there exists a finite set $A \subset \mathbf{Z}^{d}$ such that $\varphi=\sum_{k \in A} \chi_{k} \varphi$. Then using Lemma 3.1 and (3.10) we can write 


$$
\begin{aligned}
\left|\left\langle u_{q}, \varphi\right\rangle\right| & \leq \sum_{k \in A}\left|\left\langle\chi_{k} u_{q}, \varphi\right\rangle\right| \leq C \sum_{k \in A}\left\|\chi_{k} \partial_{y} u_{q}\right\|_{L^{2}(\Omega)}\|\varphi\|_{L^{2}(\Omega)} \\
& \leq C e^{-\delta\langle q\rangle} \mathcal{F}\left(\|\eta\|_{W^{1, \infty}}\right)\|\psi\|_{H_{u l}^{\frac{1}{2}}\|\varphi\|_{L^{2}(\Omega)}}
\end{aligned}
$$

for large $|q|$.

On the other hand (3.10) shows that for fixed $k$ the series $\sum_{q \in \mathbf{Z}^{d}} \chi_{k} u_{q}$ is absolutely convergent in $H_{u l}^{1,0}(\Omega)$. Therefore $\left(\chi_{k} S^{Q}\right)$ converges to $\left(\chi_{k} u\right)$ in $H_{u l}^{1,0}(\Omega)$ and we can write using (3.10),

$$
\begin{aligned}
\left\|\chi_{k} \nabla_{x, y} u\right\|_{L^{2}(\Omega)} & =\lim _{Q \rightarrow+\infty}\left\|\chi_{k} \nabla_{x, y} S^{Q}\right\|_{L^{2}(\Omega)} \\
& \leq \sum_{q \in \mathbf{Z}^{d}} e^{-\delta\langle k-q\rangle} \mathcal{F}\left(\|\eta\|_{W^{1, \infty}}\right)\|\psi\|_{H_{u l}^{\frac{1}{2}}} .
\end{aligned}
$$

Therefore $u \in H_{u l}^{1,0}(\Omega)$ and

$$
\left\|\nabla_{x, y} u\right\|_{L_{u l}^{2}(\Omega)} \leq \mathcal{F}\left(\|\eta\|_{W^{1, \infty}}\right)\|\psi\|_{H_{u l}^{\frac{1}{2}}} .
$$

Finally $\Phi=u+\underline{\psi}$ solves the problem (3.4) and we have

$$
\|\Phi\|_{H_{u l}^{1}(\Omega)} \leq \mathcal{F}\left(\|\eta\|_{W^{1, \infty}}\right)\|\psi\|_{H_{u l}^{\frac{1}{2}}}
$$

which completes the proof of Proposition 3.3 assuming Lemma 3.6.

Proof of Lemma 3.6. We set

$$
w_{\varepsilon}(x)=\frac{\langle x-q\rangle}{1+\varepsilon\langle x-q\rangle} .
$$

Let $u_{q}$ be the variational solution in $H^{1,0}(\Omega)$ of $\Delta u_{q}=-\Delta \underline{\psi}_{q}$. According to the variational formulation (3.8), with $\theta=e^{2 \delta w_{\varepsilon}(x)} u_{q}$, we have

$$
\iint_{\Omega} \nabla_{x, y} u_{q} \cdot \nabla_{x, y}\left(e^{2 \delta w_{\varepsilon}(x)} u_{q}\right) d x d y=-\iint_{\Omega} \nabla_{x, y} \underline{\psi}_{q} \cdot \nabla_{x, y}\left(e^{2 \delta w_{\varepsilon}(x)} u_{q}\right) d x d y .
$$

Therefore

$$
\begin{aligned}
\iint_{\Omega} e^{2 \delta w_{\varepsilon}(x)} \nabla_{x, y} u_{q} \cdot \nabla_{x, y} u_{q} d x d y= & -\iint_{\Omega} e^{2 \delta w_{\varepsilon}(x)} \nabla_{x, y} \underline{\psi}_{q} \cdot \nabla_{x, y} u_{q} d x d y \\
& -2 \delta \iint_{\Omega} e^{2 \delta w_{\varepsilon}(x)} u_{q} \nabla_{x} \underline{\psi}_{q} \cdot \nabla_{x} w_{\varepsilon} d x d y \\
& -2 \delta \iint_{\Omega} e^{2 \delta w_{\varepsilon}(x)} u_{q} \nabla_{x} u_{q} \cdot \nabla_{x} w_{\varepsilon} d x d y .
\end{aligned}
$$

Now $\nabla_{x} w_{\varepsilon}$ is uniformly bounded in $L^{\infty}$ with respect to $\varepsilon$ and $x$ and, on the support of $\psi_{q}$, we have $e^{\delta w_{\varepsilon}(x)} \leq e^{C \delta}$. Consequently, using the Cauchy-Schwarz inequality, the inequality (3.3) with $\alpha=e^{2 \delta w_{\varepsilon}(x)}$ and taking $\delta$ small enough we obtain

$$
\iint_{\Omega} e^{2 \delta w_{\varepsilon}(x)}\left|\nabla_{x, y} u_{q}\right|^{2} d x d y \leq C\left\|\underline{\psi_{q}}\right\|_{H^{1}(\Omega)}^{2} .
$$

We deduce when $\varepsilon$ goes to 0 , using the Fatou Lemma, that

$$
\left\|e^{\delta\langle x-q\rangle} \nabla_{x, y} u_{q}\right\|_{L^{2}(\Omega)} \leq C\left\|\underline{\psi}_{q}\right\|_{H^{1}(\Omega)} \leq \mathcal{F}\left(\|\eta\|_{W^{1, \infty}\left(\mathbf{R}^{d}\right)}\right)\left\|\psi_{q}\right\|_{H^{\frac{1}{2}\left(\mathbf{R}^{d}\right)}} .
$$

This completes the proof. 


\subsection{Straightening the boundary}

Before studying more precisely the properties of the Dirichlet-Neumann operator, we first straighten the boundaries of

$$
\Omega_{h}=\left\{(x, y) \in \mathbf{R}^{d+1}: \eta(x)-h<y<\eta(x)\right\} .
$$

Lemma 3.7. There is an absolute constant $C>0$ such that if we take $\delta>0$ so small that

$$
\delta\|\eta\|_{W^{1, \infty}\left(\mathbf{R}^{d}\right)} \leq \frac{h}{2 C}
$$

then the map $(x, z) \mapsto(x, \rho(x, z))$ where

$$
\rho(x, z)=(1+z) e^{\delta z\left\langle D_{x}\right\rangle} \eta(x)-z\left\{e^{-\delta(1+z)\left\langle D_{x}\right\rangle} \eta(x)-h\right\}
$$

is a diffeomorphism from $\widetilde{\Omega}=\left\{(x, z): x \in \mathbf{R}^{d},-1<z<0\right\}$ to $\Omega_{h}$.

Proof. First of all we have $\rho(x, 0)=\eta(x), \rho(x,-1)=\eta(x)-h$. Moreover we have $\partial_{z} \rho \geq \frac{h}{2}$. Indeed we have

$$
\begin{aligned}
\partial_{z} \rho= & \eta+\left(e^{\delta z\left\langle D_{x}\right\rangle} \eta-\eta\right)+(1+z) \delta e^{\delta z\left\langle D_{x}\right\rangle}\left\langle D_{x}\right\rangle \eta \\
& -\left\{\eta-h+\left(e^{-\delta(1+z)\left\langle D_{x}\right\rangle} \eta-\eta\right)\right\}+z \delta e^{-\delta(1+z)\left\langle D_{x}\right\rangle}\left\langle D_{x}\right\rangle \eta .
\end{aligned}
$$

Now for any $\lambda<0$ the symbol $a(\xi)=e^{\lambda\langle\xi\rangle}$ satisfies the estimate $\left|\partial_{\xi}^{\alpha} a(\xi)\right| \leq C_{\alpha}\langle\xi\rangle^{-|\alpha|}$ where $C_{\alpha}$ is independent of $\lambda$. Therefore its Fourier transform is an $L^{1}\left(\mathbf{R}^{d}\right)$ function whose norm is uniformly bounded. This implies that $\|a(D) f\|_{L^{\infty}\left(\mathbf{R}^{d}\right)} \leq K\|f\|_{L^{\infty}\left(\mathbf{R}^{d}\right)}$ with $K$ independent of $\lambda$. Since $e^{\delta \lambda\left\langle D_{x}\right\rangle} \eta-\eta=\delta \lambda \int_{0}^{1} e^{\delta t \lambda\left\langle D_{x}\right\rangle}\left\langle D_{x}\right\rangle \eta d t$ we can write

$$
\begin{aligned}
& \left\|e^{\delta z\left\langle D_{x}\right\rangle} \eta-\eta\right\|_{L^{\infty}\left(\mathbf{R}^{d}\right)}+\delta\left\|e^{\delta z\left\langle D_{x}\right\rangle}\left\langle D_{x}\right\rangle \eta\right\|_{L^{\infty}\left(\mathbf{R}^{d}\right)}+\left\|e^{-\delta(1+z)\left\langle D_{x}\right\rangle} \eta-\eta\right\|_{L^{\infty}\left(\mathbf{R}^{d}\right)} \\
& +\delta\left\|e^{-\delta(1+z)\left\langle D_{x}\right\rangle}\left\langle D_{x}\right\rangle \eta\right\|_{L^{\infty}\left(\mathbf{R}^{d}\right)} \leq C \delta\|\eta\|_{W^{1, \infty}\left(\mathbf{R}^{d}\right)} \leq \frac{h}{2} .
\end{aligned}
$$

This completes the proof.

From the above computation we deduce that

$$
\partial_{z} \rho(x, z) \geq \frac{h}{2} \quad \text { and } \quad\left\|\nabla_{x, z} \rho\right\|_{L^{\infty}\left(\mathbf{R}^{d}\right)} \leq C\|\eta\|_{W^{1, \infty}\left(\mathbf{R}^{d}\right)} .
$$

We shall denote by $\kappa$ the inverse of $\rho$,

$$
\rho(x, z)=y \quad \Longleftrightarrow \quad z=\kappa(x, y) .
$$

If we set

$$
\tilde{f}(x, z)=f(x, \rho(x, z))
$$

we have

$$
\left\{\begin{array}{l}
\frac{\partial f}{\partial y}(x, \rho(x, z))=\frac{1}{\partial_{z} \rho} \partial_{z} \tilde{f}(x, z)=: \Lambda_{1} \tilde{f}(x, z) \\
\nabla_{x} f(x, \rho(x, z))=\left(\nabla_{x} \tilde{f}-\frac{\nabla_{x} \rho}{\partial_{z} \rho} \partial_{z} \tilde{f}\right)(x, z)=: \Lambda_{2} \tilde{f}(x, z) .
\end{array}\right.
$$

We introduce the space

$$
\mathcal{H}_{u l}^{1}(\widetilde{\Omega})=\left\{\tilde{u} \in L_{u l}^{2}(\widetilde{\Omega}): \Lambda_{j} \tilde{u} \in L_{u l}^{2}(\widetilde{\Omega}), j=1,2\right\},
$$

endowed with the norm 


$$
\|\tilde{u}\|_{\mathcal{H}_{u l}^{1}(\widetilde{\Omega})}=\sup _{q \in \mathbf{Z}^{d}}\left\|\chi_{q} \tilde{u}\right\|_{L^{2}(\widetilde{\Omega})}+\sum_{j=1}^{2} \sup _{q \in \mathbf{Z}^{d}}\left\|\chi_{q} \Lambda_{j} \tilde{u}\right\|_{L^{2}(\widetilde{\Omega})} .
$$

Then according to Lemma 7.12 we see that the elements of $\mathcal{H}_{u l}^{1}(\widetilde{\Omega})$ have a trace on $z=0$ belonging to the space $\mathcal{H}_{u l}^{\frac{1}{2}}\left(\mathbf{R}^{d}\right)$. Then we introduce the subspace $\mathcal{H}_{u l}^{1,0}(\widetilde{\Omega}) \subset \mathcal{H}_{u l}^{1}(\widetilde{\Omega})$ defined as follows

$$
\mathcal{H}_{u l}^{1,0}(\widetilde{\Omega})=\left\{\tilde{u} \in \mathcal{H}_{u l}^{1}(\widetilde{\Omega}):\left.\tilde{u}\right|_{z=0}=0\right\} .
$$

It follows that we have

$$
u \in H_{u l}^{1}(\Omega) \quad \Leftrightarrow \quad \tilde{u} \in \mathcal{H}_{u l}^{1}(\widetilde{\Omega}), \quad u \in H_{u l}^{1,0}(\Omega) \quad \Leftrightarrow \quad \tilde{u} \in \mathcal{H}_{u l}^{1,0}(\widetilde{\Omega}) .
$$

\subsection{Definition of the Dirichlet-Neumann operator}

We can now define the Dirichlet-Neumann operator. Formally we set for $\psi \in H_{u l}^{\frac{1}{2}}\left(\mathbf{R}^{d}\right)$

$$
\begin{aligned}
G(\eta) \psi(x) & =\left.\left(1+\left|\nabla_{x} \eta\right|^{2}\right)^{\frac{1}{2}} \frac{\partial \Phi}{\partial n}\right|_{\Sigma}=\left.\left(\frac{\partial \Phi}{\partial y}-\nabla_{x} \eta \cdot \nabla_{x} \Phi\right)\right|_{\Sigma} \\
& =\left.\left(\Lambda_{1} \widetilde{\Phi}-\nabla_{x} \eta \cdot \Lambda_{2} \widetilde{\Phi}\right)\right|_{z=0}=\left.\left(\Lambda_{1} \widetilde{\Phi}-\nabla_{x} \rho \cdot \Lambda_{2} \widetilde{\Phi}\right)\right|_{z=0}
\end{aligned}
$$

where $\Phi$ is the solution described in Lemma 3.3 and $\Lambda_{j}$ is as defined by (3.17). Our aim is to prove the following theorem.

Theorem 3.8. Let $d \geq 1$ and $\eta \in W^{1, \infty}\left(\mathbf{R}^{d}\right)$. Then the Dirichlet-Neumann operator is well defined on $H_{u l}^{\frac{1}{2}}\left(\mathbf{R}^{d}\right)$ by (3.18). Moreover there exists a non-decreasing function $\mathcal{F}: \mathbf{R}^{+} \rightarrow \mathbf{R}^{+}$such that for all $\eta \in W^{1, \infty}\left(\mathbf{R}^{d}\right)$

$$
\|G(\eta) \psi\|_{H_{u l}^{-\frac{1}{2}}\left(\mathbf{R}^{d}\right)} \leq \mathcal{F}\left(\|\eta\|_{W^{1, \infty}\left(\mathbf{R}^{d}\right)}\right)\|\psi\|_{H_{u l}^{\frac{1}{2}}\left(\mathbf{R}^{d}\right)} .
$$

Proof. Set $U=\Lambda_{1} \widetilde{\Phi}-\nabla_{x} \rho \cdot \Lambda_{2} \widetilde{\Phi}$ and $J=(-1,0)$. We shall prove that for all $q \in \mathbf{Z}^{d}$,

$$
\begin{aligned}
& \left\|\chi_{q} U\right\|_{L^{2}\left(J, L^{2}\right)} \leq \mathcal{F}\left(\|\eta\|_{W^{1, \infty}}\right)\|\psi\|_{H_{u l}^{\frac{1}{2}},} \\
& \left\|\chi_{q} \partial_{z} U\right\|_{L^{2}\left(J, H^{-1}\right)} \leq \mathcal{F}\left(\|\eta\|_{W^{1, \infty}}\right)\|\psi\|_{H_{u l}^{\frac{1}{2}}},
\end{aligned}
$$

where $\mathcal{F}: \mathbf{R}^{+} \rightarrow \mathbf{R}^{+}$is independent of $q$ and $\eta$. Then Theorem 3.8 will follow from (3.19), (3.20) and Lemma 7.12. Recall that $\widetilde{\Phi}=\tilde{u}+\tilde{\psi}$. Now the estimate (3.19) follows from (3.11), (3.17) and Corollary 7.11 with $\sigma=0$ and $m=1$. To prove (3.20) we observe that

$$
\partial_{z} U=-\nabla_{x} \cdot\left(\left(\partial_{z} \rho\right) \Lambda_{2} \widetilde{\Phi}\right) .
$$

Indeed we have

$$
\begin{aligned}
\partial_{z} U & =\partial_{z} \Lambda_{1} \widetilde{\Phi}-\nabla_{x} \partial_{z} \rho \cdot \Lambda_{2} \widetilde{\Phi}-\nabla_{x} \rho \cdot \partial_{z} \Lambda_{2} \widetilde{\Phi} \\
& =\left(\partial_{z} \rho\right) \Lambda_{1}^{2} \widetilde{\Phi}-\nabla_{x} \partial_{z} \rho \cdot \Lambda_{2} \widetilde{\Phi}+\left(\partial_{z} \rho\right)\left(\Lambda_{2}-\nabla_{x}\right) \cdot \Lambda_{2} \widetilde{\Phi} \\
& =\left(\partial_{z} \rho\right)\left(\Lambda_{1}^{2}+\Lambda_{2}^{2}\right) \widetilde{\Phi}-\nabla_{x} \cdot\left(\left(\partial_{z} \rho\right) \Lambda_{2} \widetilde{\Phi}\right)
\end{aligned}
$$

so (3.21) follows from the fact that $\left(\Lambda_{1}^{2}+\Lambda_{2}^{2}\right) \widetilde{\Phi}=0$. Then (3.20) follows from the estimates used to bound (3.19) and the Poincaré inequality (3.3). The proof of Theorem 3.8 is complete.

We state now a consequence of the previous estimates which will be used in the sequel. Notice first that the equation $\left(\Lambda_{1}^{2}+\Lambda_{2}^{2}\right) \widetilde{\Phi}=0$ is equivalent to the equation

$$
\left(\partial_{z}^{2}+\alpha \Delta_{x}+\beta \cdot \nabla_{x} \partial_{z}-\gamma \partial_{z}\right) \widetilde{\Phi}=0,
$$


where

$$
\alpha:=\frac{\left(\partial_{z} \rho\right)^{2}}{1+\left|\nabla_{x} \rho\right|^{2}}, \quad \beta:=-2 \frac{\partial_{z} \rho \nabla_{x} \rho}{1+\left|\nabla_{x} \rho\right|^{2}}, \quad \gamma:=\frac{1}{\partial_{z} \rho}\left(\partial_{z}^{2} \rho+\alpha \Delta_{x} \rho+\beta \cdot \nabla_{x} \partial_{z} \rho\right) .
$$

Corollary 3.9. Let $s>1+\frac{d}{2}$ and $J=(-1,0)$. There exists a non-decreasing function $\mathcal{F}: \mathbf{R}^{+} \rightarrow \mathbf{R}^{+}$such that

$$
\left\|\nabla_{x, z} \widetilde{\Phi}\right\|_{X_{u l}^{-\frac{1}{2}}(J)} \leq \mathcal{F}\left(\|\eta\|_{H_{u l}^{s+\frac{1}{2}}\left(\mathbf{R}^{d}\right)}\right)\|\psi\|_{H_{u l}^{\frac{1}{2}}\left(\mathbf{R}^{d}\right)}
$$

where the spaces $X_{u l}^{\sigma}(J), \sigma \in \mathbf{R}$, are defined in Definition 7.4.

Proof. Recall that $\widetilde{\Phi}=\tilde{\psi}+\tilde{u}$. First of all the estimate

$$
\left\|\nabla_{x, z} \underline{\tilde{\psi}}_{X_{u l}^{-\frac{1}{2}}(J)} \leq C\right\| \psi \|_{H_{u l}^{\frac{1}{2}}}
$$

follows from Corollary 7.11 with $\delta=1, m=0,1$ and $\sigma=0$.

On the other hand we notice that $\partial_{z}=\left(\partial_{z} \rho\right) \Lambda_{1}$ and $\nabla_{x}=\Lambda_{2}+\left(\nabla_{x} \rho\right) \Lambda_{1}$. Let $\tilde{\chi} \in C_{0}^{\infty}\left(\mathbf{R}^{d}\right), \tilde{\chi}=1$ on the support of $\chi$. Since $s>1+\frac{d}{2}$, using Corollary 7.11 with $\sigma=s$ we can write

$$
\begin{aligned}
\left\|\tilde{\chi}_{k} \nabla_{x, z} \rho\right\|_{L^{\infty}\left(J \times \mathbf{R}^{d}\right)} & \leq C\left\|\tilde{\chi}_{k} \nabla_{x, z} \rho\right\|_{L^{\infty}\left(J, H^{\left.s-\frac{1}{2}\right)}\right.} \leq C^{\prime}\left\|\nabla_{x, z} \rho\right\|_{L^{\infty}\left(J, H^{s-\frac{1}{2}}\right)_{u l}} \\
& \leq C^{\prime \prime}\left(1+\|\eta\|_{\left.H_{u l}^{s+\frac{1}{2}}\right) .}\right.
\end{aligned}
$$

It follows from (3.11) that $\left\|\nabla_{x, z} \tilde{u}\right\|_{L^{2}\left(J, L^{2}\right)_{u l}} \leq \mathcal{F}\left(\|\eta\|_{H_{u l}^{s+\frac{1}{2}}}\right)\|\psi\|_{H_{u l}^{\frac{1}{2}}}$, which implies that

$$
\left\|\nabla_{x, z} \widetilde{\Phi}\right\|_{L^{2}\left(J, L^{2}\right)_{u l}} \leq \mathcal{F}\left(\|\eta\|_{H_{u l}^{s+\frac{1}{2}}}\right)\|\psi\|_{H_{u l}^{\frac{1}{2}}} .
$$

Now using Lemma 7.12 we have

$$
\left\|\chi_{k} \nabla_{x} \widetilde{\Phi}\right\|_{L^{\infty}\left(J, H^{\left.-\frac{1}{2}\right)}\right.} \leq C\left(\left\|\chi_{k} \nabla_{x} \widetilde{\Phi}\right\|_{L^{2}\left(J, L^{2}\right)}+\left\|\chi_{k} \partial_{z} \nabla_{x} \widetilde{\Phi}\right\|_{L^{2}\left(J, H^{-1}\right)}\right) .
$$

The first term in the right hand side is estimated using (3.25). For the second term using (3.25) we have

$$
\begin{aligned}
\left\|\chi_{k} \partial_{z} \nabla_{x} \widetilde{\Phi}\right\|_{L^{2}\left(J, H^{-1}\right)} & \leq\left\|\left(\nabla_{x} \chi_{k}\right) \partial_{z} \widetilde{\Phi}\right\|_{L^{2}\left(J, H^{-1}\right)}+\left\|\chi_{k} \partial_{z} \widetilde{\Phi}\right\|_{L^{2}\left(J, L^{2}\right)} \\
& \leq C\left\|\partial_{z} \widetilde{\Phi}\right\|_{L^{2}\left(J, L^{2}\right)_{u l}} \leq \mathcal{F}\left(\|\eta\|_{H_{u l}}^{s+\frac{1}{2}}\right)\|\psi\|_{H_{u l}^{\frac{1}{2}}}
\end{aligned}
$$

Therefore

$$
\left\|\nabla_{x} \widetilde{\Phi}\right\|_{L^{\infty}\left(J, H^{-\frac{1}{2}}\right)_{u l}} \leq \mathcal{F}\left(\|\eta\|_{H_{u l}^{s+\frac{1}{2}}}\right)\|\psi\|_{H_{u l}^{\frac{1}{2}}} .
$$

Eventually

$$
\left\|\chi_{k} \partial_{z} \widetilde{\Phi}\right\|_{L^{\infty}\left(J, H^{\left.-\frac{1}{2}\right)}\right.} \leq C\left(\left\|\chi_{k} \partial_{z} \widetilde{\Phi}\right\|_{L^{2}\left(J, L^{2}\right)}+\left\|\chi_{k} \partial_{z}^{2} \widetilde{\Phi}\right\|_{L^{2}\left(J, H^{-1}\right)}\right) .
$$

The first term in the right hand side is estimated using (3.25). For the second term using (3.22) we have

$$
\left\{\begin{array}{l}
\left\|\chi_{k} \partial_{z}^{2} \widetilde{\Phi}\right\|_{L^{2}\left(J, H^{-1}\right)} \leq A_{1}+A_{2}+A_{3}, \\
A_{1}=\left\|\chi_{k} \alpha \Delta_{x} \widetilde{\Phi}\right\|_{L^{2}\left(J, H^{-1}\right)}, \\
A_{2}=\left\|\chi_{k} \beta \partial_{z} \nabla_{x} \widetilde{\Phi}\right\|_{L^{2}\left(J, H^{-1}\right)}, \\
A_{3}=\left\|\chi_{k} \gamma \partial_{z} \widetilde{\Phi}\right\|_{L^{2}\left(J, H^{-1}\right)} .
\end{array}\right.
$$

Now using (7.5), (3.25) and (3.26) we obtain 


$$
\begin{aligned}
& A_{1} \leq\|\alpha\|_{L^{\infty}\left(J, H^{s-\frac{1}{2}}\right)_{u l}}\left\|\Delta_{x} \widetilde{\Phi}\right\|_{L^{2}\left(J, H^{-1}\right)_{u l}} \leq \mathcal{F}\left(\|\eta\|_{H_{u l}^{s+\frac{1}{2}}}\right)\|\psi\|_{H_{u l}^{\frac{1}{2}}}, \\
& A_{2} \leq\|\beta\|_{L^{\infty}\left(J, H^{s-\frac{1}{2}}\right)_{u l}}\left\|\partial_{z} \nabla_{x} \widetilde{\Phi}\right\|_{L^{2}\left(J, H^{-1}\right)_{u l}} \leq \mathcal{F}\left(\|\eta\|_{H_{u l}^{s+\frac{1}{2}}}\right)\|\psi\|_{H_{u l}^{\frac{1}{2}}}, \\
& A_{3} \leq\|\gamma\|_{L^{\infty}\left(J, H^{s-\frac{3}{2}}\right)_{u l}}\left\|\partial_{z} \widetilde{\Phi}\right\|_{L^{2}\left(J, L^{2}\right)_{u l}} \leq \mathcal{F}\left(\|\eta\|_{\left.H_{u l}^{s+\frac{1}{2}}\right)}\|\psi\|_{H_{u l}^{\frac{1}{2}}} .\right.
\end{aligned}
$$

Therefore using (3.28) we obtain

$$
\left\|\partial_{z} \widetilde{\Phi}\right\|_{L^{\infty}\left(J, H^{-\frac{1}{2}}\right)_{u l}} \leq \mathcal{F}\left(\|\eta\|_{H_{u l}^{s+\frac{1}{2}}}\right)\|\psi\|_{H_{u l}^{\frac{1}{2}}}
$$

which completes the proof of Corollary 3.9.

\subsection{Higher estimates for the Dirichlet-Neumann operator}

In this section we prove the following results.

Theorem 3.10. Let $d \geq 1$ and $s_{0}>1+\frac{d}{2}$.

Case 1. There exists $\mathcal{F}: \mathbf{R}^{+} \rightarrow \mathbf{R}^{+}$non-decreasing such that for $-\frac{1}{2} \leq \sigma \leq s_{0}-1$, every $\eta \in H_{u l}^{s_{0}+\frac{1}{2}}\left(\mathbf{R}^{d}\right)$ satisfying (3.2) and every $\psi \in H_{u l}^{\sigma+1}\left(\mathbf{R}^{d}\right)$ we have

$$
\|G(\eta) \psi\|_{H_{u l}^{\sigma}} \leq \mathcal{F}\left(\|\eta\|_{H_{u l}^{s_{0}+\frac{1}{2}}}\right)\|\psi\|_{H_{u l}^{\sigma+1}} .
$$

Case 2. For every $s \geq s_{0}$, there exists $\mathcal{F}: \mathbf{R}^{+} \rightarrow \mathbf{R}^{+}$non-decreasing such that for every $\eta \in H_{u l}^{s+\frac{1}{2}}\left(\mathbf{R}^{d}\right)$ satisfying (3.2), every $s_{0}-1 \leq \sigma \leq s-\frac{1}{2}$ and every $\psi \in H_{u l}^{\sigma+1}\left(\mathbf{R}^{d}\right)$ we have

$$
\|G(\eta) \psi\|_{H_{u l}^{\sigma}} \leq \mathcal{F}\left(\|(\eta, \psi)\|_{H_{u l}^{s_{0}+\frac{1}{2}} \times H_{u l}^{s_{0}}}\right)\left\{\|\eta\|_{H_{u l}^{s+\frac{1}{2}}}+\|\psi\|_{H_{u l}^{\sigma+1}}+1\right\} .
$$

We set

$$
R(\eta) \psi:=G(\eta) \psi-T_{\lambda} \psi
$$

where $T_{\lambda}$ is the paradifferential operator (see Section 7.5) with symbol

$$
\lambda=\left(\left(1+\left|\nabla_{x} \eta\right|^{2}\right)|\xi|^{2}-\left(\nabla_{x} \eta \cdot \xi\right)^{2}\right)^{\frac{1}{2}} .
$$

Theorem 3.11. Let $d \geq 1$ and $s_{0}>1+\frac{d}{2}$.

Case 1. There exists $\mathcal{F}: \mathbf{R}^{+} \rightarrow \mathbf{R}^{+}$non-decreasing such that for $0 \leq t \leq s_{0}-\frac{1}{2}, \eta \in H_{u l}^{s_{0}+\frac{1}{2}}\left(\mathbf{R}^{d}\right)$ satisfying (3.2) we have

$$
\|R(\eta) \psi\|_{H_{u l}^{t}} \leq \mathcal{F}\left(\|\eta\|_{H_{u l} s_{0}+\frac{1}{2}}\right)\|\psi\|_{H_{u l} t+\frac{1}{2}}
$$

for every $\psi \in H_{u l}^{t+\frac{1}{2}}\left(\mathbf{R}^{d}\right)$.

Case 2. For all $s \geq s_{0}$ there exists $\mathcal{F}: \mathbf{R}^{+} \rightarrow \mathbf{R}^{+}$non-decreasing such that for every $\eta \in H_{u l}^{s+\frac{1}{2}}\left(\mathbf{R}^{d}\right)$ satisfying (3.2), every $s_{0}-\frac{1}{2} \leq t \leq s-\frac{1}{2}$ and every $\psi \in H_{u l}^{t+\frac{1}{2}}\left(\mathbf{R}^{d}\right)$ we have

$$
\|R(\eta) \psi\|_{H_{u l}^{t}} \leq \mathcal{F}\left(\|(\eta, \psi)\|_{H_{u l}^{s_{0}+\frac{1}{2}} \times H_{u l}^{s_{0}}}\right)\left\{\|\eta\|_{H_{u l}^{s+\frac{1}{2}}}+\|\psi\|_{H_{u l}^{t+\frac{1}{2}}}+1\right\} .
$$

The main step in the proof of the above theorems is the following elliptic regularity result. 
Theorem 3.12. Let $d \geq 1, J=(-1,0), s_{0}>1+\frac{d}{2}$. Let $\tilde{v}$ be a solution of the problem

$$
\left\{\begin{array}{l}
\left(\partial_{z}^{2}+\alpha \Delta_{x}+\beta \cdot \nabla_{x} \partial_{z}-\gamma \partial_{z}\right) \tilde{v}=F \quad \text { in } \mathbf{R}^{d} \times J, \\
\left.\tilde{v}\right|_{z=0}=\psi .
\end{array}\right.
$$
and

Case 1. For $-\frac{1}{2} \leq \sigma \leq s_{0}-1$ let $\eta \in H_{u l}^{s_{0}+\frac{1}{2}}\left(\mathbf{R}^{d}\right)$ satisfying (3.2), $\psi \in H_{u l}^{\sigma+1}\left(\mathbf{R}^{d}\right), F \in Y_{u l}^{\sigma}(J)$ (see Definition 7.4)

$$
\left\|\nabla_{x, z} \tilde{v}\right\|_{X_{u l}^{-\frac{1}{2}}(J)}<+\infty
$$

Then for every $z_{0} \in(-1,0)$ one has $\nabla_{x, z} \tilde{v} \in X_{u l}^{\sigma}\left(z_{0}, 0\right)$ and one can find $\mathcal{F}: \mathbf{R}^{+} \rightarrow \mathbf{R}^{+}$non-decreasing, depending only on $\left(s_{0}, d\right)$ such that

$$
\left\|\nabla_{x, z} \tilde{v}\right\|_{X_{u l}^{\sigma}\left(z_{0}, 0\right)} \leq \mathcal{F}\left(\|\eta\|_{H_{u l}^{s_{0}+\frac{1}{2}}}\right)\left\{\|\psi\|_{H_{u l}^{\sigma+1}}+\|F\|_{Y_{u l}^{\sigma}(J)}+\left\|\nabla_{x, z} \tilde{v}\right\|_{X_{u l}^{-\frac{1}{2}(J)}}\right\} .
$$

Case 2. For $s \geq s_{0}$, and $s_{0}-1 \leq \sigma \leq s-\frac{1}{2}$ let $\eta \in H_{u l}^{s+\frac{1}{2}}\left(\mathbf{R}^{d}\right)$ satisfying (3.2), $\psi \in H_{u l}^{\sigma+1}\left(\mathbf{R}^{d}\right), F \in Y_{u l}^{\sigma}(J)$ and

$$
\left\|\nabla_{x, z} \tilde{v}\right\|_{X_{u l}^{s_{0}-1}(J)}<+\infty .
$$

Then for every $z_{0} \in(-1,0)$ one has $\nabla_{x, z} \tilde{v} \in X_{u l}^{\sigma}\left(z_{0}, 0\right)$ and one can find $\mathcal{F}: \mathbf{R}^{+} \rightarrow \mathbf{R}^{+}$non-decreasing, depending only on $\left(s_{0}, s, d\right)$ such that

$$
\begin{aligned}
& \left\|\nabla_{x, z} \tilde{v}\right\|_{X_{u l}^{\sigma}\left(z_{0}, 0\right)} \\
& \quad \leq \mathcal{F}\left(\|(\eta, \psi)\|_{H_{u l}^{s_{0}+\frac{1}{2}} \times H_{u l}^{s_{0}}}\right)\left\{\|\psi\|_{H_{u l}^{\sigma+1}}+\|F\|_{Y_{u l}^{\sigma}(J)}+\left(\|\eta\|_{H_{u l}^{s+\frac{1}{2}}}+1\right)\left\|\nabla_{x, z} \tilde{v}\right\|_{X_{u l}^{s_{0}-1}(J)}\right\} .
\end{aligned}
$$

Corollary 3.13. Let $s_{0}>1+\frac{d}{2}$. Let $\Phi$ be defined in Proposition 3.3.

Case 1. For $-\frac{1}{2} \leq \sigma \leq s_{0}-1$ assume that $\eta \in H_{u l}^{s_{0}+\frac{1}{2}}\left(\mathbf{R}^{d}\right)$ satisfying (3.2) and $\psi \in H_{u l}^{\sigma+1}\left(\mathbf{R}^{d}\right)$. Then there exists $\mathcal{F}: \mathbf{R}^{+} \rightarrow \mathbf{R}^{+}$non-decreasing depending only on $\left(s_{0}, d\right)$ such that

$$
\|\widetilde{\Phi}\|_{X_{u l}^{\sigma+1}\left(z_{0}, 0\right)}+\left\|\nabla_{x, z} \widetilde{\Phi}\right\|_{X_{u l}^{\sigma}\left(z_{0}, 0\right)} \leq \mathcal{F}\left(\|\eta\|_{H_{u l}^{s_{0}+\frac{1}{2}}}\right)\|\psi\|_{H_{u l}^{\sigma+1}} .
$$

Case 2. For $s \geq s_{0}, s_{0}-1 \leq \sigma \leq s-\frac{1}{2}$ assume that $\eta \in H_{u l}^{s+\frac{1}{2}}\left(\mathbf{R}^{d}\right)$ satisfying (3.2) and $\psi \in H_{u l}^{\sigma+1}\left(\mathbf{R}^{d}\right)$. Then there exists $\mathcal{F}: \mathbf{R}^{+} \rightarrow \mathbf{R}^{+}$non-decreasing depending only on $\left(s_{0}, s, d\right)$ such that

$$
\begin{aligned}
& \|\widetilde{\Phi}\|_{X_{u l}^{\sigma+1}\left(z_{0}, 0\right)}+\left\|\nabla_{x, z} \widetilde{\Phi}\right\|_{X_{u l}^{\sigma}\left(z_{0}, 0\right)} \\
& \quad \leq \mathcal{F}\left(\|(\eta, \psi)\|_{\left.H_{u l}^{s_{0}+\frac{1}{2}} \times H_{u l}^{s_{0}}\right)}\right)\left\{\|\eta\|_{H_{u l}^{s+\frac{1}{2}}}+\|\psi\|_{H_{u l}^{\sigma+1}}+1\right\} .
\end{aligned}
$$

Proof. Indeed $\widetilde{\Phi}$ satisfies (3.30) with $F=0$ and it is proved in Corollary 3.9 that

$$
\left\|\nabla_{x, z} \widetilde{\Phi}\right\|_{X_{u l}^{-\frac{1}{2}}\left(z_{0}, 0\right)} \leq \mathcal{F}\left(\|\eta\|_{\left.H_{u l}^{s_{0}+\frac{1}{2}}\right)\|\psi\|_{H_{u l}^{\frac{1}{2}}}<+\infty}\right.
$$

Moreover the estimate of $\widetilde{\Phi}$ in $X_{u l}^{\sigma}\left(z_{0}, 0\right)$ is obtained by the Poincare inequality from the estimate of $\partial_{z} \widetilde{\Phi}$.

Proof of Theorem 3.10 given Corollary 3.13. Let us set

$$
U=\Lambda_{1} \widetilde{\Phi}-\nabla_{x} \rho \cdot \Lambda_{2} \widetilde{\Phi}
$$

By (3.18) we have $\left.U\right|_{z=0}=G(\eta) \psi$ and by (3.21)

$$
\partial_{z} U=-\nabla_{x} \cdot\left(\left(\partial_{z} \rho\right) \Lambda_{2} \widetilde{\Phi}\right)=-\nabla_{x} \cdot\left(\left(\partial_{z} \rho\right) \nabla_{x}-\left(\nabla_{x} \rho\right) \partial_{z}\right) \widetilde{\Phi} .
$$

Using Lemma 7.12 with $f=\chi_{q} U$, we deduce that 


$$
\left\|\chi_{q} G(\eta) \psi\right\|_{H^{\sigma}} \leq C\left[\left\|\chi_{q} U\right\|_{L^{2}\left(J, H^{\sigma+\frac{1}{2}}\right)}+\left\|\chi_{q} \partial_{z} U\right\|_{L^{2}\left(J, H^{\sigma-\frac{1}{2}}\right)}\right] .
$$

Moreover by (3.34) we have

$$
\left\|\chi_{q} \partial_{z} U\right\|_{L^{2}\left(J, H^{\sigma+\frac{1}{2}}\right)} \leq C^{\prime}\left[\left\|\left(\partial_{z} \rho\right) \nabla_{x} \widetilde{\Phi}\right\|_{L^{2}\left(J, H^{\sigma+\frac{1}{2}}\right)_{u l}}+\left\|\left(\nabla_{x} \rho\right) \partial_{z} \widetilde{\Phi}\right\|_{L^{2}\left(J, H^{\sigma+\frac{1}{2}}\right)_{u l}}\right] .
$$

Case 1. If $-\frac{1}{2} \leq \sigma \leq s_{0}-1$ we use the estimate

$$
\|f g\|_{L^{2}\left(J, H^{\sigma+\frac{1}{2}}\right)_{u l}} \leq C\|f\|_{L^{\infty}\left(J, H^{s} 0^{-\frac{1}{2}}\right)_{u l}}\|g\|_{L^{2}\left(J, H^{\sigma+\frac{1}{2}}\right)_{u l}}
$$

which follows from Proposition 7.3 with $\sigma_{0}=\sigma+\frac{1}{2}, \sigma_{1}=s_{0}-\frac{1}{2}, \sigma_{2}=\sigma+\frac{1}{2}$, the estimates on $\rho$ and Corollary 3.13.

Case 2. If $s_{0}-1 \leq \sigma \leq s-\frac{1}{2}$ we use the inequality

$$
\|f g\|_{L^{2}\left(J, H^{\sigma+\frac{1}{2}}\right)_{u l}} \leq C\left(\|f\|_{L^{\infty}\left(J, H^{s_{0}-1}\right)_{u l}}\|g\|_{X_{u l}^{\sigma}(J)}+\|g\|_{L^{\infty}\left(J, H^{s_{0}-1}\right)_{u l}}\|f\|_{X_{u l}^{\sigma}(J)}\right),
$$

the estimates on $\rho$ and again Corollary 3.13 to obtain Theorem 3.10.

Theorem 3.12 will be a consequence of the following two results.

Proposition 3.14. Let $s_{0}>1+\frac{d}{2}$. There exists $\mathcal{F}: \mathbf{R}^{+} \rightarrow \mathbf{R}^{+}$non-decreasing such that for $-1<z_{0}<z_{1}<0$, $-\frac{1}{2} \leq \sigma \leq s_{0}-1$ and $k \in \mathbf{Z}^{d}$ we have

$$
\left\|\nabla_{x, z} \tilde{v}_{k}\right\|_{X^{\sigma}\left(z_{1}, 0\right)} \leq \mathcal{F}\left(\|\eta\|_{H_{u l}^{s_{0}+\frac{1}{2}}}\right)\left\{\|\psi\|_{H_{u l}^{\sigma+1}}+\|F\|_{Y_{u l}^{\sigma}(J)}+\left\|\nabla_{x, z} \tilde{v}_{k}\right\|_{X^{-\frac{1}{2}\left(z_{0}, 0\right)}}\right\},
$$

where $\tilde{v}_{k}=\chi_{k} \tilde{v}$.

Proposition 3.15. Let $s_{0}>1+\frac{d}{2}$, and $s \geq s_{0}$. Then there exists $\mathcal{F}: \mathbf{R}^{+} \rightarrow \mathbf{R}^{+}$non-decreasing such that for $-1<$ $z_{0}<z_{1}<0, s_{0}-1 \leq \sigma \leq s-\frac{1}{2}$ and $k \in \mathbf{Z}^{d}$ we have

$$
\left\|\nabla_{x, z} \tilde{v}_{k}\right\|_{X^{\sigma}\left(z_{1}, 0\right)} \leq \mathcal{F}\left(\|\eta\|_{H_{u l}^{s_{0}+\frac{1}{2}}}\right)\left\{\|\psi\|_{H_{u l}^{\sigma+1}}+\|F\|_{Y_{u l}^{\sigma}(J)}+\left(1+\|\eta\|_{H_{u l}^{s+\frac{1}{2}}}\right)\left\|\nabla_{x, z} \tilde{v}_{k}\right\|_{X_{u l}^{s_{0}-1}\left(z_{0}, 0\right)}\right\},
$$

where $\tilde{v}_{k}=\chi_{k} \tilde{v}$.

We shall prove these two results by induction on $\sigma$ and by the same method. However we have to distinguish them since we want the right hand side of these estimates to be linear with respect to the higher norms of $(\psi, \eta)$. Since $\left(\mathcal{H}_{-\frac{1}{2}}\right)$ and $\left(\mathcal{K}_{s_{0}-1}\right)$ are trivially satisfied if $\mathcal{F} \geq 1$ these propositions will be a consequence of the following one.

Proposition 3.16. Case 1. Let $s_{0}>1+\frac{d}{2}$. If $\left(\mathcal{H}_{\sigma}\right)$ is satisfied for some $-\frac{1}{2} \leq \sigma \leq s_{0}-1$ then $\left(\mathcal{H}_{\sigma+\frac{1}{2}}\right)$ is true as long as $\sigma+\frac{1}{2} \leq s_{0}-1$.

Case 2. Let $s_{0}>1+\frac{d}{2}$ and $s \geq s_{0}$. If $\left(\mathcal{K}_{\sigma}\right)$ is satisfied for some $s_{0}-1 \leq \sigma \leq s-\frac{1}{2}$ then $\left(\mathcal{K}_{\sigma+\frac{1}{2}}\right)$ is true as long as $\sigma+\frac{1}{2} \leq s-\frac{1}{2}$.

In the sequel, Case 1 will refer to Proposition 3.14 and Case 2 to Proposition 3.15.

\subsection{Nonlinear estimates}

We begin by estimating the coefficients $\alpha, \beta, \gamma$, defined in (3.23). We set $J=\left(z_{0}, 0\right)$.

Lemma 3.17. Case 1. Let $s_{0}>1+\frac{d}{2}$. Then there exists $\mathcal{F}: \mathbf{R}^{+} \rightarrow \mathbf{R}^{+}$non-decreasing such that

$$
\|\alpha\|_{X_{u l}^{s_{0}-\frac{1}{2}}(J)}+\|\beta\|_{X_{u l}^{s_{0}-\frac{1}{2}}(J)}+\|\gamma\|_{X_{u l}^{s_{0}-\frac{3}{2}}(J)} \leq \mathcal{F}\left(\|\eta\|_{H_{u l}^{s_{0}+\frac{1}{2}}}\right) .
$$


Case 2. Let $s_{0}>1+\frac{d}{2}$. Then for $s \geq s_{0}$ there exists $\mathcal{F}_{1}: \mathbf{R}^{+} \rightarrow \mathbf{R}^{+}$non-decreasing such that

$$
\|\alpha\|_{X_{u l}^{s-\frac{1}{2}}(J)}+\|\beta\|_{X_{u l}^{s-\frac{1}{2}}(J)}+\|\gamma\|_{X_{u l}^{s-\frac{3}{2}}(J)} \leq \mathcal{F}_{1}\left(\|\eta\|_{H_{u l}^{s_{0}+\frac{1}{2}}}\right)\left(\|\eta\|_{H_{u l}^{s+\frac{1}{2}}}+1\right) .
$$

Proof. Since

$$
\rho=(1+z) e^{\delta z\left\langle D_{x}\right\rangle} \eta-z\left(e^{-\delta(1+z)\left\langle D_{x}\right\rangle} \eta-h\right)
$$

Corollary 7.11 shows that for all $t \in \mathbf{R}, k \in \mathbf{N}$ and all $a \in S_{1,0}^{m}$ we have

$$
\left\|\partial_{z}^{k} a(D) \rho\right\|_{X_{u l}^{t}(J)} \leq C\left(1+\|\eta\|_{H_{u l}^{t+m+k}}\right) .
$$

Then according to (3.23) we write

$$
\alpha=\left(\partial_{z} \rho\right)^{2}-\left(\partial_{z} \rho\right)^{2} G_{1}\left(\nabla_{x} \rho\right), \quad G_{1}(\xi)=\frac{|\xi|^{2}}{1+|\xi|^{2}} .
$$

Case 1: The estimate for $\alpha$ follows from (7.3) and (7.5) with $\mu=s_{0}-\frac{1}{2}$. The estimate for $\beta$ is similar. Now we can write

$$
\gamma=\frac{\partial_{z}^{2} \rho}{\partial_{z} \rho}+\left(\partial_{z} \rho-\left(\partial_{z} \rho\right) G_{1}\left(\nabla_{x} \rho\right)\right) \Delta_{x} \rho+G_{2}\left(\nabla_{x} \rho\right) \cdot \nabla_{x} \partial_{z} \rho, \quad G_{2}(\xi)=\frac{\xi}{1+|\xi|^{2}} .
$$

To estimate $\gamma$ we first use the embedding

$$
X_{u l}^{s_{0}-\frac{1}{2}} \times X_{u l}^{s_{0}-\frac{3}{2}} \subset X_{u l}^{s_{0}-\frac{3}{2}}
$$

which is a consequence of Lemma 7.5 with $p=+\infty, \sigma_{0}=\sigma_{1}=s_{0}-\frac{3}{2}, \sigma_{2}=s_{0}-\frac{1}{2}$ and $p=2, \sigma_{0}=\sigma_{2}=s_{0}-1$, $\sigma_{1}=s_{0}-\frac{1}{2}$. Then we use (7.5) and (3.35).

Case 2: The estimates of $\alpha$ and $\beta$ follow from (7.3) and (7.5) with $\mu=s-\frac{1}{2}$ and from (3.35). The estimate of $\gamma$ follows from (7.4) with $\mu=s-\frac{1}{2}$ and (3.35) with $t=s_{0}-\frac{3}{2}, m+k=2$.

According to (3.30) we have

$$
\left\{\begin{array}{l}
\left(\partial_{z}^{2}+\alpha \Delta_{x}+\beta \cdot \nabla_{x} \partial_{z}\right)\left(\chi_{k} \tilde{v}\right)=\chi_{k} F+F_{0}+F_{1} \\
F_{0}:=\alpha \nabla_{x} \chi_{k} \cdot \nabla_{x} \tilde{v}+\alpha\left(\Delta_{x} \chi_{k}\right) \tilde{v}+\beta \cdot \nabla_{x} \chi_{k} \partial_{z} \tilde{v} \\
F_{1}:=\gamma \chi_{k} \partial_{z} \tilde{v}
\end{array}\right.
$$

Lemma 3.18. Case 1. Let $s_{0}>1+\frac{d}{2}$. There exists $\mathcal{F}: \mathbf{R}^{+} \rightarrow \mathbf{R}^{+}$non-decreasing such that for $-\frac{1}{2} \leq \sigma \leq s_{0}-1$ with $\sigma+\frac{1}{2} \leq s_{0}-1$

$$
\sum_{j=0}^{1}\left\|F_{j}\right\|_{Y^{\sigma+\frac{1}{2}(J)}} \leq \mathcal{F}\left(\|\eta\|_{H_{u l}^{s_{0}+\frac{1}{2}}}\right)\left\|\nabla_{x, z} \tilde{v}\right\|_{X_{u l}^{\sigma}(J)}
$$

Case 2. Assume $s_{0}>1+\frac{d}{2}$. Then for all $s \geq s_{0}$ there exists $\mathcal{F}: \mathbf{R}^{+} \rightarrow \mathbf{R}^{+}$non-decreasing such that for $s_{0}-1 \leq$ $\sigma \leq s-\frac{1}{2}$ with $\sigma+\frac{1}{2} \leq s-\frac{1}{2}$ we have

$$
\sum_{j=0}^{1}\left\|F_{j}\right\|_{Y^{\sigma+\frac{1}{2}(J)}} \leq \mathcal{F}\left(\|\eta\|_{H_{u l}^{s_{0}+\frac{1}{2}}}\right)\left\{\left\|\nabla_{x, z} \tilde{v}\right\|_{X_{u l}^{\sigma}(J)}+\left(\|\eta\|_{H_{u l}^{s+\frac{1}{2}}}+1\right)\left\|\nabla_{x, z} \tilde{v}\right\|_{X_{u l}^{s_{0}-1}(J)}\right\} .
$$

Proof. Case 1: The terms $F_{0}$ and $F_{1}$ have the same structure but $F_{1}$ is worse since, according to Lemma 3.17, $\gamma$ is bounded in a weaker norm.

Let us look at $F_{1}$. We can use Proposition 7.5 with $p=2, \sigma_{0}=\sigma, \sigma_{1}=\sigma, \sigma_{2}=s_{0}-1$. Indeed we have $\sigma_{1}+\sigma_{2}>0$ since $s_{0}>1+\frac{d}{2}, \sigma_{0}=\sigma_{1}, \sigma_{0} \leq \sigma_{2}$ due to the definition of $\sigma$, eventually $\sigma_{0}<\sigma_{1}+\sigma_{2}-\frac{d}{2}$ since $s_{0}>1+\frac{d}{2}$. We obtain 
$\left\|\gamma \chi_{k} \partial_{z} \tilde{v}\right\|_{L^{2}\left(J, H^{\sigma}\right)} \leq C\left\|\tilde{\chi}_{k} \gamma\right\|_{L^{2}\left(J, H^{\left.s_{0}-1\right)}\right.}\left\|\chi_{k} \partial_{z} \tilde{v}\right\|_{L^{\infty}\left(J, H^{\sigma}\right)}$

(where $\tilde{\chi} \in C_{0}^{\infty}\left(\mathbf{R}^{d}\right)$ is equal to one on the support of $\chi$ ) and we use Lemma 3.17 to conclude.

Case 2: Using (7.4) with $\mu=\sigma-\frac{1}{2}$ we obtain

$$
\left\|\gamma \chi_{k} \partial_{z} \tilde{v}\right\|_{L^{2}\left(J, H^{\sigma}\right)} \leq C\left(\|\gamma\|_{X_{u l}^{\sigma-\frac{1}{2}}}\left\|\partial_{z} \tilde{v}\right\|_{X_{u l}^{s_{0}-1}}+\|\gamma\|_{X_{u l}^{s_{0}-\frac{3}{2}}}\left\|\partial_{z} \tilde{v}\right\|_{X_{u l}^{\sigma}}\right) .
$$

Since $\sigma-\frac{1}{2} \leq s-\frac{3}{2}$ we can use Lemma 3.17 to conclude.

Our next step is to replace the multiplication by $\alpha$ (resp. $\beta$ ) by the paramultiplication by $T_{\alpha}$ (resp. $T_{\beta}$ ). Recall that we see that Eq. (3.36) can be written as

$$
\left(\partial_{z}^{2}+\alpha \Delta_{x}+\beta \cdot \nabla_{x} \partial_{z}\right) \tilde{v}_{k}=F+F_{0}+F_{1} .
$$

Then we have the following result.

Lemma 3.19. Let $J=\left(z_{0}, 0\right), s_{0}>1+\frac{d}{2}$ and $s \geq s_{0}$. There exists $\mathcal{F}: \mathbf{R}^{+} \rightarrow \mathbf{R}^{+}$non-decreasing such that, for all $I \subset J, \tilde{v}_{k}$ satisfies the paradifferential equation

$$
\left(\partial_{z}^{2}+T_{\alpha} \Delta_{x}+T_{\beta} \cdot \nabla_{x} \partial_{z}\right) \tilde{v}_{k}=F+F_{0}+F_{1}+F_{2}
$$

for some remainder $F_{2}$ satisfying

Case 1: if $0 \leq \sigma \leq s_{0}-1$ with $\sigma+\frac{1}{2} \leq s_{0}-1$

$$
\left\|F_{2}\right\|_{Y^{\sigma+\frac{1}{2}(I)}} \leq \mathcal{F}\left(\|\eta\|_{H_{u l}^{s_{0}+\frac{1}{2}}}\right)\left\|\nabla_{x, z} \tilde{v}\right\|_{X_{u l}^{\sigma}(I)} ;
$$

Case 2: if $s_{0}-1 \leq \sigma \leq s-\frac{1}{2}$ with $\sigma+\frac{1}{2} \leq s-\frac{1}{2}$

$$
\left\|F_{2}\right\|_{Y^{\sigma+\frac{1}{2}(I)}} \leq \mathcal{F}\left(\|\eta\|_{H_{u l}^{s_{0}+\frac{1}{2}}}\right)\left(\|\eta\|_{H_{u l}^{s+\frac{1}{2}}}+1\right)\left\|\nabla_{x, z} \tilde{v}\right\|_{X_{u l}^{s_{0}-1}(J)} .
$$

Proof. Case 1: Using Proposition 7.18 with $\gamma=\sigma, r=s_{0}-\frac{1}{2}, \mu=\sigma-\frac{1}{2}$ which satisfy all the conditions we obtain

$$
\begin{aligned}
& \left\|\left(\alpha-T_{\alpha}\right) \Delta_{x} \tilde{v}_{k}\right\|_{L^{2}\left(J, H^{\sigma}\right)} \leq C\|\alpha\|_{L^{\infty}\left(J, H^{s_{0}-\frac{1}{2}}\right)_{u l}}\left\|\nabla_{x, z} \tilde{v}_{k}\right\|_{L^{2}\left(J, H^{\sigma+\frac{1}{2}}\right)}, \\
& \left\|\left(\beta-T_{\beta}\right) \cdot \nabla_{x} \partial_{z} \tilde{v}_{k}\right\|_{L^{2}\left(J, H^{\sigma}\right)} \leq C\|\beta\|_{L^{\infty}\left(J, H^{s_{0}-\frac{1}{2}}\right)_{u l}}\left\|\nabla_{x, z} \tilde{v}_{k}\right\|_{L^{2}\left(J, H^{\sigma+\frac{1}{2}}\right)}
\end{aligned}
$$

The result follows then from Lemma 3.17.

Case 2: By Theorem 2.10 in [2] we have the following estimate for $\sigma>0$

$$
\left\|\left(\alpha-T_{\alpha}\right) u\right\|_{H^{\sigma}} \leq C\|u\|_{C_{*}^{-\frac{1}{2}}}\|\alpha\|_{H^{\sigma+\frac{1}{2}}}
$$

where the space $C_{*}^{-\frac{1}{2}}$ has been defined in (2.4).

Let $\tilde{\chi} \in C_{0}^{\infty}\left(\mathbf{R}^{d}\right)$ equal to one on the support of $\chi$. We write

$$
\left(\alpha-T_{\alpha}\right) \Delta_{x} \tilde{v}_{k}=\left(\tilde{\chi}_{k} \alpha-T_{\widetilde{\chi}_{k} \alpha}\right) \Delta_{x} \tilde{v}_{k}+T_{\left(\widetilde{\chi}_{k}-1\right) \alpha} \Delta_{x} \tilde{v}_{k} .
$$

It follows from Proposition 7.18 and the above inequality that

$$
\left\|\left(\alpha-T_{\alpha}\right) \Delta_{x} \tilde{v}_{k}\right\|_{H^{\sigma}} \leq C\left\|\Delta_{x} \tilde{v}_{k}\right\|_{C_{*}^{-\frac{1}{2}}}\left\|\tilde{\chi}_{k} \alpha\right\|_{H^{\sigma+\frac{1}{2}}} .
$$

Since $H^{s_{0}-\frac{1}{2}} \subset C_{*}^{\frac{1}{2}}$ and $\sigma \leq s-1$ we obtain

$$
\left\|\left(\alpha-T_{\alpha}\right) \Delta_{x} \tilde{v}_{k}\right\|_{L^{2}\left(J, H^{\sigma}\right)} \leq C\left\|\nabla_{x} \tilde{v}\right\|_{X_{u l}^{s_{0}-1}(J)}\|\alpha\|_{X_{u l}^{s-\frac{1}{2}}(J)}
$$

which in view of Lemma 3.17 Case 2, proves (3.40). 
Then as in [2] we perform a decoupling in a forward and a backward parabolic evolution equations. Recall that $\eta \in H_{u l}^{s+\frac{1}{2}}\left(\mathbf{R}^{d}\right)$, in particular $\eta \in W^{\frac{3}{2}, \infty}\left(\mathbf{R}^{d}\right)$. We can apply Lemma 3.29 in [2] to obtain the following result.

Lemma 3.20. Let $s_{0}>1+\frac{d}{2}$. There exist two symbols a, $A$ in $\Gamma_{\frac{1}{2}}^{1}\left(\mathbf{R}^{d} \times J\right)($ see Definition 7.15$), \mathcal{F}: \mathbf{R}^{+} \rightarrow \mathbf{R}^{+}$ non-decreasing and a remainder term $F_{3}$ such that

$$
\left(\partial_{z}-T_{a(z)}\right)\left(\partial_{z}-T_{A(z)}\right) \tilde{v}_{k}=F+F_{0}+F_{1}+F_{2}+F_{3}
$$

with

$$
\sup _{z \in(-1,0)}\left(M_{\frac{1}{2}}^{1}(a(z))+M_{\frac{1}{2}}^{1}(A(z))\right) \leq \mathcal{F}\left(\|\eta\|_{H_{u l}^{s_{0}+\frac{1}{2}}\left(\mathbf{R}^{d}\right)}\right)
$$

(see (7.18)) and

$$
\left\|F_{3}\right\|_{Y^{\sigma+\frac{1}{2}(J)}} \leq \mathcal{F}\left(\|\eta\|_{H_{u l}^{s_{0}+\frac{1}{2}}\left(\mathbf{R}^{d}\right)}\right)\left\|\nabla_{x, z} \tilde{v}\right\|_{X_{u l}^{\sigma}(J)}
$$

for all $\sigma \in \mathbf{R}$.

Proof. We follow closely the proof of Lemma 3.29 in [2]. We set

$$
\begin{aligned}
& a=\frac{1}{2}\left(-i \beta \cdot \xi-\sqrt{4 \alpha|\xi|^{2}-(\beta \cdot \xi)^{2}}\right) \\
& A=\frac{1}{2}\left(-i \beta \cdot \xi+\sqrt{4 \alpha|\xi|^{2}-(\beta \cdot \xi)^{2}}\right) .
\end{aligned}
$$

We claim the there exists $c>0$ depending only on $\|\eta\|_{H_{u l}^{s_{0}+\frac{1}{2}}\left(\mathbf{R}^{d}\right)}$ such that

$$
\sqrt{4 \alpha|\xi|^{2}-(\beta \cdot \xi)^{2}} \geq c|\xi| .
$$

Indeed according to (3.23) we see by an elementary computation that

$$
4 \alpha|\xi|^{2}-(\beta \cdot \xi)^{2} \geq \frac{4\left(\partial_{z} \rho\right)^{2}}{\left(1+\left|\nabla_{x} \rho\right|^{2}\right)^{2}}|\xi|^{2} .
$$

Then our claim follows from (3.16).

Since we have $s_{0}>1+\frac{d}{2}$ we deduce from the paradifferential symbolic calculus that

$$
\left(\partial_{z}-T_{a}\right)\left(\partial_{z}-T_{A}\right)=\partial_{z}^{2}+T_{\alpha} \Delta_{x}+T_{\beta} \cdot \nabla_{x} \partial_{z}+R_{0}+R_{1}
$$

where

$$
\begin{aligned}
& R_{0}(z):=T_{a(z)} T_{A(z)}-T_{\alpha} \Delta_{x} \quad \text { is of order } \frac{3}{2} \\
& R_{1}(z):=-T_{\partial_{z} A(z)} \quad \text { is of order } \frac{3}{2}
\end{aligned}
$$

together with the estimates

$$
\sup _{z \in(-1,0)}\left(\left\|R_{0}(z)\right\|_{H^{t+\frac{3}{2}} \rightarrow H^{t}}+\left\|R_{1}(z)\right\|_{H^{t+\frac{3}{2}} \rightarrow H^{t}}\right) \leq \mathcal{F}\left(\sup _{z \in(-1,0)}\left(M_{\frac{1}{2}}^{1}(A(z))+M_{\frac{1}{2}}^{1}(a(z))\right)\right) .
$$

Now the semi-norms $M_{\frac{1}{2}}^{1}(A(z))$ and $M_{\frac{1}{2}}^{1}(a(z))$ are bounded by the $W^{\frac{1}{2}, \infty}\left(\mathbf{R}^{d}\right)$ norms of $\alpha$ and $\beta$. Since for $f=\alpha, \beta$ we have

$$
\|f(z)\|_{W^{\frac{1}{2}, \infty}\left(\mathbf{R}^{d}\right)} \leq C\|f(z)\|_{H_{u l}^{s_{0}-\frac{1}{2}}\left(\mathbf{R}^{d}\right)}
$$

we deduce from Lemma 3.17 and the fact that the symbols of $R_{j}$ vanish near the origin that for $j=0,1$

$$
\left\|R_{j}(z) \tilde{v}_{k}\right\|_{H^{\sigma}} \leq \mathcal{F}\left(\|\eta\|_{H_{u l}^{s_{0}+\frac{1}{2}}}\right)\left\|\nabla_{x} \tilde{v}_{k}\right\|_{H^{\sigma+\frac{1}{2}}} .
$$

The proof is complete. 


\subsection{Proof of Proposition 3.16}

Case 1. Assume that $\left(\mathcal{H}_{\sigma}\right)$ is satisfied, which means that there exists $I_{0}=\left(z_{0}, 0\right)$ such that

$$
\left\|\nabla_{x, z} \tilde{v}_{k}\right\|_{X^{\sigma}\left(I_{0}\right)} \leq \mathcal{F}\left(\|\eta\|_{H_{u l}^{s_{0}+\frac{1}{2}}}\right)\left\{\|\psi\|_{H_{u l}^{\sigma+1}}+\|F\|_{Y_{u l}^{\sigma}(J)}+\left\|\nabla_{x, z} \tilde{v}\right\|_{X_{u l}^{-\frac{1}{2}}(J)}\right\} .
$$

From this estimate and the Poincaré inequality we deduce that

$$
\left\|\nabla_{x, z} \tilde{v}\right\|_{X^{\sigma}\left(I_{0}\right)} \leq \mathcal{F}\left(\|\eta\|_{H_{u l}^{s_{0}+\frac{1}{2}}}\right)\left\{\|\psi\|_{H_{u l}^{\sigma+1}}+\|F\|_{Y_{u l}^{\sigma}(J)}+\left\|\nabla_{x, z} \tilde{v}\right\|_{X_{u l}^{-\frac{1}{2}}(J)}\right\} .
$$

Let $z_{1}>z_{0}$ and $I_{1}=\left(z_{1}, 0\right)$. We want to prove that

$$
\left\|\nabla_{x, z} \tilde{v}_{k}\right\|_{X^{\sigma+\frac{1}{2}\left(I_{1}\right)}} \leq \mathcal{F}_{1}\left(\|\eta\|_{H_{u l}^{s_{0}+\frac{1}{2}}}\right)\left\{\|\psi\|_{H_{u l}^{\sigma+\frac{3}{2}}}+\|F\|_{Y_{u l}^{\sigma+\frac{1}{2}(J)}}+\left\|\nabla_{x, z} \tilde{v}\right\|_{X_{u l}^{-\frac{1}{2}}(J)}\right\} .
$$

Introduce a cutoff function $\theta$ such that $\theta\left(z_{0}\right)=0, \theta(z)=1$ for $z \geq z_{1}$. Set

$$
\tilde{w}_{k}(z, \cdot):=\theta(z)\left(\partial_{z}-T_{A}\right) \tilde{v}_{k}(z, \cdot) .
$$

It follows from Lemma 3.20 for $z \geq z_{0}$ that

$$
\partial_{z} \tilde{w}_{k}-T_{a} \tilde{w}_{k}=\theta(z)\left(F+\sum_{j=0}^{3} F_{j}\right)+F_{4}
$$

where

$$
F_{4}=\theta^{\prime}(z)\left(\partial_{z}-T_{A}\right) \tilde{v}_{k} .
$$

We deduce from Lemma 3.18, Lemma 3.19, Lemma 3.20 and (3.47) that

$$
\sum_{j=0}^{3}\left\|\theta F_{j}\right\|_{Y^{\sigma+\frac{1}{2}\left(I_{0}\right)}} \leq \mathcal{F}\left(\|\eta\|_{H_{u l}^{s_{0}+\frac{1}{2}}}\right)\left\{\|F\|_{Y_{u l}^{\sigma+\frac{1}{2}}(J)}+\left\|\nabla_{x, z} \tilde{v}\right\|_{X_{u l}^{-\frac{1}{2}}(J)}+\|\psi\|_{H_{u l}^{\sigma+1}}\right\}
$$

and we see easily using (3.46) that

$$
\left\|F_{4}\right\|_{Y^{\sigma+\frac{1}{2}\left(I_{0}\right)}} \leq \mathcal{F}\left(\|\eta\|_{H_{u l}^{s_{0}+\frac{1}{2}}}\right)\left\{\|\psi\|_{H_{u l}^{\sigma+1}}+\|F\|_{Y_{u l}^{\sigma+\frac{1}{2}}(J)}+\left\|\nabla_{x, z} \tilde{v}\right\|_{X_{u l}^{-\frac{1}{2}}(J)}\right\} .
$$

Now using Proposition 2.18 in [2] and (3.50), (3.51) and (3.52) we see, since $\left.\tilde{w}_{k}\right|_{z=z_{0}}=0$, that

$$
\left\|\tilde{w}_{k}\right\|_{X^{\sigma+\frac{1}{2}\left(I_{0}\right)}} \leq \mathcal{F}\left(\|\eta\|_{H_{u l}^{s_{0}+\frac{1}{2}}}\right)\left\{\|\psi\|_{H_{u l}^{\sigma+1}}+\|F\|_{Y_{u l}^{\sigma+\frac{1}{2}}(J)}+\left\|\nabla_{x, z} \tilde{v}\right\|_{X_{u l}^{-\frac{1}{2}}(J)}\right\} .
$$

Now notice that on $I_{1}$ we have $\theta(z)=1$ so that

$$
\left(\partial_{z}-T_{A}\right) \tilde{v}_{k}=\tilde{w}_{k} .
$$

We may apply again Proposition 2.18 in [2] and write

$$
\begin{aligned}
\left\|\nabla_{x} \tilde{v}_{k}\right\|_{X^{\sigma+\frac{1}{2}\left(I_{1}\right)}} & \leq\left\|\tilde{v}_{k}\right\|_{X^{\sigma+1+\frac{1}{2}}\left(I_{1}\right)} \\
& \leq \mathcal{F}\left(\|\eta\|_{H_{u l}^{s_{0}+\frac{1}{2}}}\right)\left(\left\|\tilde{w}_{k}\right\|_{Y^{\sigma+\frac{3}{2}\left(I_{1}\right)}}+\|\psi\|_{H_{u l}^{\sigma+\frac{3}{2}}}\right) \\
& \leq \mathcal{F}\left(\|\eta\|_{H_{u l}^{s_{0}+\frac{1}{2}}}\right)\left(\left\|\tilde{w}_{k}\right\|_{X^{\sigma+\frac{1}{2}\left(I_{1}\right)}}+\|\psi\|_{H_{u l}^{\sigma+\frac{3}{2}}}\right) \\
& \leq \mathcal{F}\left(\|\eta\|_{H_{u l}^{s_{0}+\frac{1}{2}}}\right)\left(\|\psi\|_{H_{u l}^{\sigma+\frac{3}{2}}}+\|F\|_{Y_{u l}^{\sigma+\frac{1}{2}}(J)}+\left\|\nabla_{x, z} \tilde{v}\right\|_{X_{u l}^{-\frac{1}{2}}(J)}\right) .
\end{aligned}
$$

The same estimate for $\partial_{z} \tilde{v}_{k}$ follows then from (3.54) and (3.53). Thus we have proved (3.48) which completes the induction.

Case 2. Assuming that $\left(\mathcal{K}_{\sigma}\right)$ is true, the exact same method shows that $\left(\mathcal{K}_{\sigma+\frac{1}{2}}\right)$ holds as long as $\sigma+\frac{1}{2} \leq s-\frac{1}{2}$. Details are left to the reader. 


\subsection{Proof of Theorem 3.11}

Let $0 \leq t \leq s-\frac{1}{2}$. Recall that

$$
G(\eta) \psi=g_{1} \partial_{z} \widetilde{\Phi}-\left.g_{2} \cdot \nabla_{x} \widetilde{\Phi}\right|_{z=0}, \quad g_{1}=\frac{1+\left|\nabla_{x} \rho\right|^{2}}{\partial_{z} \rho}, \quad g_{2}=\nabla_{x} \rho .
$$

We shall set

$$
\left.g_{j}\right|_{z=0}=g_{j}^{0}, \quad j=1,2,\left.\quad A\right|_{z=0}=A_{0},\left.\quad a\right|_{z=0}=a_{0} .
$$

We recall that we have set $\chi_{k} \widetilde{\Phi}=\widetilde{\Phi}_{k}$ where $\left.\widetilde{\Phi}\right|_{z=0}=\psi$ and $\tilde{w}_{k}=\left(\partial_{z}-T_{A}\right) \widetilde{\Phi}_{k}$ (see (3.49)) for $z \in I_{1}$. It follows that we can write

$$
\begin{aligned}
\chi_{k} G(\eta) \psi & =\left.g_{1}^{0}\left(\partial_{z} \widetilde{\Phi}_{k}\right)\right|_{z=0}-\chi_{k} g_{2}^{0} \cdot \nabla_{x} \psi \\
& =\left.g_{1}^{0} \tilde{w}_{k}\right|_{z=0}+g_{1}^{0}\left[T_{A_{0}}, \chi_{k}\right] \psi+\chi_{k}\left(g_{1}^{0} T_{A_{0}}-g_{2}^{0} \cdot \nabla_{x}\right) \psi .
\end{aligned}
$$

We shall set

$$
R_{1}=\left(\tilde{\chi}_{k} g_{1}^{0}\right)\left[T_{A_{0}}, \chi_{k}\right] \psi, \quad R_{2}=\left.\left(\tilde{\chi}_{k} g_{1}^{0}\right) \tilde{w}_{k}\right|_{z=0}
$$

where $\tilde{\chi} \in C_{0}^{\infty}\left(\mathbf{R}^{d}\right)$ is equal to one on the support of $\chi$ so

$$
\chi_{k} G(\eta) \psi=\chi_{k}\left(g_{1}^{0} T_{A_{0}}-g_{2}^{0} \cdot \nabla_{x}\right) \psi+R_{1}+R_{2} .
$$

Let us set $U=\left[T_{A_{0}}, \chi_{k}\right] \psi$. By the symbolic calculus, since $H_{u l}^{s_{0}+\frac{1}{2}} \subset W^{\frac{1}{2}, \infty}$ we have for all $\sigma \in \mathbf{R}$

$$
\|U\|_{H^{\sigma}} \leq \mathcal{F}\left(\|\eta\|_{H_{u l}^{s_{0}+\frac{1}{2}}}\right)\|\psi\|_{H_{u l}^{\sigma+\frac{1}{2}}} \text {. }
$$

If $0 \leq t \leq s_{0}-\frac{1}{2}$ the product law in Proposition 7.3 gives

$$
\left\|\tilde{\chi}_{k} g_{1}^{0} U\right\|_{H^{t}} \leq\left\|\tilde{\chi}_{k} g_{1}^{0}\right\|_{H^{s_{0}-\frac{1}{2}}}\|U\|_{H^{t}} \leq \mathcal{F}\left(\|\eta\|_{H_{u l}^{s_{0}+\frac{1}{2}}}\right)\|U\|_{H^{t}} .
$$

If $s_{0}-\frac{1}{2} \leq t \leq s-\frac{1}{2}$ we use the estimation

$$
\left\|\tilde{\chi}_{k} g_{1}^{0} U\right\|_{H^{t}} \leq C\left(\left\|\tilde{\chi}_{k} g_{1}^{0}\right\|_{H^{s_{0}-1}}\|U\|_{H^{t}}+\left\|\tilde{\chi}_{k} g_{1}^{0}\right\|_{H^{t}}\|U\|_{H^{s_{0}-1}}\right) .
$$

Therefore using (3.58) and (7.5) we obtain

$$
\left\|\tilde{\chi}_{k} g_{1}^{0} U\right\|_{H^{t}} \leq \mathcal{F}\left(\|(\eta, \psi)\|_{H_{u l}^{s_{0}+\frac{1}{2}} \times H_{u l}^{s_{0}+\frac{1}{2}}}\right)\left\{\|\eta\|_{H_{u l}^{s+\frac{1}{2}}}+\|\psi\|_{H_{u l}^{t+\frac{1}{2}}}+1\right\} .
$$

It follows that we have

$$
\left\|R_{1}\right\|_{H^{t}} \leq \mathcal{F}\left(\|(\eta, \psi)\|_{H_{u l}^{s_{0}+\frac{1}{2}} \times H_{u l}^{s_{0}+\frac{1}{2}}}\right)\left\{\|\eta\|_{H_{u l}^{s+\frac{1}{2}}}+\|\psi\|_{H_{u l}^{t+\frac{1}{2}}}+1\right\} .
$$

By the same argument as above we have

$$
\left\|R_{2}\right\|_{H^{t}} \leq \mathcal{F}\left(\|\eta\|_{H_{u l}^{s_{0}+\frac{1}{2}}}\right)\left\{\left\|\left.\tilde{w}_{k}\right|_{z=0}\right\|_{H_{u l}^{t}}+\|\eta\|_{H_{u l}^{s+\frac{1}{2}}}\left\|\left.\tilde{w}_{k}\right|_{z=0}\right\|_{H_{u l}^{s_{0}-1}}\right\} .
$$

Now using (3.53) with $\sigma=t-\frac{1}{2}$ we obtain in particular

$$
\left\|\tilde{w}_{k}\right\|_{L^{2}\left(I_{1}, H^{t+\frac{1}{2}}\right)} \leq \mathcal{F}\left(\|\eta\|_{H_{u l}^{s_{0}+\frac{1}{2}}}\right)\left(\|\psi\|_{H_{u l}^{t+\frac{1}{2}}}+1\right) .
$$

Moreover we deduce from (3.50) that

$$
\left\|\partial_{z} \tilde{w}_{k}\right\|_{L^{2}\left(I_{1}, H^{\left.t-\frac{1}{2}\right)}\right.} \leq\left\|T_{a} \tilde{w}_{k}\right\|_{L^{2}\left(I_{1}, H^{\left.t-\frac{1}{2}\right)}\right.}+\sum_{j=0}^{4}\left\|F_{j}\right\|_{L^{2}\left(I_{1}, H^{t-\frac{1}{2}}\right)} .
$$


It follows from (3.61) and the estimates already obtained on the $F_{j}$ 's that

$$
\left\|\partial_{z} \tilde{w}_{k}\right\|_{L^{2}\left(I_{1}, H^{t-\frac{1}{2}}\right)} \leq \mathcal{F}\left(\|\eta\|_{H_{u l}^{s_{0}+\frac{1}{2}}}\right)\left(\|\psi\|_{H_{u l}^{t+\frac{1}{2}}}+1\right) .
$$

Applying Lemma 7.12 to $\chi_{q} \tilde{w}_{k}$ we obtain, for $0 \leq t \leq s-\frac{1}{2}$

$$
\left\|\left.\tilde{w}_{k}\right|_{z=0}\right\|_{H_{u l}^{t}} \leq \mathcal{F}\left(\|\eta\|_{H_{u l}^{s_{0}+\frac{1}{2}}}\right)\left(\|\psi\|_{H_{u l}^{t+\frac{1}{2}}}+1\right) .
$$

Combining with (3.60) we obtain

$$
\left\|R_{2}\right\|_{H^{t}} \leq \mathcal{F}\left(\|(\eta, \psi)\|_{H_{u l}^{s_{0}+\frac{1}{2}} \times H_{u l}^{s_{0}+\frac{1}{2}}}\right)\left\{\|\eta\|_{H_{u l}^{s+\frac{1}{2}}}+\|\psi\|_{H_{u l} t+\frac{1}{2}}+1\right\} .
$$

Now we have

$$
\begin{aligned}
& \chi_{k}\left(g_{1}^{0} T_{A_{0}}-g_{2}^{0} \cdot \nabla_{x}\right) \psi=\chi_{k} T_{g_{1}^{0} A_{0}-i \xi \cdot g_{2}^{0}} \psi+R_{3}+R_{4}, \\
& R_{3}=\chi_{k}\left\{\left(g_{1}^{0}-T_{g_{1}^{0}}\right) T_{A_{0}} \psi-\left(g_{2}^{0}-T_{g_{2}^{0}}\right) \cdot \nabla_{x} \psi\right\} \\
& R_{4}=\chi_{k}\left\{T_{g_{1}^{0}} T_{A_{0}}-T_{g_{1}^{0} A_{0}}\right\} .
\end{aligned}
$$

If $0 \leq t \leq s_{0}-\frac{1}{2}$ we use Proposition 7.18 with $\gamma=t, r=s_{0}-\frac{1}{2}, \mu=t-\frac{1}{2}$ which satisfy the conditions and we obtain

$$
\left\|\chi_{k}\left(g_{1}^{0}-T_{g_{1}^{0}}\right) T_{A_{0}} \psi\right\|_{H^{t}} \leq C\left\|g_{1}^{0}\right\|_{H_{u l}^{s_{0}-\frac{1}{2}}}\left\|T_{A_{0}} \psi\right\|_{H_{u l}^{t-\frac{1}{2}}}
$$

and an analogue estimate for the term containing $g_{2}^{0}$, from which we deduce

$$
\left\|R_{3}\right\|_{H^{t}} \leq \mathcal{F}\left(\|\eta\|_{H_{u l}^{s_{0}+\frac{1}{2}}}\right)\|\psi\|_{H_{u l}^{t+\frac{1}{2}}} \text {. }
$$

From Theorem 7.16(ii) with $\rho=\frac{1}{2}$ we have

$$
\left\|R_{4}\right\|_{H^{t}} \leq \mathcal{F}\left(\|\eta\|_{H_{u l}^{s_{0}+\frac{1}{2}}}\right)\|\psi\|_{H_{u l}^{t+\frac{1}{2}}} .
$$

Summing up, using (3.57), (3.59), (3.64), (3.66), and (3.67), we obtain

$$
\chi_{k} G(\eta) \psi=\chi_{k} T_{g_{1}^{0} A_{0}-i \xi \cdot g_{2}^{0}} \psi+R_{5}
$$

with

$$
\left\|R_{5}\right\|_{H^{t}} \leq \mathcal{F}\left(\|(\eta, \psi)\|_{H_{u l}^{s_{0}+\frac{1}{2}} \times H_{u l}^{s_{0}+\frac{1}{2}}}\right)\left\{\|\eta\|_{H_{u l}^{s+\frac{1}{2}}}+\|\psi\|_{H_{u l}+\frac{1}{2}}+1\right\} .
$$

So Theorem 3.11 follows from the fact that

$$
g_{1}^{0} A_{0}-i \xi \cdot g_{2}^{0}=\sqrt{\left(1+\left|\nabla_{x} \eta\right|^{2}\right)|\xi|^{2}-\left(\nabla_{x} \eta \cdot \xi\right)^{2}} .
$$

\section{A priori estimates in the uniformly local Sobolev space}

\subsection{Reformulation of the equations}

We introduce the following unknowns

$$
\zeta=\nabla_{x} \eta, \quad B=\left.\left(\partial_{y} \Phi\right)\right|_{y=\eta}, \quad V=\left.\left(\nabla_{x} \Phi\right)\right|_{y=\eta}, \quad a=-\left.\left(\partial_{y} P\right)\right|_{y=\eta}
$$

where $\Phi$ is the velocity potential and the pressure $P$ is given by

$$
P=Q-\frac{1}{2}\left|\nabla_{x, y} \Phi\right|^{2}-g y,
$$

where $Q$ is obtained from $B, V, \eta$ by solving a variational problem (see Section 4.2 below for details).

We begin by a useful formula. 
Lemma 4.1. Let $I=[0, T]$ and $s_{0}>1+\frac{d}{2}$. For all $s \geq s_{0}$ one can find $\mathcal{F}: \mathbf{R}^{+} \rightarrow \mathbf{R}^{+}$non-decreasing such that

$$
\begin{aligned}
& G(\eta) B=-\operatorname{div} V+\gamma, \quad \text { with } \\
& \|\gamma\|_{L^{\infty}\left(I, H^{s-\frac{1}{2}}\right)_{u l}} \leq \mathcal{F}\left(\|(\eta, \psi)\|_{L^{\infty}\left(I, H^{s_{0}+\frac{1}{2}} \times H^{s_{0}+\frac{1}{2}}\right)_{u l}}\right)\left\{1+\|\eta\|_{L^{\infty}\left(I, H^{s+\frac{1}{2}}\right)_{u l}}\right\},
\end{aligned}
$$

where $H^{\sigma}=H^{\sigma}\left(\mathbf{R}^{d}\right)$.

Proof. The estimate of the lemma will be proved first with fixed $t$ which therefore will be skipped. Let $\theta$ be the variational solution of the problem

$$
\Delta_{x, y} \theta=0 \quad \text { in } \Omega,\left.\quad \theta\right|_{y=\eta(x)}=B,\left.\quad \frac{\partial \theta}{\partial v}\right|_{\Gamma}=0 .
$$

Then $G(\eta) B=\left.\left(\partial_{y} \theta-\nabla_{x} \eta \cdot \nabla_{x} \theta\right)\right|_{y=\eta(x)}$. On the other hand since $V_{i}(x)=\partial_{i} \Phi(x, \eta(x))$ we have

$$
\begin{aligned}
\operatorname{div} V & =\left(\Delta_{x} \Phi+\nabla_{x} \eta \cdot \nabla_{x} \partial_{y} \Phi\right)(x, \eta(x)) \\
& =\left(-\partial_{y}^{2} \Phi+\nabla_{x} \eta \cdot \nabla_{x} \partial_{y} \Phi\right)(x, \eta(x)) \\
& =-\left(\partial_{y}-\nabla_{x} \eta \cdot \nabla_{x}\right) \partial_{y} \Phi(x, \eta(x)) .
\end{aligned}
$$

It follows that

$$
G(\eta) B+\operatorname{div} V=\gamma, \quad \gamma=\left(\partial_{y}-\nabla_{x} \eta \cdot \nabla_{x}\right)\left(\theta-\partial_{y} \Phi\right)(x, \eta(x)) .
$$

Setting $\Theta=\theta-\partial_{y} \Phi$ we see that $\Delta_{x, y} \Theta=0$ in $\Omega$, and $\left.\Theta\right|_{y=\eta}=0$. Therefore we may apply Theorem 3.12 with $\sigma=s-\frac{1}{2}$ to deduce that

$$
\left\|\nabla_{x, z} \widetilde{\Theta}\right\|_{X_{u l}^{s-\frac{1}{2}}\left(z_{0}, 0\right)} \leq \mathcal{F}\left(\|(\eta, \psi)\|_{H_{u l}^{s_{0}+\frac{1}{2}} \times H_{u l}^{s_{0}+\frac{1}{2}}}\right)\left(1+\|\eta\|_{H_{u l}^{s+\frac{1}{2}}}\right)
$$

where $\widetilde{\Theta}(x, z)=\Theta(x, \rho(x, z))$. Using (7.3) with $\mu=s-\frac{1}{2}$ we deduce that

$$
\left\|\nabla_{x, z} \tilde{\gamma}\right\|_{X_{u l}^{s-\frac{1}{2}}\left(z_{0}, 0\right)} \leq \mathcal{F}\left(\|(\eta, \psi)\|_{H_{u l}^{s_{0}+\frac{1}{2}} \times H_{u l}^{s_{0}+\frac{1}{2}}}\right)\left(1+\|\eta\|_{H_{u l}^{s+\frac{1}{2}}}\right) .
$$

Now using the equation satisfied by $\Theta$, Lemma 3.17 and Lemma 7.12 we obtain the desired conclusion.

Proposition 4.2. Let $s_{0}>1+\frac{d}{2}$. Then for all $s \geq s_{0}$ we have

$$
\begin{aligned}
& \left(\partial_{t}+V \cdot \nabla_{x}\right) B=a-g, \\
& \left(\partial_{t}+V \cdot \nabla_{x}\right) V+a \zeta=0, \\
& \left(\partial_{t}+V \cdot \nabla_{x}\right) \zeta=G(\eta) V+\zeta G(\eta) B+R,
\end{aligned}
$$

where the remainder term $R=R(\eta, \psi, V, B)$ satisfies the estimate

$$
\|R\|_{L^{\infty}\left(I, H_{u l}^{s-\frac{1}{2}}\right)} \leq \mathcal{F}\left(\|(\eta, \psi, V)\|_{L^{\infty}\left(I, H^{s_{0}+\frac{1}{2}} \times H^{s_{0}+\frac{1}{2}} \times H^{s_{0}}\right)_{u l}}\right)\left(1+\|\eta\|_{L^{\infty}\left(I, H^{s+\frac{1}{2}}\right)_{u l}}\right)
$$

where $H^{\sigma}=H^{\sigma}\left(\mathbf{R}^{d}\right)$.

Proof. According to Proposition 4.3 in [2] the only point to be proved is the estimate (4.5). Let us recall how $R$ is defined. Let $\theta_{i}, \Phi$ be the variational solutions of the problems

$$
\begin{aligned}
& \Delta_{x, y} \theta_{i}=0 \quad \text { in } \Omega,\left.\quad \theta_{i}\right|_{y=\eta}=V_{i},\left.\quad \frac{\partial \theta_{i}}{\partial v}\right|_{\Gamma}=0, \quad i=1, \ldots, d \\
& \Delta_{x, y} \theta_{d+1}=0 \quad \text { in } \Omega,\left.\quad \theta_{d+1}\right|_{y=\eta}=B,\left.\quad \frac{\partial \theta_{d+1}}{\partial v}\right|_{\Gamma}=0, \\
& \Delta_{x, y} \Phi=0 \quad \text { in } \Omega,\left.\quad \Phi\right|_{y=\eta}=\psi,\left.\quad \frac{\partial \Phi}{\partial v}\right|_{\Gamma}=0 .
\end{aligned}
$$


Then (see [2, Proposition 4.3])

$$
\begin{aligned}
& R_{i}=\left.\left(\partial_{y}-\nabla_{x} \eta \cdot \nabla_{x}\right) U_{i}\right|_{y=\eta}, \quad U_{i}=\partial_{i} \Phi-\theta_{i}, \quad i=1, \ldots, d \\
& R_{d+1}=\left.\left(\partial_{y}-\nabla_{x} \eta \cdot \nabla_{x}\right) U_{d+1}\right|_{y=\eta}, \quad U_{d+1}=\partial_{y} \Phi-\theta_{d+1} .
\end{aligned}
$$

First of all for $i=1, \ldots, d$ we have $\Delta_{x, y} U_{i}=0$ in $\Omega$ and $\left.U_{i}\right|_{y=\eta}=0$ since $\left.\partial_{i} \Phi\right|_{y=\eta}=V_{i}$. Denoting by $\widetilde{U}_{i}$ the image of $U_{i}$ by the diffeomorphism (3.15) we see that $\widetilde{U}_{i}$ satisfies Eq. (3.30) with $F=\psi=0$. It follows from Theorem 3.12 wit $\sigma=s-\frac{1}{2}$ that

$$
\left\|\nabla_{x, z} \widetilde{U}_{i}\right\|_{X_{u l}^{s-\frac{1}{2}}(J)} \leq \mathcal{F}\left(\|(\eta, \psi)\|_{H_{u l}^{s_{0}+\frac{1}{2}} \times H_{u l}^{s_{0}+\frac{1}{2}}}\right)\left(1+\|\eta\|_{H_{u l}^{s+\frac{1}{2}}}\right)\left\|\nabla_{x, z} \widetilde{U}_{i}\right\|_{X_{u l}^{-\frac{1}{2}}(J)} .
$$

We are left with the condition (3.31), that is,

$$
\left\|\nabla_{x, z} \widetilde{U}_{i}\right\|_{X_{u l}^{-\frac{1}{2}}(J)}<+\infty .
$$

Indeed, since $\theta_{i}$ is the variational solution of (4.6) Corollary 3.13 shows that

$$
\left\|\nabla_{x, z} \tilde{\theta}_{i}\right\|_{X_{u l}^{-\frac{1}{2}}(J)} \leq \mathcal{F}\left(\|\eta\|_{H_{u l}^{s_{0}+\frac{1}{2}}}\right)\left\|V_{i}\right\|_{H_{u l}^{\frac{1}{2}}} \text {. }
$$

Now $\widetilde{\partial_{i} \Phi}=\left(\partial_{i}-\frac{\partial_{i} \rho}{\partial_{z} \rho} \partial_{z}\right) \widetilde{\Phi}$. It follows that

$$
\left\|\nabla_{x} \widetilde{\partial_{i} \Phi}\right\|_{X_{u l}^{-\frac{1}{2}(J)}} \leq\left\|\widetilde{\partial_{i} \Phi}\right\|_{X_{u l}^{\frac{1}{2}(J)}} \leq\left\|\partial_{i} \widetilde{\Phi}\right\|_{X_{u l}^{\frac{1}{2}(J)}}+\left\|\frac{\partial_{i} \rho}{\partial_{z} \rho} \partial_{z} \widetilde{\Phi}\right\|_{X_{u l}^{\frac{1}{2}(J)}} .
$$

Now we use the following facts: since $s_{0}>1+\frac{d}{2}$ one has $X_{u l}^{s_{0}-\frac{1}{2}}(J) \subset X_{u l}^{\frac{1}{2}}(J)$; moreover $X_{u l}^{s_{0}-\frac{1}{2}}(J)$ is an algebra and eventually $\left\|\frac{\partial_{i} \rho}{\partial_{z} \rho}\right\|_{X_{u l}^{s_{0}-1}(J)} \leq \mathcal{F}\left(\|\eta\|_{H_{u l}^{s_{0}+\frac{1}{2}}}\right)$. We deduce using Corollary 3.13 that

$$
\left\|\nabla_{x} \widetilde{\partial_{i} \Phi}\right\|_{X_{u l}^{-\frac{1}{2}}(J)} \leq \mathcal{F}_{1}\left(\|\eta\|_{H_{u l}^{s_{0}}+\frac{1}{2}}\right)\|\psi\|_{H_{u l}^{s_{0}+\frac{1}{2}}} \cdot
$$

To estimate the term $\left\|\partial_{z} \widetilde{\partial_{i} \Phi}\right\|_{X_{u l}^{-\frac{1}{2}}(J)}$ we follow the same path using furthermore Eq. (3.30) with $F=0$ satisfied by $\widetilde{\Phi}$. We obtain eventually

$$
\left\|\nabla_{x, z} \widetilde{U}_{i}\right\|_{X_{u l}^{-\frac{1}{2}}(J)} \leq \mathcal{F}_{2}\left(\|(\eta, \psi, V)\|_{H_{u l}^{s_{0}+\frac{1}{2}} \times H_{u l}^{s_{0}+\frac{1}{2}} \times H_{u l}^{s_{0}}}\right) .
$$

Using (4.11) we obtain

$$
\left\|\nabla_{x, z} \widetilde{U}_{i}\right\|_{X_{u l}^{s-\frac{1}{2}}(J)} \leq \mathcal{F}\left(\|(\eta, \psi, V)\|_{H_{u l}^{s_{0}+\frac{1}{2}} \times H_{u l}^{s_{0}+\frac{1}{2}} \times H_{u l}^{s_{0}}}\right)\left\{1+\|\eta\|_{H_{u l}^{s+\frac{1}{2}}}\right\} .
$$

Now from (4.9) we have

$$
R_{i}:=\left.\mathcal{R}_{i}\right|_{z=0}, \quad \mathcal{R}_{i}=\left(\frac{1+\left|\nabla_{x} \rho\right|^{2}}{\partial_{z} \rho} \partial_{z}-\nabla_{x} \rho \cdot \nabla_{x}\right) \widetilde{U}_{i}=:\left(g_{1} \partial_{z}-g_{2} \cdot \nabla_{x}\right) \widetilde{U}_{i} .
$$

Using (7.3) with $\mu=s-\frac{1}{2}$ and (4.12) with $s$ and $s_{0}$ we obtain

$$
\left\|\mathcal{R}_{i}\right\|_{X_{u l}^{s-\frac{1}{2}}(J)} \leq \mathcal{F}\left(\|(\eta, \psi, V)\|_{H_{u l}^{s_{0}+\frac{1}{2}} \times H_{u l}^{s_{0}+\frac{1}{2}} \times H_{u l}^{s_{0}}}\right)\left\{1+\|\eta\|_{H_{u l}^{s+\frac{1}{2}}}\right\} .
$$

Now we claim that

$$
\left\|\partial_{z} \mathcal{R}_{i}\right\|_{X_{u l}^{s-\frac{3}{2}}(J)} \leq \mathcal{F}\left(\|(\eta, \psi, V)\|_{H_{u l}^{s_{0}+\frac{1}{2}} \times H_{u l}^{s_{0}+\frac{1}{2}} \times H_{u l}^{s_{0}}}\right)\left\{1+\|\eta\|_{H_{u l}^{s+\frac{1}{2}}}\right\} .
$$

Indeed we can write

$$
\partial_{z} \mathcal{R}_{i}=\left(\partial_{z} A\right) \partial_{z} \widetilde{U}_{i}+\left(\partial_{z} B\right) \cdot \nabla_{x} \widetilde{U}_{i}-(\operatorname{div} B) \partial_{z} \widetilde{U}_{i}+\nabla_{x} \cdot\left(B \partial_{z} \widetilde{U}_{i}\right)+A \partial_{z}^{2} \widetilde{U}_{i} .
$$


The first three terms are bounded using (7.4) with $\mu=s-\frac{3}{2}$ and (4.12), the fourth is estimated using (7.3) with $\mu=s-\frac{1}{2}$ and (4.12), eventually for the last term we use the fact that $\partial_{z}^{2} \widetilde{U}_{i}=-\left(\alpha \Delta_{x}+\beta \cdot \nabla_{x} \partial_{z}-\gamma \partial_{z}\right) \widetilde{U}_{i}$ together with (7.3), (7.4), and (4.12). Finally from (4.14) and (4.15), using Lemma 7.12 we obtain (4.5) for $R_{i}$.

We use exactly the same argument to estimate $\left\|R_{d+1}\right\|_{H_{u l}^{s-\frac{1}{2}}\left(\mathbf{R}^{d}\right)}$. This completes the proof of Proposition 4.2.

\subsection{Estimate of the Taylor coefficient}

Proposition 4.3. Let $I=[0, T], s_{0}>1+\frac{d}{2}$. For all $s \geq s_{0}$ there exists $\mathcal{F}: \mathbf{R}^{+} \rightarrow \mathbf{R}^{+}$non-decreasing such that, with $H^{\sigma}=H^{\sigma}\left(\mathbf{R}^{d}\right)$

$$
\begin{aligned}
\|a-g\|_{L^{\infty}\left(I, H^{s-\frac{1}{2}}\right)_{u l} \leq} \leq & \mathcal{F}\left(\|(\eta, \psi, V, B)\|_{L^{\infty}\left(I, H^{s_{0}+\frac{1}{2}} \times H^{s_{0}+\frac{1}{2}} \times H^{s_{0}} \times H^{s_{0}}\right)_{u l}}\right) \\
& \times\left\{1+\|\eta\|_{L^{\infty}\left(I, H^{s+\frac{1}{2}}\right)_{u l}}+\|B\|_{L^{\infty}\left(I, H^{s}\right)_{u l}}+\|V\|_{L^{\infty}\left(I, H^{s}\right)_{u l}}\right\} .
\end{aligned}
$$

For convenience we shall set in what follows

$$
\mathcal{F}_{0}=: \mathcal{F}\left(\|(\eta, \psi, V, B)\|_{L^{\infty}\left(I, H^{s_{0}+\frac{1}{2}} \times H^{s_{0}+\frac{1}{2}} \times H^{s_{0}} \times H^{s_{0}}\right)_{u l}}\right)
$$

where $\mathcal{F}: \mathbf{R}^{+} \rightarrow \mathbf{R}^{+}$is a non-decreasing function which may change from line to line. Before giving the proof of this result let us recall how $a$ is defined. As in [2] the pressure is defined as follows. Let $Q$ be the variational solution of the problem

$$
\Delta_{x, y} Q=0 \quad \text { in } \Omega,\left.\quad Q\right|_{y=\eta}=\frac{1}{2} B^{2}+\frac{1}{2}|V|^{2}+g \eta,\left.\quad \frac{\partial Q}{\partial v}\right|_{\Gamma}=0 .
$$

Then

$$
P=Q-\frac{1}{2}\left|\nabla_{x, y} \Phi\right|^{2}-g y .
$$

It is shown in [2] that $Q=-\partial_{t} \Phi$. Then

$$
a=-\left.\partial_{y} P\right|_{y=\eta} .
$$

We deduce from (4.18), (4.19) that $P$ is solution of the problem

$$
\Delta_{x, y} P=-\left|\nabla_{x, y}^{2} \Phi\right|^{2},\left.\quad P\right|_{y=\eta}=0 .
$$

Denoting, as usual, by $\widetilde{P}, \widetilde{Q}, \widetilde{\Phi}$ the images of $P, Q, \Phi$ by the diffeomorphism (3.15) we have, using the notation (3.17),

$$
\widetilde{P}=\widetilde{Q}-\frac{1}{2}\left(\Lambda_{1} \widetilde{\Phi}\right)^{2}-\frac{1}{2}\left|\Lambda_{2} \widetilde{\Phi}\right|^{2}-g \rho
$$

and we see that $\widetilde{P}$ is a solution of the problem in $\mathbf{R}^{d} \times J$,

$$
\left(\partial_{z}^{2}+\alpha \Delta_{x}+\beta \cdot \nabla_{x} \partial_{z}-\gamma \partial_{z}\right) \widetilde{P}=-\alpha \sum_{i, j=1}^{2}\left|\Lambda_{i} \Lambda_{j} \widetilde{\Phi}\right|^{2},\left.\quad \widetilde{P}\right|_{z=0}=0
$$

Notice that we have

$$
\left.\Lambda_{1} \widetilde{\Phi}\right|_{z=0}=B,\left.\quad \Lambda_{2} \widetilde{\Phi}\right|_{z=0}=V
$$

Proof of Proposition 4.3. Below the time is fixed and we will skip it. We want to apply Theorem 3.12 with $\sigma=s-\frac{1}{2}$, so we must estimate the source term and show that the condition (3.31) is satisfied. We claim that (see (4.17))

$$
\left\|\nabla_{x, z} \widetilde{P}\right\|_{X_{u l}^{-\frac{1}{2}}(J)} \leq \mathcal{F}_{0}
$$


First of all since $Q$ is the variational solution of (4.18) we have according to Corollary 3.9

$$
\left\|\nabla_{x, z} \widetilde{Q}\right\|_{X_{u l}^{-\frac{1}{2}}} \leq \mathcal{F}\left(\|\eta\|_{H_{u l}^{s_{0}}+\frac{1}{2}}\right)\left(\left\|B^{2}\right\|_{H_{u l}^{\frac{1}{2}}}+\left\||V|^{2}\right\|_{H_{u l}^{\frac{1}{2}}}+\|\eta\|_{H_{u l}^{\frac{1}{2}}}\right) .
$$

Using the fact that $H_{u l}^{s_{0}}\left(\mathbf{R}^{d}\right)$ is an algebra contained in $H_{u l}^{\frac{1}{2}}\left(\mathbf{R}^{d}\right)$ we obtain

$$
\left\|\nabla_{x, z} \widetilde{Q}\right\|_{X_{u l}^{-\frac{1}{2}}} \leq \mathcal{F}_{0}
$$

The estimate of $\left\|\nabla_{x, z} \rho\right\|_{X_{u l}^{-\frac{1}{2}}(J)}$ by the right hand side of (4.24) is straightforward. Now, for $j=1,2$ since $X_{u l}^{s_{0}-\frac{1}{2}}(J)$ is an algebra contained in $X_{u l}^{\frac{1}{2}}(J)$ we have

$$
\left\|\nabla_{x}\left|\Lambda_{j} \widetilde{\Phi}\right|^{2}\right\|_{X_{u l}^{-\frac{1}{2}}(J)} \leq C\left\|\left|\Lambda_{j} \widetilde{\Phi}\right|^{2}\right\|_{X_{u l}^{\frac{1}{2}(J)}} \leq C^{\prime}\left\|\Lambda_{j} \widetilde{\Phi}\right\|_{X_{u l}^{s_{0 l}-\frac{1}{2}}(J)}^{2}
$$

so using Corollary 3.13 with $\sigma=s_{0}-\frac{1}{2}$ and the estimates on $\rho$ we obtain

$$
\left\|\nabla_{x}\left|\Lambda_{j} \widetilde{\Phi}\right|^{2}\right\|_{X_{u l}^{-\frac{1}{2}}(J)} \leq \mathcal{F}_{3}\left(\|(\eta, \psi)\|_{H_{u l}^{s_{0}+\frac{1}{2}} \times H_{u l}^{s_{0}+\frac{1}{2}}}\right) .
$$

The same kind of arguments shows that

$$
\left\|\partial_{z}\left|\Lambda_{j} \widetilde{\Phi}\right|^{2}\right\|_{X_{u l}^{-\frac{1}{2}}(J)} \leq \mathcal{F}_{3}\left(\|(\eta, \psi)\|_{H_{u l}^{s_{0}+\frac{1}{2}} \times H_{u l}^{s_{0}+\frac{1}{2}}}\right) .
$$

Using (4.25), (4.26), and (4.27) we obtain the claim (4.24). Now we estimate the source term $F=$ $-\alpha \sum_{i, j=1}^{2}\left|\Lambda_{i} \Lambda_{j} \widetilde{\Phi}\right|^{2}$ in Eq. (4.22). Since $s-\frac{1}{2}>\frac{d}{2}$ we can write

$$
\begin{aligned}
\|F\|_{Y_{u l}^{s-\frac{1}{2}}(J)} & \leq\|F\|_{L^{1}\left(J, H^{s-\frac{1}{2}}\right)_{u l}} \\
& \leq C\|\alpha\|_{L^{\infty}\left(J, H^{s-\frac{1}{2}}\right)_{u l}} \sum_{i, j=1}^{2}\left\|\Lambda_{i} \Lambda_{j} \widetilde{\Phi}\right\|_{L^{2}\left(J, H^{s-\frac{1}{2}}\right)_{u l}}^{2} .
\end{aligned}
$$

Since $\left(\Lambda_{1}^{2}+\Lambda_{2}^{2}\right) \widetilde{\Phi}=0$ and $\Lambda_{1}, \Lambda_{2}$ commute, we have for $j=1,2$

$$
\left(\Lambda_{1}^{2}+\Lambda_{2}^{2}\right) \Lambda_{j} \widetilde{\Phi}=0,\left.\quad\left(\Lambda_{1} \widetilde{\Phi}, \Lambda_{2} \widetilde{\Phi}\right)\right|_{z=0}=(B, V) \in H_{u l}^{s} \times H_{u l}^{s}
$$

Since we have (see (4.17))

$$
\left\|\Lambda_{j} \widetilde{\Phi}\right\|_{X_{u l}^{-\frac{1}{2}(J)}} \leq \mathcal{F}_{0}
$$

we can apply Theorem 3.12 with $\sigma=s-1$ and conclude that

$$
\left\|\nabla_{x, z} \Lambda_{j} \widetilde{\Phi}\right\|_{L^{2}\left(J, H^{s-\frac{1}{2}}\right)_{u l}} \leq \mathcal{F}_{0} \cdot\left(1+\|\eta\|_{H_{u l}^{s+\frac{1}{2}}}+\|B\|_{H_{u l}^{s}}+\|V\|_{H_{u l}^{s}}\right) .
$$

Since $\Lambda_{1}=\frac{1}{\partial_{z} \rho} \partial_{z}, \Lambda_{2}=\nabla_{x}-\frac{\nabla_{x} \rho}{\partial_{z} \rho} \partial_{z}$, using (7.3), the estimates on $\rho$, the above inequality for $s=s_{0}$ and for $s$ we obtain

$$
\left\|\Lambda_{i} \Lambda_{j} \widetilde{\Phi}\right\|_{L^{2}\left(J, H^{s-\frac{1}{2}}\left(\mathbf{R}^{d}\right)\right)_{u l}} \leq \mathcal{F}_{0} \cdot\left(1+\|\eta\|_{H_{u l}^{s+\frac{1}{2}}}+\|B\|_{H_{u l}^{s}}+\|V\|_{H_{u l}^{s}}\right) .
$$

It follows easily that

$$
\|F\|_{Y_{u l}^{s-\frac{1}{2}(J)}} \leq \mathcal{F}_{0} \cdot\left(1+\|\eta\|_{H_{u l}^{s+\frac{1}{2}}}+\|B\|_{H_{u l}^{s}}+\|V\|_{H_{u l}^{s}}\right) .
$$


Using (4.22), (4.24), (4.29), Theorem 3.12 and $X_{u l}^{s-\frac{1}{2}}(J) \subset L^{2}\left((J), H^{s}\right)_{u l}$ we obtain, using (4.17)

$$
\left\|\nabla_{x, z} \widetilde{P}\right\|_{L^{2}\left((J), H^{s}\right)_{u l}} \leq \mathcal{F}_{0} \cdot\left(1+\|\eta\|_{H_{u l}^{s+\frac{1}{2}}}+\|B\|_{H_{u l}^{s}}+\|V\|_{H_{u l}^{s}}\right) .
$$

We claim that

$$
\left\|\partial_{z}^{2} \widetilde{P}\right\|_{L^{2}\left((J), H^{s-1}\right)_{u l}} \leq \mathcal{F}_{0} \cdot\left(1+\|\eta\|_{H_{u l}^{s+\frac{1}{2}}}+\|B\|_{H_{u l}^{s}}+\|V\|_{H_{u l}^{s}}\right) .
$$

Indeed this follows from (4.22), (4.28), (7.3), (7.4), (4.30).

Noticing that $a=-\left.\frac{1}{\partial_{z} \rho} \partial_{z} \widetilde{P}\right|_{z=0}$ and applying Lemma 7.12 we obtain the conclusion of Proposition 4.3.

\subsection{Paralinearization of the system}

As in [2] for $s>1+\frac{d}{2}$ we set

$$
\left\{\begin{array}{l}
U_{s}=\left\langle D_{x}\right\rangle^{s} V+T_{\zeta}\left\langle D_{x}\right\rangle^{s} B, \\
\zeta_{s}=\left\langle D_{x}\right\rangle^{s} \zeta,
\end{array}\right.
$$

and we recall that we have set (see the statement of Theorem 3.11)

$$
\lambda(t, x, \xi):=\sqrt{\left(1+\left|\nabla_{x} \eta(t, x)\right|^{2}\right)|\xi|^{2}-\left(\nabla_{x} \eta(t, x) \cdot \xi\right)^{2}} .
$$

Proposition 4.4. Let $s_{0}>1+\frac{d}{2}$. For all $s \geq s_{0}$ there exists $\mathcal{F}: \mathbf{R}^{+} \rightarrow \mathbf{R}^{+}$non-decreasing such that

$$
\begin{aligned}
& \left(\partial_{t}+T_{V} \cdot \nabla_{x}\right) U_{s}+T_{a} \zeta_{s}=f_{1}, \\
& \left(\partial_{t}+T_{V} \cdot \nabla_{x}\right) \zeta_{s}-T_{\lambda} U_{s}=f_{2},
\end{aligned}
$$

where for each time $t \in[0, T]$

$$
\begin{aligned}
\left\|\left(f_{1}(t), f_{2}(t)\right)\right\|_{L_{u l}^{2} \times H_{u l}^{-\frac{1}{2}} \leq} & \mathcal{F}\left(\|(\eta(t), \psi(t), V(t), B(t))\|_{H_{u l}^{s_{0}+\frac{1}{2}} \times H_{u l}^{s_{0}+\frac{1}{2}} \times H_{u l}^{s_{0}} \times H_{u l}^{s_{0}}}\right) \\
& \times\left\{1+\|\eta(t)\|_{H_{u l}^{s+\frac{1}{2}}}+\|B(t)\|_{H_{u l}^{s}}+\|V(t)\|_{H_{u l}^{s}}\right\} .
\end{aligned}
$$

Proof. We follow the proof of Proposition 4.8 in [2]. First of all we shall say that a positive quantity $A(t)$ is controlled if it is bounded by the right hand side of (4.35). Here $t$ will be fixed so we will skip it, taking care that the estimates are uniform with respect to $t \in[0, T]$. We also set

$$
\mathcal{L}_{0}=\partial_{t}+T_{V} \cdot \nabla_{x}
$$

\subsubsection{Paralinearization of the first equation}

We begin by proving that

$$
\mathcal{L}_{0} V+T_{a} \zeta+T_{\zeta} \mathcal{L}_{0} B=h_{1}
$$

where $\left\|h_{1}\right\|_{H_{u l}^{s}}$ is controlled. Indeed using (4.2), (4.3) and the fact that $T_{\zeta} g=0$ we see that $h_{1}=\left(T_{V}-V\right) \cdot \nabla_{x} V+$ $T_{\zeta}\left(T_{V}-V\right) \cdot \nabla_{x} B+R(a, \zeta)$. By Proposition 7.18 with $\gamma=s, r=s, \mu=s_{0}-1$ we see that $\left\|\left(T_{V}-V\right) \cdot \nabla_{x} V\right\|_{H_{u l}^{s}} \leq$ $C\|V\|_{H_{u l}^{s}}\|V\|_{H_{u l}^{s_{0}}}$. On the other hand since $s_{0}>1+\frac{d}{2}$, Proposition 7.17 with $\alpha=s-\frac{1}{2}, \beta=s_{0}-\frac{1}{2}$ shows that

$$
\|R(a, \zeta)\|_{H_{u l}^{s}\left(\mathbf{R}^{d}\right)} \leq C\|a\|_{H_{u l}^{s-\frac{1}{2}}\left(\mathbf{R}^{d}\right)}\left\|\nabla_{x} \eta\right\|_{H_{u l}^{s_{0}-\frac{1}{2}}\left(\mathbf{R}^{d}\right)} .
$$

These estimates together with Proposition 4.3 prove that $h_{1}$ is controlled. 


\subsubsection{Higher order energy estimates}

Now we apply the operator $\left\langle D_{x}\right\rangle^{s}=\left(I-\Delta_{x}\right)^{\frac{s}{2}}$ to Eq. (4.36) and we commute. We claim that we obtain

$$
\mathcal{L}_{0}\left\langle D_{x}\right\rangle^{s} V+T_{a}\left\langle D_{x}\right\rangle^{s} \zeta+T_{\zeta} \mathcal{L}_{0}\left\langle D_{x}\right\rangle^{s} B=h_{2}
$$

where $\left\|h_{2}(t)\right\|_{L_{u l}^{2}\left(\mathbf{R}^{d}\right)}$ is controlled. Indeed this is a consequence of the following estimates

$$
\begin{gathered}
\left\|\left[T_{V} \cdot \nabla_{x},\left\langle D_{x}\right\rangle^{s}\right]\right\|_{H_{u l}^{s} \rightarrow L_{u l}^{2}} \leq C\|V\|_{W^{1, \infty}} \leq C^{\prime}\|V\|_{H_{u l}^{s_{0}}}, \\
\left\|\left[T_{a},\left\langle D_{x}\right\rangle^{s}\right]\right\|_{H_{u l}^{s-\frac{1}{2}} \rightarrow L_{u l}^{2}} \leq C\|a\|_{W^{\frac{1}{2}, \infty}} \leq C^{\prime}\|a\|_{H_{u l}^{s_{0}-\frac{1}{2}}}, \\
\left\|\left[T_{\zeta},\left\langle D_{x}\right\rangle^{s}\right]\right\|_{H_{u l}^{s-\frac{1}{2}} \rightarrow L_{u l}^{2}} \leq C\|\zeta\|_{W^{\frac{1}{2}}, \infty} \leq C^{\prime}\|\zeta\|_{H_{u l}^{s_{0}-\frac{1}{2}}},
\end{gathered}
$$

which follow from Theorem 7.16. Now Lemma 7.20 shows that

$$
\left\|\left[T_{\zeta}, \mathcal{L}_{0}\right]\left\langle D_{x}\right\rangle^{s} B\right\|_{L_{u l}^{2}} \leq C\left(\|\zeta\|_{L^{\infty}}\|V\|_{W^{1+\varepsilon, \infty}}+\left\|\mathcal{L}_{0} \zeta\right\|_{L^{\infty}}\right)\|B\|_{H_{u l}^{s}} .
$$

Since $s_{0}>1+\frac{d}{2}$ one can find $\varepsilon>0$ such that $H_{u l}^{s_{0}}\left(\mathbf{R}^{d}\right)$ is continuously embedded in $W^{1+\varepsilon, \infty}\left(\mathbf{R}^{d}\right)$. Therefore we obtain

$$
\left\|\left[T_{\zeta}, \mathcal{L}_{0}\right]\left\langle D_{x}\right\rangle^{s} B\right\|_{L_{u l}^{2}} \leq \mathcal{F}\left(\|(\eta, B, V)\|_{H_{u l}^{s_{0}+\frac{1}{2}} \times H_{u l}^{s_{0}} \times H_{u l}^{s_{0}}}\right)\|B\|_{H_{u l}^{s}}
$$

which shows that $\left\|\left[T_{\zeta}, \mathcal{L}_{0}\right]\left\langle D_{x}\right\rangle^{s} B\right\|_{L_{u l}^{2}}$ is controlled. Using (4.37) and (4.31) we obtain (4.33).

\subsubsection{Paralinearization of the second equation}

$$
\left(\partial_{t}+V \cdot \nabla_{x}\right) \zeta=G(\eta) V+\zeta G(\eta) B+R .
$$

We first replace $V$ by $T_{V}$ modulo a controlled term. To do this we use Proposition 7.18 with $\gamma=s-\frac{1}{2}, r=s$, $\mu=s_{0}-\frac{3}{2}$ and we obtain

$$
\left\|\left(V-T_{V}\right) \cdot \nabla_{x} \zeta\right\|_{H_{u l}^{s-\frac{1}{2}}} \leq C\|V\|_{H_{u l}^{s}}\|\eta\|_{H_{u l}^{s_{0}+\frac{1}{2}}} \cdot
$$

Next we paralinearize the Dirichlet-Neumann part. To achieve this paralinearization we use the analysis performed in Section 7. Using Theorem 3.11 with $t=s-\frac{1}{2}$ we can write

$$
G(\eta) V+\zeta G(\eta) B=T_{\lambda} U+\mathcal{R}
$$

where

$$
\begin{aligned}
& U=V+T_{\zeta} B \\
& \mathcal{R}=\left[T_{\zeta}, T_{\lambda}\right] B+R(\eta) V+\zeta R(\eta) B+\left(\zeta-T_{\zeta}\right) T_{\lambda} B,
\end{aligned}
$$

and

$$
\begin{aligned}
& \|R(\eta) V\|_{H_{u l}^{s-\frac{1}{2}}}+\|\zeta R(\eta) B\|_{H_{u l}^{s-\frac{1}{2}}} \\
& \quad \leq \mathcal{F}\left(\|(\eta, B, V)\|_{H_{u l}^{s_{0}+\frac{1}{2}} \times H_{u l}^{s 0} \times H_{u l}^{s_{0}}}\right)\left(1+\|\eta\|_{H_{u l}^{s+\frac{1}{2}}}+\|B\|_{H_{u l}^{s}}+\|V\|_{H_{u l}^{s}}\right) .
\end{aligned}
$$

Using again Proposition 7.18 with $\gamma=s-\frac{1}{2}, r=s-\frac{1}{2}, \mu=s_{0}-1$ and Theorem 7.16(i) we can write

$$
\left\|\left(\zeta-T_{\zeta}\right) T_{\lambda} B\right\|_{H_{u l}^{s-\frac{1}{2}}} \leq C\|\eta\|_{H_{u l}^{s+\frac{1}{2}}} M_{0}^{1}(\lambda)\|B\|_{H_{u l}^{s_{0}}} \leq \mathcal{F}\left(\|\eta\|_{H_{u l}^{s+\frac{1}{2}}}\right)\|B\|_{H_{u l}^{s_{0}}},
$$

which shows that this term is controlled. Eventually, by Theorem 7.16(ii), the term $\left\|\left[T_{\zeta}, T_{\lambda}\right] B\right\|_{H_{u l}^{s-\frac{1}{2}}}$ is also controlled. Therefore we have the equality (4.40) with $\|\mathcal{R}\|_{H_{u l}^{s-\frac{1}{2}}}$ controlled. It follows from (4.38), (4.39) and (4.40) that 


$$
\mathcal{L}_{0} \zeta=T_{\lambda} U+\mathcal{R}
$$

where $\|\mathcal{R}\|_{H_{u l}^{s-\frac{1}{2}}}$ is controlled. By commuting Eq. (4.39) with $\left\langle D_{x}\right\rangle^{s}$ we obtain Eq. (4.34). This completes the proof of Proposition 4.4.

\subsection{Symmetrization of the equations}

As in [2] before proving an $L^{2}$ estimate for our system we begin by performing a symmetrization of the nondiagonal part. Recall that $\mathcal{L}_{0}=\partial_{t}+T_{V} \cdot \nabla_{x}$.

Proposition 4.5. Introduce the symbols

$$
\gamma=\sqrt{a \lambda}, \quad q=\sqrt{\frac{a}{\lambda}}
$$

where $a$ is the Taylor coefficient and $\lambda$ is recalled in (4.32). Set $\theta_{s}=T_{q} \zeta_{s}$ and $\mathcal{L}_{0}=\partial_{t}+T_{V} \cdot \nabla_{x}$. Then

$$
\begin{aligned}
& \mathcal{L}_{0} U_{s}+T_{\gamma} \theta_{s}=F_{1} \\
& \mathcal{L}_{0} \theta_{s}-T_{\gamma} U_{s}=F_{2}
\end{aligned}
$$

where $F_{1}, F_{2}$ satisfy, with $L_{u l}^{2}=L_{u l}^{2}\left(\mathbf{R}^{d}\right), H_{u l}^{\sigma}=H_{u l}^{\sigma}\left(\mathbf{R}^{d}\right)$,

$$
\begin{aligned}
\left\|\left(F_{1}(t), F_{2}(t)\right)\right\|_{L_{u l}^{2} \times L_{u l}^{2} \leq} & \mathcal{F}\left(\|(\eta(t), B(t), V(t))\|_{H_{u l}^{s_{0}+\frac{1}{2}} \times H_{u l}^{s_{0}} \times H_{u l}^{s_{0}}}\right) \\
& \times\left(1+\|\eta(t)\|_{H_{u l}^{s+\frac{1}{2}}}+\|B(t)\|_{H_{u l}^{s}}+\|V(t)\|_{H_{u l}^{s}}\right),
\end{aligned}
$$

for some non-decreasing function $\mathcal{F}: \mathbf{R}^{+} \rightarrow \mathbf{R}^{+}$and all $t \in[0, T]$.

Proof. We follow [2]. From (4.33) and (4.34) we have

$$
\begin{aligned}
& F_{1}=: f_{1}+\left(T_{\gamma} T_{q}-T_{a}\right) \zeta_{s} \\
& F_{2}=: T_{q} f_{2}+\left(T_{q} T_{\lambda}-T_{\gamma}\right) U_{s}-\left[T_{q}, \mathcal{L}_{0}\right] \zeta_{s} .
\end{aligned}
$$

Then the proposition follows from Lemma 7.20 and from the symbolic calculus.

We can now state our $L^{2}$ estimate. Let us set with $H_{u l}^{\sigma}=H_{u l}^{\sigma}\left(\mathbf{R}^{d}\right)$

$$
\left\{\begin{array}{l}
M_{S}(0)=\|(\eta(0), \psi(0), B(0), V(0))\|_{H_{u l}^{s+\frac{1}{2}} \times H_{u l}^{s+\frac{1}{2}} \times H_{u l}^{s} \times H_{u l}^{s},} \\
M_{S}(T)=\sup _{t \in[0, T]}\|(\eta(t), \psi(t), B(t), V(t))\|_{H_{u l}^{s+\frac{1}{2}} \times H_{u l}^{s+\frac{1}{2}} \times H_{u l}^{s} \times H_{u l}^{s}} .
\end{array}\right.
$$

Proposition 4.6. There exists $\mathcal{F}: \mathbf{R}^{+} \rightarrow \mathbf{R}^{+}$non-decreasing such that
(i) $\left\|U_{S}(t)\right\|_{L_{u l}^{2}}+\left\|\theta_{S}(t)\right\|_{L_{u l}^{2}} \leq \mathcal{F}\left(M_{s_{0}}(t)\right) M_{S}(t), \quad t \in I=:[0, T]$,
(ii) $\left\|U_{s}\right\|_{L^{\infty}\left(I, L^{2}\right)_{u l}}+\left\|\theta_{s}\right\|_{L^{\infty}\left(I, L^{2}\right)_{u l}} \leq \mathcal{F}\left(T M_{s_{0}}(T)\right)\left\{M_{S}(0)+\sqrt{T} M_{S}(T)\right\}$.

Proof. (i) This follows easily from the definition of $U_{s}$ and $\theta_{s}$ given in (4.31) and in Proposition 4.5.

(ii) Let $\chi_{k}$ be as in (2.3). Then we have

$$
\left\{\begin{array}{l}
\mathcal{L}_{0}\left(\chi_{k} U_{s}\right)+T_{\gamma}\left(\chi_{k} \theta_{s}\right)=G_{1} \\
\mathcal{L}_{0}\left(\chi_{k} \theta_{s}\right)-T_{\gamma}\left(\chi_{k} U_{s}\right)=G_{2}
\end{array}\right.
$$

where $G_{1}, G_{2}$ are given by 


$$
\begin{aligned}
& G_{1}=\chi_{k} F_{1}+\left[T_{V} \cdot \nabla_{x}, \chi_{k}\right] U_{s}+\left[T_{\gamma}, \chi_{k}\right] \theta_{s} \\
& G_{2}=\chi_{k} F_{2}+\left[T_{V} \cdot \nabla_{x}, \chi_{k}\right] \theta_{s}-\left[T_{\gamma}, \chi_{k}\right] U_{s} .
\end{aligned}
$$

We claim that for all $t \in[0, T]$ we have

$$
\begin{aligned}
\left\|\left(G_{1}(t), G_{2}(t)\right)\right\|_{L^{2} \times L^{2}} \leq & \mathcal{F}\left(\|(\eta(t), B(t), V(t))\|_{H_{u l}^{s_{0}+\frac{1}{2}} \times H_{u l}^{s_{0}} \times H_{u l}^{s_{0}}}\right) \\
& \times\left(1+\|\eta(t)\|_{H_{u l}^{s+\frac{1}{2}}}+\|B(t)\|_{H_{u l}^{s}}+\|V(t)\|_{H_{u l}^{s}}\right) .
\end{aligned}
$$

According to Proposition 4.5 this is true for the terms coming from $\chi_{k} F_{j}, j=1,2$. Now according to Theorem 7.16 we have (for fixed $t$ which is skipped)

$$
\begin{aligned}
\left\|\left[T_{V} \cdot \nabla_{x}, \chi_{k}\right] U_{s}\right\|_{L^{2}} & \leq C\|V\|_{W^{1, \infty}}\left\|U_{s}\right\|_{L_{u l}^{2}} \\
& \leq C^{\prime}\|V\|_{H_{u l}^{s_{0}}}\left(\|V\|_{H_{u l}^{s}\left(\mathbf{R}^{d}\right)}+\left\|\nabla_{x} \eta\right\|_{H_{u l}^{s_{0}+\frac{1}{2}}}\|B\|_{H_{u l}^{s}}\right) \\
& \leq \mathcal{F}\left(\|(\eta, V)\|_{H_{u l}^{s_{0}+\frac{1}{2}} \times H_{u l}^{s_{0}}}\right)\left(1+\|B\|_{H_{u l}^{s}}+\|V\|_{H_{u l}^{s}}\right) .
\end{aligned}
$$

The same estimate holds for $\left\|\left[T_{V} \cdot \nabla_{x}, \chi_{k}\right] \theta_{S}\right\|_{L^{2}}$. Eventually we have

$$
\left\|\left[T_{\gamma}, \chi_{k}\right] \theta_{s}\right\|_{L^{2}} \leq \mathcal{F}\left(\|(\eta, B, V)\|_{H_{u l}^{s_{0}+\frac{1}{2}} \times H_{u l}^{s_{0}} \times H_{u l}^{s_{0}}}\right)\left(1+\|\eta\|_{H_{u l}^{s+\frac{1}{2}}}+\|B\|_{H_{u l}^{s}}+\|V\|_{H_{u l}^{s}}\right) .
$$

This proves our claim.

Now we compute the quantity $\frac{d}{d t}\left\{\left\|\chi_{k} U_{s}(t)\right\|_{L^{2}}^{2}+\left\|\chi_{k} \theta_{s}(t)\right\|_{L^{2}}^{2}\right\}$. Using Eqs. (4.42), (4.43), the point (i), the fact that

$$
\begin{aligned}
& \left\|\left(T_{V(t)} \cdot \nabla_{x}\right)^{*}+T_{V(t)} \cdot \nabla_{x}\right\|_{L^{2} \rightarrow L^{2}} \leq C M_{s_{0}}(t) \\
& \left\|T_{\gamma(t)}-\left(T_{\gamma(t)}\right)^{*}\right\|_{L^{2} \rightarrow L^{2}} \leq C M_{s_{0}}(t)
\end{aligned}
$$

and (4.45) we obtain easily (ii).

\subsection{Back to the original unknowns}

Recall that

$$
\begin{aligned}
& U_{s}=\left\langle D_{x}\right\rangle^{s} V+T_{\nabla_{x} \eta}\left\langle D_{x}\right\rangle^{s} B, \\
& \theta_{s}=T_{\sqrt{\frac{a}{\lambda}}}\left\langle D_{x}\right\rangle^{s} \nabla_{x} \eta .
\end{aligned}
$$

From the estimate in Proposition 4.6 we would like to recover estimates of the original unknowns $\psi, \eta, V, B$. We follow closely [2]. The result is as follows.

Proposition 4.7. Let $s_{0}>1+\frac{d}{2}$. For all $s \geq s_{0}$ one can find $\mathcal{F}: \mathbf{R}^{+} \rightarrow \mathbf{R}^{+}$non-decreasing such that

$$
M_{S}(T) \leq \mathcal{F}\left(M_{s_{0}}(0)+T M_{s_{0}}(T)\right)\left\{M_{S}(0)+T M_{S}(T)\right\} .
$$

The proposition will be implied by the following lemmas.

Lemma 4.8. There exists $\mathcal{F}: \mathbf{R}^{+} \rightarrow \mathbf{R}^{+}$non-decreasing such that with $I=[0, T]$ and $H_{u l}^{\sigma}=H_{u l}^{\sigma}\left(\mathbf{R}^{d}\right)$ we have

$$
\begin{aligned}
& \|\eta\|_{L^{\infty}\left(I, H^{s}\right)_{u l}}+\|(B, V)\|_{L^{\infty}\left(I, H^{s-\frac{1}{2}} \times H^{s-\frac{1}{2}}\right)_{u l}} \\
& \quad \leq \mathcal{F}\left(M_{s_{0}}(0)+\sqrt{T} M_{s_{0}}(T)\right)\left\{M_{S}(0)+\sqrt{T} M_{S}(T)\right\} .
\end{aligned}
$$


Proof. Set $\mathcal{L}_{1}=\partial_{t}+V \cdot \nabla_{x}$. According to Proposition 4.2 we have $\mathcal{L}_{1} B=a-g, \mathcal{L}_{1} V=-a \nabla_{x} \eta$ and from the definition of $V, B$ and the equations we have $\mathcal{L}_{1} \eta=B$. Then the lemma follows from Lemma 7.19 with $\mu=s$, $\mu=s-\frac{1}{2}$ and Proposition 4.3.

Lemma 4.9. Let $s_{0}>1+\frac{d}{2}$. For $s \geq s_{0}$ one can find $\mathcal{F}: \mathbf{R}^{+} \rightarrow \mathbf{R}^{+}$non-decreasing such that, with $H_{u l}^{\sigma}=H_{u l}^{\sigma}\left(\mathbf{R}^{d}\right)$, we have

$$
\begin{aligned}
\text { (i) } \quad\|\eta\|_{L^{\infty}\left(I, H^{s+\frac{1}{2}}\right)_{u l}} \leq \mathcal{F}\left(M_{s_{0}}(0)+\sqrt{T} M_{s_{0}}(T)\right)\left\{M_{S}(0)+\sqrt{T} M_{S}(T)\right\}, \\
\text { (ii) }\|(V, B)\|_{L^{\infty}\left(I, H^{s}\right)_{u l}} \leq \mathcal{F}\left(M_{s_{0}}(0)+\sqrt{T} M_{s_{0}}(T)\right)\left\{M_{S}(0)+\sqrt{T} M_{s}(T)\right\}, \\
\text { (iii) }\|\psi\|_{L^{\infty}\left(I, H^{s+\frac{1}{2}}\right)_{u l}} \leq \mathcal{F}\left(M_{s_{0}}(0)+\sqrt{T} M_{s_{0}}(T)\right)\left\{M_{S}(0)+\sqrt{T} M_{s}(T)\right\} .
\end{aligned}
$$

Proof. (i) By Lemma 4.8 it is sufficient to bound $A=\left\|\nabla_{x} \eta\right\|_{L^{\infty}\left(I, H_{u l}^{s-\frac{1}{2}}\right)}$. Recall that $q=\sqrt{\frac{a}{\lambda}}, \theta_{s}=T_{q} \zeta_{s}$, and $\zeta_{s}=\left\langle D_{x}\right\rangle^{s} \nabla_{x} \eta$. By Theorem 7.16(ii) we can write $\zeta_{s}=T_{\frac{1}{q}} T_{q} \zeta_{s}+R \zeta_{s}$ where $\|R\|_{L^{\infty}\left(I, H^{\mu}\right)_{u l} \rightarrow L^{\infty}\left(I, H^{\mu+\frac{1}{2}}\right)_{u l}} \leq$ $C\left(\|a\|_{L^{\infty}\left(I, H^{s_{0}-\frac{1}{2}}\right)_{u l}}+\|\eta\|_{L^{\infty}\left(I, H^{s_{0}}+\frac{1}{2}\right)_{u l}}\right)$. Then we have

$$
A=\left\|\left\langle D_{x}\right\rangle^{-\frac{1}{2}} \zeta_{s}\right\|_{L^{\infty}\left(I, L^{2}\right)_{u l}} \leq\left\|\left\langle D_{x}\right\rangle^{-\frac{1}{2}} T_{\frac{1}{q}} \theta_{s}\right\|_{L^{\infty}\left(I, L^{2}\right)_{u l}}+\left\|\left\langle D_{x}\right\rangle^{-\frac{1}{2}} R \zeta_{s}\right\|_{L^{\infty}\left(I, L^{2}\right)_{u l}} .
$$

Using Theorem 7.16(i), the above estimate on the norm of $R$ with $\mu=-1$, Lemma 4.8 and Proposition 4.3 we deduce that

$$
A \leq \mathcal{F}\left(M_{s_{0}}(0)+\sqrt{T} M_{s_{0}}(T)\right)\left\{\left\|\theta_{s}\right\|_{L^{\infty}\left(I, L^{2}\right)_{u l}}+\|\eta\|_{L^{\infty}\left(I, H^{s}\right)_{u l}}\right\} .
$$

Then the conclusion follows from Proposition 4.6 and Lemma 4.8.

(ii) Recall that $U=V+T_{\zeta} B$. The commutator $\left[\langle D\rangle^{s}, T_{\zeta}\right]$ is of order $s-\frac{1}{2}$ which norm from $L^{\infty}\left(I, H^{s-\frac{1}{2}}\right)_{u l}$ to $L^{\infty}\left(I, L^{2}\right)_{u l}$ is bounded by $C\|\eta\|_{L^{\infty}\left(I, C_{*}^{\frac{3}{2}}\right)}$ thus by $C^{\prime}\|\eta\|_{L^{\infty}\left(I, H^{s_{0}+\frac{1}{2}}\right)_{u l}}$. Therefore we deduce from Proposition 4.6 and Lemma 4.8 that

$$
\|U\|_{L^{\infty}\left(I, H^{s}\right)_{u l}} \leq \mathcal{F}\left(M_{s_{0}}(0)+\sqrt{T} M_{s_{0}}(T)\right)\left(M_{S}(0)+\sqrt{T} M_{S}(T)\right) .
$$

Now by Lemma 4.1 we have

$$
\begin{aligned}
\operatorname{div} U & =\operatorname{div} V+T_{\operatorname{div} \zeta} B+T_{\zeta} \cdot \nabla_{x} B=-G(\eta) B+T_{i \zeta \cdot \xi+\operatorname{div} \zeta} B+\gamma \\
& =-T_{\lambda} B+R(\eta) B+T_{i \zeta \cdot \xi+\operatorname{div} \zeta} B+\gamma=T_{e} B+T_{\operatorname{div} \zeta} B+\gamma+R(\eta) B
\end{aligned}
$$

where $e=-\lambda+i \zeta \cdot \xi$. Writing $B=T_{\frac{1}{e}} T_{e} B+\left(I-T_{\frac{1}{e}} T_{e}\right) B$ we obtain

$$
B=T_{\frac{1}{e}} \operatorname{div} U-T_{\frac{1}{e}} \gamma+S B
$$

Then using (4.46), Lemma 4.1 we obtain the desired estimate on $B$ and since $V=U-T_{\zeta} B$ the estimate on $V$ follows as well.

(iii) We have $\nabla_{x} \psi=V+B \nabla_{x} \eta$. Since the $L^{\infty}\left(I, H^{s-\frac{1}{2}}\right)_{u l}$ norm of $\left(\nabla_{x} \eta, V, B\right)$ has been already estimated, it remains to bound $\|\psi\|_{L^{\infty}\left(I, L^{2}\right)_{u l}}$. Now from (2.1) and (2.2) by a simple computation we see that

$$
\left(\partial_{t}+V \cdot \nabla_{x}\right) \psi=-g \eta+\frac{1}{2}|V|^{2}+\frac{1}{2} B^{2} .
$$

Then the conclusion follows from Lemma 7.19 with $\mu=s$ and (ii). 


\section{Contraction and well posedness}

\subsection{Contraction}

In this section we shall prove estimates on the difference of two solutions of the system described in (4.2), (4.3), (4.4) which will prove the uniqueness and also enter in the proof by contraction of the existence. Let $\left(\eta_{j}, \psi_{j}, V_{j}, B_{j}\right)$, $j=1,2$ be two solutions on $\left[0, T_{0}\right]$ of the system

$$
\left\{\begin{array}{l}
\left(\partial_{t}+V_{j} \cdot \nabla_{x}\right) B_{j}=a_{j}-g, \\
\left(\partial_{t}+V_{j} \cdot \nabla_{x}\right) V_{j}+a_{j} \zeta_{j}=0, \\
\left(\partial_{t}+V_{j} \cdot \nabla_{x}\right) \zeta_{j}=G\left(\eta_{j}\right) V_{j}+\zeta_{j} G\left(\eta_{j}\right) B_{j}+\gamma_{j}, \quad \zeta_{j}=\nabla_{x} \eta_{j},
\end{array}\right.
$$

where $\gamma_{j}$ is the remainder term given by (4.4).

Theorem 5.1. Let $s>1+\frac{d}{2}$. We assume that the condition (3.2) holds for $0 \leq t \leq T_{0}$ and that there exists a positive constant $c$ such that for all $0 \leq t \leq T_{0}$ and for all $x \in \mathbf{R}^{d}$, we have $a_{j}(t, x) \geq c$ for $j=1,2$. Set

$$
\begin{aligned}
& M_{j}:=\sup _{t \in\left[0, T_{0}\right]}\left\|\left(\eta_{j}, \psi_{j}, V_{j}, B_{j}\right)(t)\right\|_{H_{u l}^{s+\frac{1}{2}} \times H_{u l}^{s+\frac{1}{2}} \times H_{u l}^{s} \times H_{u l}^{s}}, \\
& \eta:=\eta_{1}-\eta_{2}, \quad \psi:=\psi_{1}-\psi_{2}, \quad V:=V_{1}-V_{2}, \quad B:=B_{1}-B_{2} .
\end{aligned}
$$

Then we have

$$
\begin{aligned}
& \|(\eta, \psi, V, B)\|_{L^{\infty}\left(\left(0, T_{0}\right), H^{s-\frac{1}{2}} \times H^{s-\frac{1}{2}} \times H^{s-1} \times H^{s-1}\right) u l} \\
& \quad \leq \mathcal{K}\left(M_{1}, M_{2}\right)\left\|\left.(\eta, \psi, V, B)\right|_{t=0}\right\|_{H_{u l}^{s-\frac{1}{2}} \times H_{u l}^{s-\frac{1}{2}} \times H_{u l}^{s-1} \times H_{u l}^{s-1}} .
\end{aligned}
$$

Let

$$
N(T):=\sup _{t \in[0, T]}\|(\eta, \psi, V, B)(t)\|_{H_{u l}^{s-\frac{1}{2}} \times H_{u l}^{s-\frac{1}{2}} \times H_{u l}^{s-1} \times H_{u l}^{s-1}} .
$$

Our goal is to prove an estimate of the form

$$
N(T) \leq \mathcal{K}\left(M_{1}, M_{2}\right) N(0)+T \mathcal{K}\left(M_{1}, M_{2}\right) N(T),
$$

for some non-decreasing function $\mathcal{K}$ depending only on $s$ and $d$. Then, by choosing $T$ small enough, this implies $N(T) \leq 2 \mathcal{K}\left(M_{1}, M_{2}\right) N(0)$ for $T_{1}$ smaller than the minimum of $T_{0}$ and $1 / 2 \mathcal{K}\left(M_{1}, M_{2}\right)$, and iterating the estimate between $\left[T_{1}, 2 T_{1}\right], \ldots,\left[T-T_{1}, T_{1}\right]$ implies Theorem 5.1.

Remark 5.2. Notice that we prove a Lipschitz property in weak norms. This is a general fact related to the fact that the flow map of a quasi-linear equation is not expected to be Lipschitz in the highest norms (this means that one does not expect to control the difference $(\eta, \psi, V, B)$ in $\left.L^{\infty}\left(\left[0, T_{0}\right], H^{s+\frac{1}{2}} \times H^{s+\frac{1}{2}} \times H^{s} \times H^{s}\right)_{u l}\right)$.

The proof of Theorem 5.1 follows the same lines as the proof of the similar result [2, Theorem 5.1]. It follows 4 steps: first we prove a Lipschitz estimate for the Dirichlet-Neumann operator. Then we paralinearize the system satisfied by $(\eta, \psi, V, B)$, symmetrize this system, estimate the good unknowns of the symmetrized system and finally estimate $(\eta, \psi, V, B)$. The Lipschitz estimate of the Dirichlet-Neumann operator is the crucial one and we shall give some details. Having established the paradifferential calculus in uniformly local spaces, the other steps are identical mutatis mutandi as in [2] and we shall skip the proofs.

\subsection{Contraction for the Dirichlet-Neumann operator}

In this section the time being fixed we will skip it. 
Lemma 5.3. Assume $s>1+\frac{d}{2}$. Then there exists $\mathcal{F}: \mathbf{R}^{+} \rightarrow \mathbf{R}^{+}$non-decreasing such that for all $\eta_{1}, \eta_{2} \in H_{u l}^{s+\frac{1}{2}}$ and all $f \in H_{u l}^{s}$ we have

$$
\left\|\left(G\left(\eta_{1}\right)-G\left(\eta_{2}\right)\right) f\right\|_{H_{u l}^{s-\frac{3}{2}}} \leq \mathcal{F}\left(\left\|\left(\eta_{1}, \eta_{2}, f\right)\right\|_{H_{u l}^{s+\frac{1}{2}} \times H_{u l}^{s+\frac{1}{2}} \times H_{u l}^{s}}\right)\left\|\eta_{1}-\eta_{2}\right\|_{H_{u l}^{s-\frac{1}{2}}}
$$

where $H_{u l}^{\sigma}=H_{u l}^{\sigma}\left(\mathbf{R}^{d}\right)$.

Proof. We follow closely [2]. As in (3.15), (3.23) we introduce $\rho_{j}, \tilde{u}_{j}, \alpha_{j}, \beta_{j}, \gamma_{j}$ for $j=1$, 2. Then if $\left.\tilde{u}_{j}\right|_{z=0}=f$ we have

$$
G\left(\eta_{j}\right) f=\left.\left(\frac{1+\left|\nabla_{x} \rho_{j}\right|^{2}}{\partial_{z} \rho_{j}} \partial_{z} \tilde{u}_{j}-\nabla_{x} \rho_{j} \cdot \nabla_{x} \tilde{u}_{j}\right)\right|_{z=0} .
$$

We set $\tilde{u}=\tilde{u}_{1}-\tilde{u}_{2}$. Then

$$
\left(\partial_{z}^{2}+\alpha_{1} \Delta_{x}+\beta_{1} \cdot \nabla_{x} \partial_{z}-\gamma_{1} \partial_{z}\right) \tilde{u}=F
$$

where

$$
F=\left\{\left(\alpha_{2}-\alpha_{1}\right) \Delta_{x}+\left(\beta_{2}-\beta_{1}\right) \cdot \nabla_{x} \partial_{z}-\left(\gamma_{2}-\gamma_{1}\right) \partial_{z}\right\} \tilde{u}_{2} .
$$

Since $s>1+\frac{d}{2}$, Lemma 7.5 with $s_{0}=s-2, s_{1}=s-2, s_{2}=s-1, p=2$ gives (with $J=\left(z_{0}, 0\right)$ and $H_{u l}^{\mu}=H_{u l}^{\mu}\left(\mathbf{R}^{d}\right)$ )

$$
\begin{aligned}
\|F\|_{L^{2}\left(J, H^{s-2}\right)_{u l} \leq} & K\left\{\left\|\alpha_{2}-\alpha_{1}\right\|_{L^{2}\left(J, H^{s-1}\right)_{u l}}\left\|\Delta \tilde{u}_{2}\right\|_{L^{\infty}\left(J, H^{s-2}\right)_{u l}}\right. \\
& +\left\|\beta_{2}-\beta_{1}\right\|_{L^{2}\left(J, H^{s-1}\right)_{u l}}\left\|\nabla_{x} \partial_{z} \tilde{u}_{2}\right\|_{L^{\infty}\left(J, H^{s-2}\right)_{u l}} \\
& \left.+\left\|\gamma_{2}-\gamma_{1}\right\|_{L^{2}\left(J, H^{s-2}\right)_{u l}}\left\|\partial_{z} \tilde{u}_{2}\right\|_{L^{\infty}\left(J, H^{s-1}\right)_{u l}}\right\} .
\end{aligned}
$$

Using (3.23) and Lemma 7.5 we can find a non-decreasing function $\mathcal{F}: \mathbf{R}^{+} \rightarrow \mathbf{R}^{+}$such that

$$
\begin{aligned}
& \left\|\alpha_{2}-\alpha_{1}\right\|_{L^{2}\left(J, H^{s-1}\right)_{u l}}+\left\|\beta_{2}-\beta_{1}\right\|_{L^{2}\left(J, H^{s-1}\right)_{u l}}+\left\|\gamma_{2}-\gamma_{1}\right\|_{L^{2}\left(J, H^{s-2}\right)_{u l}} \\
& \quad \leq \mathcal{F}\left(\left\|\left(\eta_{1}, \eta_{2}\right)\right\|_{\left.H_{u l}^{s+\frac{1}{2}} \times H_{u l}^{s+\frac{1}{2}}\right)\left\|\eta_{1}-\eta_{2}\right\|_{H_{u l}}^{s-\frac{1}{2}} .} .\right.
\end{aligned}
$$

On the other hand by Theorem 3.12 with $\sigma=s-1$ we have

$$
\left\|\nabla_{x, z} \tilde{u}_{2}\right\|_{L^{\infty}\left(\left(z_{0}, 0\right), H^{s-1}\right)_{u l}} \leq \mathcal{F}\left(\left\|\eta_{2}\right\|_{H_{u l}^{s+\frac{1}{2}}}\right)\|f\|_{H_{u l}^{s}} .
$$

Combining these estimates we obtain eventually

$$
\|F\|_{L^{2}\left(J, H^{s-2}\right)_{u l}} \leq \mathcal{F}\left(\left\|\left(\eta_{1}, \eta_{2}, f\right)\right\|_{H_{u l}^{s+\frac{1}{2}} \times H_{u l}^{s+\frac{1}{2}} \times H_{u l}^{s}}\right)\left\|\eta_{1}-\eta_{2}\right\|_{H_{u l}^{s-\frac{1}{2}}} .
$$

Since $\tilde{u}$ vanishes at $z=0$ Theorem 3.12 with $\sigma=s-\frac{3}{2}$ gives

$$
\left\|\nabla_{x, z} \tilde{u}\right\|_{C^{0}\left(\left(z_{0}, 0\right), H^{s-\frac{3}{2}}\right)_{u l}} \leq \mathcal{F}\left(\left\|\left(\eta_{1}, \eta_{2}, f\right)\right\|_{H_{u l}^{s+\frac{1}{2}} \times H_{u l}^{s+\frac{1}{2}} \times H_{u l}^{s}}\right)\left\|\eta_{1}-\eta_{2}\right\|_{H_{u l}^{s-\frac{1}{2}}} .
$$

Using (5.5) and Proposition 7.3(i) we obtain (5.4).

\subsection{Paralinearization of the equations}

Notice that it is enough to estimate $\eta, B, V$. Indeed, since $V_{j}=\nabla_{x} \psi_{j}-B_{j} \nabla_{x} \eta_{j}$, one can estimate the $L^{\infty}\left([0, T], H^{s-\frac{3}{2}}\right)_{u l}$-norm of $\nabla_{x} \psi$ from the identity

$$
\nabla_{x} \psi=V+B \nabla_{x} \eta_{1}+B_{2} \nabla_{x} \eta
$$


Lemma 5.4. (See [2, Lemma 5.6].) The differences $\zeta, B, V$ satisfy a system of the form

$$
\left\{\begin{array}{l}
\left(\partial_{t}+V_{1} \cdot \nabla\right)\left(V+\zeta_{1} B\right)+a_{2} \zeta=f_{1}, \\
\left(\partial_{t}+V_{2} \cdot \nabla\right) \zeta-G\left(\eta_{1}\right) V-\zeta_{1} G\left(\eta_{1}\right) B=f_{2},
\end{array}\right.
$$

for some remainders such that

$$
\left\|\left(f_{1}, f_{2}\right)\right\|_{L^{\infty}\left([0, T], H^{s-1} \times H^{s-\frac{3}{2}}\right)_{u l}} \leq \mathcal{K}\left(M_{1}, M_{2}\right) N(T) .
$$

\subsection{Estimates for the good unknown}

In this section we introduce the good-unknown of Alinhac in $[4,1,5,6]$ and symmetrize the system. Let $I=[0, T]$.

Lemma 5.5. (See [2, Lemma 5.7].) Set

$$
\ell:=\sqrt{\lambda_{1} a_{2}}, \quad \varphi:=T_{\sqrt{\lambda_{1}}}\left(V+\zeta_{1} B\right), \quad \vartheta:=T_{\sqrt{a_{2}}} \zeta .
$$

Then

$$
\begin{aligned}
& \left(\partial_{t}+T_{V_{1}} \cdot \nabla\right) \varphi+T_{\ell} \vartheta=g_{1}, \\
& \left(\partial_{t}+T_{V_{2}} \cdot \nabla\right) \vartheta-T_{\ell} \varphi=g_{2},
\end{aligned}
$$

where

$$
\left\|\left(g_{1}, g_{2}\right)\right\|_{L^{\infty}\left(I, H^{s-\frac{3}{2}} \times H^{s-\frac{3}{2}}\right)_{u l}} \leq \mathcal{K}\left(M_{1}, M_{2}\right) N(T) .
$$

Once this symmetrization has been performed, simple energy estimates allow to prove

Lemma 5.6. (See [2, Lemma 5.8].) Let

$$
N^{\prime}(T):=\sup _{t \in I}\left\{\|\vartheta(t)\|_{H_{u l}^{s-\frac{3}{2}}}+\|\varphi(t)\|_{H_{u l}^{s-\frac{3}{2}}}\right\} .
$$

We have

$$
N^{\prime}(T) \leq \mathcal{K}\left(M_{1}, M_{2}\right)(N(0)+T N(T)) .
$$

\subsection{Back to the original unknowns}

From the estimates in Lemma 5.6, it is fairly easy to recover estimates for $\eta$.

Lemma 5.7. (See [2, Lemma 5.9].)

$$
\|\eta\|_{L^{\infty}\left(I ; H^{s-\frac{1}{2}}\right)_{u l}} \leq \mathcal{K}\left(M_{1}, M_{2}\right)\{N(0)+T N(T)\} .
$$

We now estimate $(V, B)$

Proposition 5.8. (Se [2, Proposition 5.10].)

$$
\|(V, B)\|_{L^{\infty}\left(I, H^{s-1} \times H^{s-1}\right)_{u l}} \leq \mathcal{K}\left(M_{1}, M_{2}\right)\{N(0)+T N(T)\} .
$$

The proof will require several preliminary lemmas. We begin by noticing that it is enough to estimate $B$. Indeed, if

$$
\|B\|_{L^{\infty}\left(I, H^{s-1}\right)_{u l}} \leq \mathcal{K}\left(M_{1}, M_{2}\right)\{N(0)+T N(T)\},
$$

then, the estimate of $\varphi$ in (5.9) above allows to recover an estimate for $V+\zeta_{1} B$ (by applying $T_{\sqrt{\lambda_{1}}}-1$ ), which in turn implies the estimate for $V$. 
Let $v=\tilde{\phi}_{1}-\tilde{\phi}_{2}$, where $\tilde{\phi}_{j}$ is the harmonic extension in $\widetilde{\Omega}$ of the function $\psi_{j}$ and set

$$
b_{2}:=\frac{\partial_{z} \tilde{\phi}_{2}}{\partial_{z} \rho_{2}}, \quad w=v-T_{b_{2}} \rho .
$$

We have

$$
\left.w\right|_{z=0}=\psi-T_{B_{2}} \eta
$$

We first state the following result.

Lemma 5.9. (See [2, Lemma 5.11].) We have

$$
\left\|\psi-T_{B_{2}} \eta\right\|_{L^{\infty}\left(I, H^{s}\right)_{u l}} \leq \mathcal{K}\left(M_{1}, M_{2}\right)\{N(0)+T N(T)\} .
$$

We next relate $w, \rho$ and $B$.

Lemma 5.10. (See [2, Lemma 5.12].) We have

$$
B=\left.\left[\frac{1}{\partial_{z} \rho_{1}}\left(\partial_{z} w-\left(b_{2}-T_{b_{2}}\right) \partial_{z} \rho+T_{\partial_{z} b_{2}} \rho\right)\right]\right|_{z=0} .
$$

Lemma 5.11. (See [2, Lemma 5.13].) Recall that $b_{2}:=\frac{\partial_{z} \tilde{\phi}_{2}}{\partial_{z} \rho_{2}}$. For $k=0,1,2$, we have

$$
\left\|\partial_{z}^{k} b_{2}\right\|_{C^{0}\left([-1,0], L^{\infty}\left(I, H^{s-\frac{1}{2}-k}\right)_{u l}\right)} \leq C\left\|\psi_{2}\right\|_{L^{\infty}\left(H^{s+\frac{1}{2}}\right)_{u l}}
$$

for some constant $C$ depending only on $\left\|\eta_{2}\right\|_{L^{\infty}\left(H^{s+\frac{1}{2}}\right)_{u l}}$.

Notice that $\eta$ and hence $\rho$ are estimated in $L^{\infty}\left(I ; H^{s-\frac{1}{2}}\right.$ ) (see (5.10)). To complete the proof of Proposition 5.8, it remains only to estimate $\left.\partial_{z} w\right|_{z=0}$ in $L^{\infty}\left(I, H_{u l}^{s-1}\right)$.

Lemma 5.12. (See [2, Lemma 5.14].) For $t \in[0, T]$ we have

$$
\left\|\nabla_{x, z} w\right\|_{C^{0}\left([-1,0], H^{s-1}\right)_{u l}} \leq \mathcal{K}\left(M_{1}, M_{2}\right)\{N(0)+T N(T)\} .
$$

\subsection{Well posedness}

The proof goes as follows. In a first step we prove the main theorem for very smooth data, using a parabolic regularization. Then, when the data are rough, we regularize them, thus obtaining a sequence of solutions living on an interval depending on a small parameter $\varepsilon$. In a second step, using the tame estimates proved in Proposition 4.7, we show that this sequence exists on a fixed interval. In the last step, using the results stated in Section 7, we prove that it is a Cauchy sequence and we conclude. Let us notice that most of this work has been already done in [2] in the case of the classical Sobolev spaces. Therefore we will only sketch here the main points.

\subsection{Parabolic regularization}

We assume first that $\left(\eta_{0}, \psi_{0}\right) \in H_{u l}^{s} \times H_{u l}^{s}$ for $s \geq n_{0}+\frac{d}{2}, n_{0}$ large enough, and we consider for $\varepsilon>0$ the problem

$$
\left\{\begin{array}{l}
\partial_{t} \eta=G(\eta) \psi+\varepsilon \Delta_{x} \eta, \\
\partial_{t} \psi=-\frac{1}{2}\left|\nabla_{x} \psi\right|^{2}+\frac{1}{2} \frac{\left(\nabla_{x} \eta \cdot \nabla_{x} \psi+G(\eta) \psi\right)^{2}}{1+\left|\nabla_{x} \eta\right|^{2}}-g \eta+\varepsilon \Delta_{x} \psi \\
\left.(\eta, \psi)\right|_{t=0}=\left(\eta_{0}, \psi_{0}\right) .
\end{array}\right.
$$


Setting $U=(\eta, \psi)$ we can rewrite this problem as

$$
U(t)=e^{\varepsilon t \Delta_{x}} U_{0}+\int_{0}^{t} e^{\varepsilon(t-\tau) \Delta_{x}}[\mathcal{A}(U(\tau))] d \tau .
$$

We set $I=[0, T]$ and we introduce the space

$$
E_{s}=L^{\infty}\left(I, H^{s}\right)_{u l} \cap L^{2}\left(I, H^{s+1}\right)_{u l} .
$$

According to Lemma 7.10 we have $\left\|e^{\varepsilon t \Delta_{x}} U_{0}\right\|_{E_{s}} \leq C_{\varepsilon}\left\|U_{0}\right\|_{H_{u l}^{s}}=: R$. Then using the estimates

$$
\begin{aligned}
& \|\mathcal{A}(U)\|_{L^{2}\left(I, H^{s}\right)_{u l}} \leq \mathcal{F}\left(\|U\|_{L^{\infty}\left(I, H^{s}\right)_{u l}}\right)\|U\|_{L^{2}\left(I, H^{s+1}\right)_{u l}} \\
& \left\|\mathcal{A}\left(U_{1}\right)-\mathcal{A}\left(U_{2}\right)\right\|_{L^{2}\left(I, H^{s}\right)_{u l}} \leq \mathcal{F}\left(\left\|\left(U_{1}, U_{2}\right)\right\|_{L^{\infty}\left(I, H^{s} \times H^{s}\right)_{u l}}\right)\left\|U_{1}-U_{2}\right\|_{L^{2}\left(I, H^{s+1}\right)_{u l}}
\end{aligned}
$$

we can show that, if $T=T_{\varepsilon}$ is small enough, the right hand side of (5.16) maps the ball of radius $2 R$ in $E_{s}$ into itself and is contracting. By the Banach principle Eq. (5.16) has a maximal solution on $\left[0, T_{\varepsilon}^{*}\right)$. Moreover if $T_{\varepsilon}<+\infty$ then

$$
\lim _{t \rightarrow T_{\varepsilon}^{*}}\|(\eta, \psi)(t)\|_{H_{u l}^{s} \times H_{u l}^{s}}=+\infty .
$$

Now with this large $s$ we set

$$
M_{s}^{\varepsilon}(T)=\sup _{t \in[0, T]}\left\|\left(\eta^{\varepsilon}, \psi^{\varepsilon}, V^{\varepsilon}, B^{\varepsilon}\right)(t)\right\|_{H_{u l}^{s} \times H_{u l}^{s} \times H_{u l}^{s-1} \times H_{u l}^{s-1} .}
$$

Using the same computations as in [2] and the method of proof of Proposition 4.7 (but in an easier way since here $s$ is large) we deduce that one can find $\mathcal{F}: \mathbf{R}^{+} \rightarrow \mathbf{R}^{+}$strictly increasing such that

$$
M_{s}^{\varepsilon}(T) \leq \mathcal{F}\left(M_{s}^{\varepsilon}(0)+\sqrt{T} M_{s}^{\varepsilon}(T)\right) .
$$

Since $M_{s}^{\varepsilon}(0)=M_{S}(0)$ does not depend on $\varepsilon$, this will imply that there exists $T_{0}>0$ independent of $\varepsilon$ such that $M_{s}^{\varepsilon}(T) \leq \mathcal{F}\left(2 M_{s}(0)\right)$ for $T \in\left[0, T_{0}\right]$. Using this uniform bound on this fixed interval and the arguments of [2] we can pass to the limit in Eqs. (5.15) to obtain a solution $(\eta, \psi)$ of the water wave system.

\subsection{Regularizing the data, a priori estimates}

Assume $\left(\eta_{0}, \psi_{0}, V_{0}, B_{0}\right)$ belongs to $H_{u l}^{s_{0}+\frac{1}{2}} \times H_{u l}^{s_{0}+\frac{1}{2}} \times H_{u l}^{s_{0}} \times H_{u l}^{s_{0}}$ where $s_{0}>1+\frac{d}{2}$. Let $j \in C_{0}^{\infty}\left(\mathbf{R}^{d}\right), j(\xi)=1$ when $|\xi| \leq 1$. We regularize the data in setting $f_{0}^{\varepsilon}=j(\varepsilon D) f_{0}$ if $f_{0}$ is one of them. Then the regularized data belong to $H_{u l}^{s}$ for $s$ large. Therefore applying Step 1 we get a very regular solution $U_{\varepsilon}=\left(\eta_{\varepsilon}, \psi_{\varepsilon}, V_{\varepsilon}, B_{\varepsilon}\right)$ of the water wave system, on an interval $\left[0, T_{\varepsilon}\right]$ which satisfies $M_{S}^{\varepsilon}\left(T_{\varepsilon}\right)<+\infty$ and $\eta_{\varepsilon}(t, x)-\eta_{*}(x)>\frac{h}{2}$ for all $t \in\left[0, T_{\varepsilon}\right], x \in \mathbf{R}^{d}$.

Let $T_{\varepsilon}^{*}>0$ be the supremum of such $T_{\varepsilon}$.

If $T_{\varepsilon}^{*}<+\infty$ then either

$$
\left\{\begin{array}{l}
\lim _{T \rightarrow T_{\varepsilon}^{*}} M_{S}^{\varepsilon}(T)=+\infty \quad \text { or } \\
\lim _{T \rightarrow T_{\varepsilon}^{*}} \eta_{\varepsilon}(t, x)-\eta_{*}(x)=\frac{h}{2}, \quad \text { for some } x \in \mathbf{R}^{d} .
\end{array}\right.
$$

Recall (see Theorem 2.3) that $\eta^{0}(x)-\eta_{*}(x) \geq 2 h$ for all $x \in \mathbf{R}^{d}$. From now on we consider $\varepsilon>0$ so small that

$$
\eta_{\varepsilon}^{0}(x)-\eta_{*}(x) \geq \frac{3 h}{2}, \quad \forall x \in \mathbf{R}^{d} .
$$

We first apply Proposition 4.7 with $s=s_{0}$ and we obtain

$$
M_{s_{0}}^{\varepsilon}(T) \leq \mathcal{F}_{1}\left(M_{s_{0}}^{\varepsilon}(0)+\sqrt{T} M_{s_{0}}^{\varepsilon}(T)\right) .
$$

Since there exists $A_{0}>0$, independent of $\varepsilon$, such that $M_{s_{0}}^{\varepsilon}(0) \leq A_{0}$, for all $\varepsilon>0$ small, we deduce that one can find $T_{0}>0$ independent of $\varepsilon$ such that $M_{s_{0}}^{\varepsilon}(T) \leq \mathcal{F}_{1}\left(2 A_{0}\right)$ for all $T \leq \min \left(T_{0}, T_{\varepsilon}^{*}\right)$. We apply again Proposition 4.7 with 
$s$ large and we get

$$
M_{s}^{\varepsilon}(T) \leq \mathcal{F}\left(A_{0}+\sqrt{T_{0}} \mathcal{F}_{1}\left(2 A_{0}\right)\right)\left(M_{s}^{\varepsilon}(0)+\sqrt{T} M_{s}^{\varepsilon}(T)\right) .
$$

Let $0<T_{1} \leq T_{0}$ be such that $\sqrt{T_{1}} \mathcal{F}\left(A_{0}+\sqrt{T_{0}} \mathcal{F}_{1}\left(2 A_{0}\right)\right) \leq \frac{1}{2}$. Then

$$
M_{s}^{\varepsilon}(T) \leq 2 \mathcal{F}\left(A_{0}+\sqrt{T_{0}} \mathcal{F}_{1}\left(2 A_{0}\right)\right) A_{0}=: K_{0}, \quad T \leq \min \left(T_{1}, T_{\varepsilon}^{*}\right) .
$$

Now on $[0, T]$ we can write

$$
\eta_{\varepsilon}(t, x)-\eta_{\varepsilon}^{0}(x)=\int_{0}^{t} \partial_{t} \eta_{\varepsilon}(s, x) d s=\int_{0}^{t}\left(G\left(\eta_{\varepsilon}\right) \psi_{\varepsilon}\right)(s, x) d s
$$

from which we deduce, since $s$ is large

$$
\begin{aligned}
\left\|\eta_{\varepsilon}(t, \cdot)-\eta_{\varepsilon}^{0}(\cdot)\right\|_{L^{\infty}\left(\mathbf{R}^{d}\right)} & \leq T \sup _{t \in[0, T]}\left\|\left(G\left(\eta_{\varepsilon}\right) \psi_{\varepsilon}\right)(t, \cdot)\right\|_{H^{s-2}\left(\mathbf{R}^{d}\right)} \\
& \leq T \mathcal{F}_{2}\left(M_{s}^{\varepsilon}(T)\right) \leq T \mathcal{F}_{2}\left(K_{0}\right) .
\end{aligned}
$$

Let $0<T_{2} \leq T_{1}$ be such that $T_{2} \mathcal{F}_{2}\left(K_{0}\right) \leq \frac{h}{10}$. It follows that

$$
\eta_{\varepsilon}(t, x)-\eta_{*}(x) \geq h, \quad \forall t \in\left[0, \min \left(T_{2}, T_{\varepsilon}^{*}\right)\right), \quad \forall x \in \mathbf{R}^{d} .
$$

This implies that $T_{\varepsilon}^{*} \geq T_{2}$ for all small $\varepsilon$. Indeed if there exists an $\varepsilon_{0}$ such that $T_{\varepsilon_{0}}^{*} \leq T_{2}$ then we would have (5.21) and (5.22) on $\left[0, T_{\varepsilon_{0}}^{*}\right)$ which would contradict (5.19).

This shows that our solution $\left(\eta_{\varepsilon}, \psi_{\varepsilon}, V_{\varepsilon}, B_{\varepsilon}\right)$ exists on a fixed interval $\left[0, T_{2}\right]$. Moreover, as seen above, $M_{S}^{\varepsilon}(T)$ is uniformly bounded on this interval.

\subsection{Passing to the limit}

According to Theorem 5.1, $\left(\eta_{\varepsilon}, \psi_{\varepsilon}, V_{\varepsilon}, B_{\varepsilon}\right)$ which is, according to Section 5.8, bounded in

$$
L^{\infty}\left((0, T) ; H_{u l}^{s_{0}+\frac{1}{2}} \times H_{u l}^{s_{0}+\frac{1}{2}} \times H_{u l}^{s_{0}} \times H_{u l}^{s_{0}}\right),
$$

is convergent in

$$
L^{\infty}\left((0, T) ; H_{u l}^{s_{0}-\frac{1}{2}} \times H_{u l}^{s_{0}-\frac{1}{2}} \times H_{u l}^{s_{0}-1} \times H_{u l}^{s_{0}-1}\right),
$$

and hence also for any $\delta>0$ in

$$
L^{\infty}\left((0, T) ; H_{u l}^{s_{0}+\frac{1}{2}-\delta} \times H_{u l}^{s_{0}+\frac{1}{2}-\delta} \times H_{u l}^{s_{0}-\delta} \times H_{u l}^{s_{0}-\delta}\right)
$$

To get the existence of solutions, it remains to pass to the limit in the equations (the uniqueness follows once again from Theorem 5.1). For this step, we rewrite the system (2.1), (2.2) as

$$
\left\{\begin{array}{l}
\partial_{t} \eta_{\varepsilon}=G\left(\eta_{\varepsilon}\right) \psi_{\varepsilon} \\
\partial_{t} \psi_{\varepsilon}+V_{\varepsilon} \cdot \nabla_{x} \psi_{\varepsilon}=\frac{1}{2}\left(V_{\varepsilon}^{2}+B_{\varepsilon}^{2}\right)-g \eta_{\varepsilon} \\
B_{\varepsilon}=\frac{\nabla_{x} \eta_{\varepsilon} \cdot \nabla_{x} \psi_{\varepsilon}+G\left(\eta_{\varepsilon}\right) \psi_{\varepsilon}}{1+\left|\nabla_{x} \eta_{\varepsilon}\right|^{2}} \\
V_{\varepsilon}=\nabla_{x} \psi_{\varepsilon}-B_{\varepsilon} \nabla_{x} \eta_{\varepsilon}
\end{array}\right.
$$

Choosing $\delta>0$ such that $s-\delta-\frac{1}{2}>\frac{d}{2}$ (so that $H^{s-\delta-\frac{1}{2}}$ is an algebra), we deduce that 


$$
\begin{aligned}
& \partial_{t} \eta_{\varepsilon} \rightarrow \partial_{t} \eta \quad \text { in } \mathcal{D}^{\prime}\left((0, T) \times \mathbf{R}^{d}\right) \\
& \partial_{t} \psi_{\varepsilon} \rightarrow \partial_{t} \psi \quad \text { in } \mathcal{D}^{\prime}\left((0, T) \times \mathbf{R}^{d}\right) \\
& V_{\varepsilon} \cdot \nabla_{x} \psi_{\varepsilon} \rightarrow V \cdot \nabla_{x} \psi \quad \text { in } L^{\infty}\left((0, T) ; H_{u l}^{s-\delta-\frac{1}{2}}\right) \\
& V_{\varepsilon}^{2}+B_{\varepsilon}^{2} \rightarrow V^{2}+B^{2} \quad \text { in } L^{\infty}\left((0, T) ; H_{u l}^{s-\delta}\right) \\
& \nabla_{x} \eta_{\varepsilon} \cdot \nabla_{x} \psi_{\varepsilon} \rightarrow \nabla_{x} \eta \cdot \nabla_{x} \psi \quad \text { in } L^{\infty}\left((0, T) ; H_{u l}^{s-\delta-\frac{1}{2}}\right) \subset L^{\infty}\left((0, T) ; L_{u l}^{2}\right) \\
& \left|\nabla_{x} \eta_{\varepsilon}\right|^{2} \rightarrow\left|\nabla_{x} \eta\right|^{2} \quad \text { in } L^{\infty}\left((0, T) ; H_{u l}^{s-\delta-\frac{1}{2}}\right) \subset L^{\infty}\left((0, T) ; C^{0} \cap L^{\infty}\left(\mathbf{R}^{d}\right)\right)
\end{aligned}
$$

On the other hand, according to Lemma 5.3, we get

$$
G\left(\eta_{\varepsilon}\right) \psi_{\varepsilon}-G(\eta) \psi=G\left(\eta_{\varepsilon}\right)\left(\psi_{\varepsilon}-\psi\right)+\left(G\left(\eta_{\varepsilon}\right)-G(\eta)\right) \psi \rightarrow 0,
$$

in

$$
L^{\infty}\left((0, T) ; H_{u l}^{s-\frac{3}{2}-\delta}\right) \subset L^{\infty}\left((0, T) ; L_{u l}^{2}\right),
$$

which allows to pass to he limit in (5.23) and show that the same system of equations is satisfied by $(\eta, \psi, V, B)$ in $\mathcal{D}^{\prime}\left((0, T) \times \mathbf{R}^{d}\right)$.

\subsection{Continuity in time}

We now prove that $(\eta, \psi, V, B)$ is continuous in time with values in $H_{u l}^{s_{0}+\frac{1}{2}-\delta} \times H_{u l}^{s_{0}+\frac{1}{2}-\delta} \times H_{u l}^{s_{0}-\delta} \times H_{u l}^{s_{0}-\delta}$. From the equation, and product rules, its time derivative is clearly in

$$
L^{\infty}\left((0, T) ; H_{u l}^{s_{0}-\frac{1}{2}} \times H_{u l}^{s_{0}-\frac{1}{2}} \times H_{u l}^{s_{0}-\frac{3}{2}} \times H_{u l}^{s_{0}-\frac{3}{2}}\right)
$$

and consequently (interpolating with the a priori estimate), for any $\delta>0$,

$$
(\eta, \psi, V, B) \in C^{0}\left([0, T) ; H_{u l}^{s_{0}+\frac{1}{2}-\delta} \times H_{u l}^{s_{0}+\frac{1}{2}-\delta} \times H_{u l}^{s_{0}-\delta} \times H_{u l}^{s_{0}-\delta}\right) .
$$

\section{The canal}

We consider now the case of a canal having vertical walls near the free surface or the case of a rectangular basin.

The propagation of waves whose crests are orthogonal to the walls is one of the main motivation for the analysis of 2D waves. It was historically at the heart of the analysis of water waves. The study of the propagation of three-dimensional water waves for the linearized equations goes back to Boussinesq (see [9]). However, there are no existence results for the nonlinear equations in the general case where the waves can be reflected on the walls of the canals (except the analysis of 3D-periodic traveling waves which correspond to the reflexion of a 2D-wave off a vertical wall, see Reeder and Shinbrot [29], Craig and Nicholls [13] and Iooss and Plotnikov [21]).

We hence consider a fluid domain which at time $t$ is of the form

$$
\Omega(t)=\left\{\left(x_{1}, x_{2}, y\right) \in M \times \mathbf{R}: b(x)<y<\eta(t, x), x=\left(x_{1}, x_{2}\right)\right\},
$$

where $M=(0,1) \times \mathbf{R}$ in the case of the canal and $M=(0,1) \times(0, L)$ in the case of a rectangular basin, and $b$ is a fixed continuous function on $M$ describing the bottom.

Denote by $\Sigma$ the free surface and by $\Gamma$ the fixed boundary of the canal:

$$
\Sigma(t)=\left\{\left(x_{1}, x_{2}, y\right) \in M \times \mathbf{R}: y=\eta(t, x)\right\},
$$

and we set $\Gamma=\partial \Omega(t) \backslash \Sigma(t)$ (which does not depend on time). We have

$$
\begin{aligned}
& \Gamma=\Gamma_{1} \cup \Gamma_{2}, \\
& \Gamma_{1}=\left\{\left(x_{1}, x_{2}, y\right) \in M \times \mathbf{R} ; b(x)=y\right\} \\
& \Gamma_{2}=\left\{\left(x_{1}, x_{2}, y\right) \in \partial M \times \mathbf{R} ; b(x)<y<\eta\left(x_{1}, x^{\prime}\right)\right\} .
\end{aligned}
$$




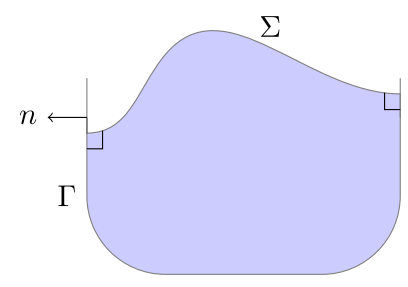

Fig. 1. Two-dimensional section of the fluid domain, exhibiting the right-angles at the interface $\Sigma \cap \Gamma$.

Denote by $n$ the normal to the boundary $\Gamma$ and denote by $v$ the normal to the free surface $\Sigma$. The irrotational water-waves system is then the following: the Eulerian velocity field $v: \Omega \rightarrow \mathbf{R}^{3}$ solves the incompressible Euler equation

$$
\partial_{t} v+v \cdot \nabla_{x, y} v+\nabla_{x, y} P=-g e_{y}, \quad \operatorname{div}_{x, y} v=0, \quad \operatorname{curl}_{x, y} v=0 \quad \text { in } \Omega,
$$

where $-g e_{y}$ is the acceleration of gravity $(g>0)$ and where the pressure term $P$ can be recovered from the velocity by solving an elliptic equation. The problem is then given by three boundary conditions. They are

$$
\begin{cases}v \cdot n=0 & \text { on } \Gamma, \\ \partial_{t} \eta=\sqrt{1+|\nabla \eta|^{2}} v \cdot v & \text { on } \Sigma, \\ P=0 & \text { on } \Sigma .\end{cases}
$$

We notice that the first condition in (6.3) expresses the fact that the particles in contact with the rigid bottom remain in contact with it. Notice that to fully make sense, this condition requires some smoothness on $\Gamma$, but in general it has a weak variational meaning (see Section 3).

Finally we impose the initial condition

$$
\left.(\eta, v)\right|_{t=0}=\left(\eta_{0}, v_{0}\right),
$$

where $v_{0}$ satisfies

$$
\operatorname{div}_{x, y} v_{0}=0, \quad \operatorname{curl}_{x, y} v_{0}=0 \quad \text { in } \Omega_{0}, \quad v_{0} \cdot n=0, \quad \text { on } \Gamma .
$$

It follows that there exists a function $\phi_{0}: \Omega_{0} \rightarrow \mathbf{R}$ such that

$$
v_{0}=\nabla_{x, y} \phi_{0} \quad \text { in } \Omega_{0}, \text { with } \Delta_{x, y} \phi_{0}=0 .
$$

We set

$$
\psi_{0}=\left.\phi_{0}\right|_{y=\eta_{0}(x)}
$$

and introduce the trace of the velocity field $v_{0}=\left(v_{0, x_{1}}, v_{0, x_{2}}, v_{0, y}\right)$ on $\Sigma_{0}=\left\{\left(x, \eta_{0}(x)\right)\right\}$ in setting

$$
\left.v_{0, x_{1}}\right|_{y=\eta_{0}}=V_{0, x_{1}},\left.\quad v_{0, x_{2}}\right|_{y=\eta_{0}}=V_{0, x_{2}},\left.\quad v_{0, y}\right|_{y=\eta_{0}}=B_{0}, \quad V_{0}=\left(V_{0, x_{1}}, V_{0, x_{2}}\right) .
$$

Similarly, to a solution $v$ of (6.2)-(6.3) we associate $\phi, \psi$ and $(V, B)=\left.v\right|_{y=\eta}$ as above.

The stability of the waves is dictated by the Taylor sign condition, which is the assumption that there exists a positive constant $c$ such that

$$
a(t, x):=-\left(\partial_{y} P\right)(t, x, \eta(t, x)) \geq c>0 .
$$

\subsection{A simple observation}

We begin with an elementary calculation showing that, at least for regular enough solutions, as soon as the Taylor sign condition (6.5) is satisfied, in the case of vertical walls, it is necessary that at the points where the free surface and the boundary of the canal meet $(\Sigma(t) \cap \Gamma)$, the scalar product between the two normals (to the free surface and to the boundary of the canal) vanishes: $v \cdot n=0$ on $\Sigma \cap \Gamma$, which means that the free surface $\Sigma$ necessarily makes a right-angle with the rigid walls (see Fig. 1). 
Proposition 6.1. Let $(\eta, v)$ be a classical solution of system (6.2), (6.3) such that the Taylor coefficient a is continuous and non-vanishing and $\eta(t, x) \geq b(x)+h$ for some positive constant $h$. Then the angle between the free surface, $\Sigma(t)$ and the boundary of the canal $\Gamma$ is a right angle:

$$
\forall t \in[0, T], \forall x \in \Sigma(t) \cap \Gamma, \quad n \cdot v(t, x)=0,
$$

which is equivalent to

$$
\left.\partial_{x_{1}} \eta\left(t, x_{1}, x_{2}\right)\right|_{x_{1}=0,1}=0 .
$$

Remark 6.2. Notice that here, to get the right angle property, we assume that the Taylor coefficient does not vanish. It could happen that this is not the case, in which case, this right angle property could be violated.

Proof of Proposition 6.1. We give the proof in the case of a canal, the proof for the rectangular basin is similar. Since $\eta_{0}(x) \geq b(x)+h$ at a point $m_{0}$ where $\Sigma(t)$ and $\Gamma$ meet we have $m_{0}=\left(\varepsilon, x_{2}^{0}, y^{0}\right)$ where $\varepsilon=0$ or 1 . Let $m=\left(\varepsilon, x_{2}, y\right)$ be a point on $\Gamma$ near $m_{0}$. At $m$ the normal $n$ to $\Gamma$ is $n=( \pm 1,0,0)$. Taking the scalar product of Eq. (6.2) with $n$ we obtain, since $e_{y} \cdot n=0$,

$$
\left(\nabla_{x, y} P\right) \cdot n=-\left(\partial_{t} v\right) \cdot n-\left(\left(v \cdot \nabla_{x, y}\right) v\right) \cdot n \quad \text { at } m .
$$

Denote by $\left(v_{x_{1}}, v_{x_{2}}, v_{y}\right)$ the three components of the velocity field $v$. The first condition in (6.3) implies that $(v \cdot n)(m)= \pm v_{x_{1}}\left(t, \varepsilon, x_{2}, y\right)=0$. It follows that $\left(\partial_{t} v\right) \cdot n=\partial_{t}(v \cdot n)=0$ at $m$. Moreover on $\Gamma$ near $m_{0}$ we have

$$
\begin{aligned}
{\left[\left(\left(v \cdot \nabla_{x, y}\right) v\right) \cdot n\right]\left(t, \varepsilon, x_{2}, y\right) } & = \pm\left[\left(v \cdot \nabla_{x, y}\right) v_{x_{1}}\right]\left(t, \varepsilon, x_{2}, y\right) \\
& = \pm\left[\left(v_{x_{2}} \partial_{x_{2}}+v_{y} \partial_{y}\right) v_{x_{1}}\right]\left(t, \varepsilon, x_{2}, y\right) \\
& = \pm\left[\left(v_{x_{2}} \partial_{x_{2}}+v_{y} \partial_{y}\right)\right]\left(v_{x_{1}}\left(t, \varepsilon, x_{2}, y\right)\right)=0 .
\end{aligned}
$$

It follows from (6.7) that

$$
\left(\nabla_{x, y} P\right) \cdot n=0 \quad \text { at } m .
$$

Now by the third condition in (6.3) we have $P=0$ on $\Sigma$ and by (6.5) and our hypothesis on the Taylor coefficient we have $\nabla_{x, y} P \neq 0$ on $\Sigma$. It follows that $\nabla_{x, y} P$ is proportional to the normal $v$ at $\Sigma$ and by continuity at $\Sigma \cap \Gamma$. We deduce from (6.8) that $v \cdot n=0$ at $m_{0}$.

Once this right angle property is ensured, it is easy to show that some additional compatibility conditions have also to be fulfilled. Namely, for $f=B_{0}, V_{0, x_{2}}, \partial_{x_{1}} V_{0, x_{1}}$, using (6.3), as soon as the function $\phi$ is smooth enough so that all terms below are defined, we have with $m=\left(\varepsilon, x_{2}\right)(\varepsilon=0$ or 1$)$ :

$$
\left\{\begin{aligned}
\partial_{x_{1}} \psi_{0}(m)= & \partial_{x_{1}} \phi_{0}\left(m, \eta_{0}(m)\right)+\partial_{y} \phi_{0}\left(m, \eta_{0}(m)\right) \partial_{x_{1}} \eta_{0}(m)=0 \\
\partial_{x_{1}} B_{0}(m)= & \partial_{x_{1}} \partial_{y} \phi_{0}\left(m, \eta_{0}(m)\right)+\partial_{y}^{2} \phi_{0}\left(m, \eta_{0}(m)\right) \partial_{x_{1}} \eta_{0}(m)=0, \\
\partial_{x_{1}} V_{0, x_{2}}(m)= & \partial_{x_{1}} \partial_{x_{2}} \phi_{0}\left(m, \eta_{0}(m)\right)+\partial_{y} \partial_{x_{2}} \phi_{0}\left(m, \eta_{0}(m)\right) \partial_{x_{1}} \eta_{0}(m)=0, \\
\partial_{x_{1}}^{2} V_{0, x_{1}}(m)= & \partial_{x_{1}}^{3} \phi_{0}\left(m, \eta_{0}(m)\right)+2 \partial_{y} \partial_{x_{1}}^{2} \phi_{0}\left(m, \eta_{0}(m)\right) \partial_{x_{1}} \eta_{0}(m) \\
& +\partial_{y}^{2} \partial_{x_{1}} \phi_{0}\left(m, \eta_{0}(m)\right)\left(\partial_{x_{1}} \eta_{0}(m)\right)^{2}+\partial_{y} \partial_{x_{1}} \phi_{0}\left(m, \eta_{0}(m)\right) \partial_{x_{1}}^{2} \eta_{0}(m) \\
= & 0,
\end{aligned}\right.
$$

where in the last equality, we used that $\partial_{x_{1}}^{3} \phi=-\left(\partial_{x_{2}}^{2}+\partial_{y}^{2}\right) \partial_{x_{1}} \phi$, since $\phi$ is harmonic.

\subsection{The result}

As before, we denote by $x_{1}$ (resp. $\left.x_{2}\right)$ the variable in $(0,1)$ (resp. in $\mathbf{R}$ ). To state our results we need to introduce the uniformly local Sobolev spaces in the $x_{2}$ direction (these spaces are introduced by Kato in [22]). Let 
$1=\sum_{k \in \mathbf{Z}} \chi\left(x_{2}-k\right)$ be a partition of unity and define for any $s \in \mathbf{R}$,

$$
H_{u l}^{s}((0,1) \times \mathbf{R})=\left\{u \in H_{l o c}^{s}((0,1) \times \mathbf{R}): \sup _{k}\left\|\chi\left(x_{2}-k\right) u\right\|_{H^{s}((0,1) \times \mathbf{R})}<+\infty\right\} .
$$

These are Banach spaces when endowed with the norm

$$
\|u\|_{H_{u l}^{s}}=\sup _{k}\left\|\chi\left(x_{2}-k\right) u\right\|_{H^{s}((0,1) \times \mathbf{R})} .
$$

In Section 6.1 we showed that in order to get smooth solutions, a set of compatibility conditions (6.6), (6.9) have to be assumed. Here we prove that these conditions are not only necessary, but they are sufficient.

Theorem 6.3. Set $M=(0,1) \times \mathbf{R}$. Let $s \in(2,3), s \neq \frac{5}{2}$, and

$$
\mathcal{H}^{s}(M)=H_{u l}^{s+\frac{1}{2}}(M) \times H_{u l}^{s+\frac{1}{2}}(M) \times H_{u l}^{s}(M) \times H_{u l}^{s}(M) .
$$

Consider $\left(\eta_{0}, \psi_{0}, V_{0}, B_{0}\right) \in \mathcal{H}^{s}(M)$ and assume that, with $\varepsilon=0,1$

$\left(H_{1}\right) V_{0, x_{1}}\left(\varepsilon, x_{2}\right)=0$ and $\partial_{x_{1}} f\left(\varepsilon, x_{2}\right)=0$ when $f=\eta_{0}, \psi_{0}, B_{0}, V_{0, x_{2}}$. Furthermore, $\partial_{x_{1}}^{2} V_{0, x_{1}}\left(\varepsilon, x_{2}\right)=0$ if $s>5 / 2$.

$\left(H_{2}\right)$ The Taylor sign condition, $a_{0}(x) \geq c>0$ is satisfied at time $t=0$.

$\left(H_{3}\right) \quad \eta_{0}(x) \geq b(x)+h$ for some positive constant $h$.

Then there exists a time $T>0$ and a unique solution ( $\left.\eta, v=\nabla_{x, y} \phi\right)$ of the system (6.2), (6.3), (6.4) such that

i) $\left(\eta,\left.\phi\right|_{\Sigma}, V, B\right) \in C\left([0, T) ; \mathcal{H}^{s}(M)\right)$,

ii) the Taylor sign condition is satisfied at time $t$ and $\eta(t) \geq b+h / 2$.

In the case of a rectangular basin we have the following result.

Theorem 6.4. Set $M=(0,1) \times(0, L)$. Let $s \in(2,3), s \neq \frac{5}{2}$, and

$$
\mathcal{H}^{s}(M)=H^{s+\frac{1}{2}}(M) \times H^{s+\frac{1}{2}}(M) \times H^{s}(M) \times H^{s}(M) .
$$

Consider initial data $\left(\eta_{0}, \psi_{0}, V_{0}, B_{0}\right) \in \mathcal{H}^{s}(M)$, such that

$\left(C_{1}\right) V_{0, x_{1}}\left(\varepsilon, x_{2}\right)=0$ and $\partial_{x_{1}} f\left(\varepsilon, x_{2}\right)=0$ when $f=\eta_{0}, \psi_{0}, B_{0}, V_{0, x_{2}}$. Furthermore, $\partial_{x_{1}}^{2} V_{0, x_{1}}\left(\varepsilon, x_{2}\right)=0$ if $s>5 / 2$. Here $\varepsilon=0$ or 1 .

$\left(C_{2}\right) V_{0, x_{2}}\left(x_{1}, \delta\right)=0$ and $\partial_{x_{2}} f\left(x_{1}, \delta\right)=0$ when $f=\eta_{0}, \psi_{0}, B_{0}, V_{0, x_{1}}$. Furthermore, $\partial_{x_{2}}^{2} V_{0, x_{2}}\left(x_{1}, \delta\right)=0$ if $s>5 / 2$. Here $\delta=0$ or L.

$\left(C_{3}\right)$ The Taylor sign condition, $a_{0}(x) \geq c>0$ is satisfied at time $t=0$.

$\left(C_{4}\right) \eta_{0}(x) \geq b(x)+h$ for some positive constant $h$.

Then there exists $T>0$ and a unique solution $\left(\eta, v=\nabla_{x, y} \phi\right)$ of (6.2)-(6.4) such that

(1) $\left(\eta,\left.\phi\right|_{\Sigma}, V, B\right) \in C\left([0, T) ; \mathcal{H}^{s}(M)\right)$,

(2) the Taylor sign condition is satisfied at time $t$ and $\eta(t) \geq b+h / 2$.

Remark 6.5. (i) Our results exclude the case $s=\frac{5}{2}$ for technical reasons. It would be possible (but unnecessarily complicated) to include this case.

(ii) In the case of a flat bottom (say $b(x)=-1$ ) we do not need assumption $\left(H_{2}\right)$ (and $\left(C_{3}\right)$ ) which is in this case always satisfied as proved by $\mathrm{Wu}$ [31,30], see also [24]. Also, this condition is satisfied under a smallness assumption.

(iii) Condition $\left(H_{1}\right)$, when $f=\eta_{0}$, says that at $t=0$ the fluid has to be orthogonal to the fixed vertical walls. 


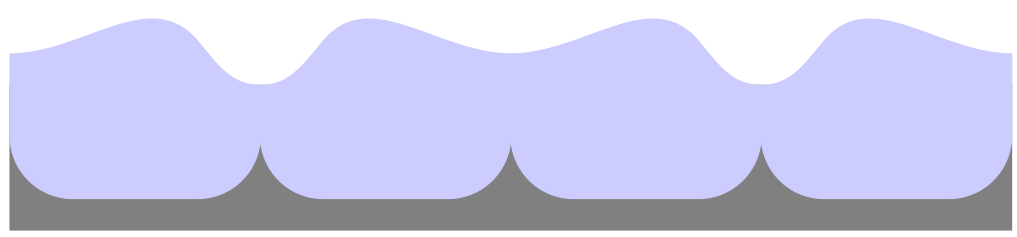

Fig. 2. Two-dimensional section of the extended fluid domain.

\subsection{Proof of the result}

Following Boussinesq (see [9, p. 37]) the strategy of proof is to perform a symmetrization process (following the process which is illustrated on Fig. 2).

Once this symmetrization process is performed, we will apply our result [3, Theorem 2.3] to conclude.

\subsubsection{The periodization process}

Without additional assumptions, the reflection procedure should yield in general a Lipschitz singularity. However, here the possible singularities are weaker according to the physical hypothesis $\left(H_{1}\right)$.

For a function $v$ defined on $(0,+\infty)$, define $v^{\text {ev }}$ and $v^{\text {od }}$ to be the even and odd extensions of $v$ to $(-\infty,+\infty)$ defined by

$$
\begin{aligned}
& v^{\mathrm{ev}}(y)= \begin{cases}v(-y), & \text { if } y<0 \\
v(y) & \text { if } y \geq 0\end{cases} \\
& v^{\mathrm{od}}(y)= \begin{cases}-v(-y), & \text { if } y<0 \\
v(y) & \text { if } y \geq 0 .\end{cases}
\end{aligned}
$$

We have the following result:

\section{Proposition 6.6. We have}

(1) Assume that $0 \leq s<\frac{3}{2}$. Then the map $v \mapsto v^{\mathrm{ev}}$ is continuous from $H^{s}(0,+\infty)$ to $H^{s}(\mathbf{R})$.

(2) Assume that $\frac{3}{2}<s<\frac{7}{2}$. Then the map $v \mapsto v^{\mathrm{ev}}$ is continuous from the space $\left\{v \in H^{s}(0,+\infty): v^{\prime}(0)=0\right\}$ to $H^{s}(\mathbf{R})$.

(3) Assume that $0 \leq s<\frac{1}{2}$. Then the map $v \mapsto v^{\text {od }}$ is continuous from $H^{s}(0,+\infty)$ to $H^{s}(\mathbf{R})$.

(4) Assume that $\frac{1}{2}<s<\frac{5}{2}$. Then the map $v \mapsto v^{\text {od }}$ is continuous from the space $\left\{v \in H^{s}(0,+\infty): v(0)=0\right\}$ to $H^{s}(\mathbf{R})$.

(5) Assume that $\frac{5}{2}<s \leq 4$. Then the map $v \mapsto v^{\text {od }}$ is continuous from the space $\left\{v \in H^{s}(0,+\infty): v(0)=v^{\prime \prime}(0)=0\right\}$ to $H^{s}(\mathbf{R})$.

Proof. Let $I=(0,+\infty)$. Then $C_{0}^{\infty}(\bar{I})$ is dense in $H^{s}(I)$ for all $s \in \mathbf{R}$.

(1) The case $s=0$ is trivial since $\left\|v^{\mathrm{ev}}\right\|_{L^{2}(\mathbf{R})}^{2}=2\|v\|_{L^{2}(I)}^{2}$. Consider now the case $0<s<1$. Then the square of the $H^{s}(\mathbf{R})$-norm of $v^{\text {ev }}$ is equivalent to

$$
\left\|v^{\mathrm{ev}}\right\|_{L^{2}(\mathbf{R})}^{2}+A, \quad A=: \iint_{\mathbf{R} \times \mathbf{R}} \frac{\left|v^{\mathrm{ev}}(x)-v^{\mathrm{ev}}(y)\right|^{2}}{|x-y|^{1+2 s}} d x d y .
$$

Then we can write

$$
A=2 \iint_{I \times I} \frac{|v(x)-v(y)|^{2}}{|x-y|^{1+2 s}} d x d y+2 \iint_{I \times I} \frac{|v(x)-v(y)|^{2}}{|x+y|^{1+2 s}} d x d y:=A_{1}+A_{2} .
$$

We have

$$
A_{1} \leq 2\|v\|_{H^{s}(I)}^{2}, \quad A_{2} \leq 2\|v\|_{H^{s}(I)}^{2}
$$


since $\frac{1}{(x+y)^{1+2 s}} \leq \frac{1}{|x-y|^{1+2 s}}$. The case $s=1$ being straightforward consider the case $1<s<\frac{3}{2}$. Set $\sigma=s-1 \in\left(0, \frac{1}{2}\right)$. Then

$$
\left\|v^{\mathrm{ev}}\right\|_{H^{s}(\mathbf{R})}^{2}=\left\|v^{\mathrm{ev}}\right\|_{L^{2}(\mathbf{R})}^{2}+\left\|\partial_{x} v^{\mathrm{ev}}\right\|_{H^{\sigma}(\mathbf{R})}^{2} .
$$

Since $0<\sigma<1$ we have

$$
\begin{aligned}
& \left\|\partial_{x} v^{\mathrm{ev}}\right\|_{H^{\sigma}(\mathbf{R})}^{2} \leq C\left(A_{0}+A_{1}+A_{2}\right) \\
& A_{0}=\left\|\partial_{x} v^{\mathrm{ev}}\right\|_{L^{2}(\mathbf{R})}^{2} \leq C_{1}\left\|v^{\prime}\right\|_{L^{2}(I)}^{2} \leq C_{1}\|v\|_{H^{s}(I)}^{2} \\
& A_{1}=\iint_{I \times I} \frac{\left|v^{\prime}(x)-v^{\prime}(y)\right|^{2}}{|x-y|^{1+2 \sigma}} d x d y \leq C_{2}\left\|v^{\prime}\right\|_{H^{\sigma}(I)}^{2} \leq C_{2}\|v\|_{H^{s}(I)}^{2} \\
& A_{2}=\iint_{I \times I} \frac{\left|v^{\prime}(x)+v^{\prime}(y)\right|^{2}}{|x+y|^{1+2 \sigma}} d x d y .
\end{aligned}
$$

Eventually we have

$$
A_{2} \leq C_{3} \int_{I} \frac{\left|v^{\prime}(x)\right|^{2}}{|x|^{2 \sigma}} d x \leq C_{4}\left\|v^{\prime}\right\|_{H^{\sigma}(I)} \leq C_{4}\|v\|_{H^{s}(I)}
$$

by Theorem 11.2 in [27], since $0<\sigma<\frac{1}{2}$. This completes the proof of (1).

(2) If $\frac{3}{2}<s<2$ let $\sigma=s-1 \in\left(\frac{1}{2}, 1\right)$; arguing as above we see that

$$
\left\|v^{\mathrm{ev}}\right\|_{H^{s}(\mathbf{R})}^{2} \leq C\left(\|v\|_{H^{s}(I)}^{2}+\int_{I} \frac{\left|v^{\prime}(x)\right|^{2}}{|x|^{2 \sigma}} d x\right) .
$$

Now since $v^{\prime} \in H^{\sigma}(I)$ and $v^{\prime}(0)=0$ we can apply Theorem 11.3 in [27] which ensures that the integral in the right hand side can be estimated by $C\|v\|_{H^{s}(I)}^{2}$. The case $s=2$ being straightforward let $2<s \leq \frac{7}{2}$. Then

$$
\left\|v^{\mathrm{ev}}\right\|_{H^{s}(\mathbf{R})}^{2} \leq C\left(\left\|v^{\mathrm{ev}}\right\|_{L^{2}(\mathbf{R})}^{2}+\left\|\partial_{x}^{2} v^{\mathrm{ev}}\right\|_{H^{s-2}(\mathbf{R})}^{2}\right) .
$$

Since $0<s-2<\frac{3}{2}$ and $v^{\prime}(0)=0$ we may apply the same argument as in the case (1) to ensure that $\left\|\partial_{x}^{2} v^{\mathrm{ev}}\right\|_{H^{s-2}(\mathbf{R})}^{2} \leq$ $C\|v\|_{H^{s}(I)}^{2}$.

The cases (3) to (5) are proved by exactly the same arguments.

To state the reflection procedure in higher dimension we need to introduce the uniformly local Sobolev spaces in $\mathbf{R}^{n}, n \geq 2$.

Let $1=\sum_{k \in \mathbf{Z}^{n}} \chi(x-k)$ be a partition of unity in $\mathbf{R}^{n}$ and define for any $s \in \mathbf{R}$,

$$
H_{u l}^{s}\left(\mathbf{R}^{n}\right)=\left\{u \in H_{l o c}^{s}\left(\mathbf{R}^{n}\right): \sup _{k}\|\chi(\cdot-k) u\|_{H^{s}\left(\mathbf{R}^{n}\right)}<+\infty\right\} .
$$

These are Banach spaces when endowed with the norm

$$
\|u\|_{H_{u l}^{s}}=\sup _{k}\|\chi(\cdot-k) u\|_{H^{s}\left(\mathbf{R}^{n}\right)} .
$$

Now, if $v$ is a function on $M=(0,1) \times \mathbf{R}^{d}$ we define the even (resp. odd) periodic extensions on $\mathbf{T} \times \mathbf{R}^{d}, v^{\mathrm{ev}}$ (resp. $v^{\text {od }}$ ), by

$$
\begin{aligned}
& v^{\mathrm{ev}}\left(x_{1}, x^{\prime}\right)= \begin{cases}v\left(-x_{1}, x^{\prime}\right), & \text { if }-1<x_{1}<0, \\
v\left(x_{1}, x^{\prime}\right), & \text { if } 0 \leq x_{1}<1, \\
v\left(x_{1}-2 k, x^{\prime}\right), & \text { if } x_{1}-2 k \in(-1,1), k \in \mathbf{Z} .\end{cases} \\
& v^{\mathrm{od}}\left(x_{1}, x^{\prime}\right)= \begin{cases}-v\left(-x_{1}, x^{\prime}\right), & \text { if }-1<x_{1}<0, \\
v\left(x_{1}, x^{\prime}\right), & \text { if } 0 \leq x_{1}<1, \\
v\left(x_{1}-2 k, x^{\prime}\right), & \text { if } x_{1}-2 k \in(-1,1), k \in \mathbf{Z} .\end{cases}
\end{aligned}
$$


Corollary 6.7. Let $M=(0,1) \times \mathbf{R}$.

(1) Assume $0 \leq s<\frac{3}{2}$; then the map $v \mapsto v^{\mathrm{ev}}$ is continuous from $H_{u l}^{s}(M)$ to $H_{u l}^{s}\left(\mathbf{R}^{2}\right)$.

(2) Assume $\frac{3}{2}<s<\frac{7}{2}$. Let

$$
E_{s}=\left\{u \in H_{u l}^{s}(M): \partial_{x_{1}} u\left(\varepsilon, x_{2}\right)=0, \varepsilon=0,1, \forall x_{2} \in \mathbf{R}\right\} .
$$

Then the map $v \mapsto v^{\mathrm{ev}}$ is continuous from $E_{s}$ to $H_{u l}^{s}\left(\mathbf{R}^{2}\right)$.

(3) Assume $0 \leq s<\frac{1}{2}$; then the map $v \mapsto v^{\text {od }}$ is continuous from $H_{u l}^{s}(M)$ to $H_{u l}^{s}\left(\mathbf{R}^{2}\right)$.

(4) Assume $\frac{1}{2}<s<\frac{5}{2}$. Let

$$
F_{s}=\left\{u \in H_{u l}^{s}(M): u\left(\varepsilon, x_{2}\right)=0, \varepsilon=0,1, \forall x_{2} \in \mathbf{R}\right\} .
$$

Then the map $v \mapsto v^{\text {od }}$ is continuous from $F_{s}$ to $H_{u l}^{s}\left(\mathbf{R}^{2}\right)$.

(5) Assume $\frac{5}{2}<s \leq 4$. Let

$$
G_{s}=\left\{u \in H_{u l}^{s}(M): u\left(\varepsilon, x_{2}\right)=\partial_{x_{1}}^{2} u\left(\varepsilon, x_{2}\right)=0, \varepsilon=0,1, \forall x_{2} \in \mathbf{R}\right\} .
$$

Then the map $v \mapsto v^{\text {od }}$ is continuous from $G_{s}$ to $H_{u l}^{s}\left(\mathbf{R}^{2}\right)$.

Proof. Since $\left(D_{x_{2}}^{\alpha} v\right)^{\mathrm{ev}}=D_{x_{2}}^{\alpha}\left(v^{\mathrm{ev}}\right),\left(D_{x_{2}}^{\alpha} v\right)^{\mathrm{od}}=D_{x_{2}}^{\alpha}\left(v^{\mathrm{od}}\right)$ the result is clearly a one dimensional result and it is enough to prove it for the one dimensional case, in which case it is a direct consequence of Proposition 6.6 and a localization argument.

Consider now an initial data $\left(\eta_{0}, \psi_{0}=\left.\phi_{0}\right|_{\Sigma_{0}}, V_{0}, B_{0}\right)$ satisfying the assumptions in Theorem 6.3 and define

$$
\tilde{\eta}_{0}=\eta_{0}^{\mathrm{ev}}, \quad \tilde{\psi}_{0}=\psi_{0}^{\mathrm{ev}}, \quad \widetilde{V}_{0, x_{1}}=V_{0, x_{1}}^{\mathrm{od}}, \quad \widetilde{V}_{0, x_{2}}=V_{0, x_{2}}^{\mathrm{ev}}, \quad \widetilde{B}_{0}=B_{0}^{\mathrm{ev}}
$$

on $\mathbf{T} \times \mathbf{R}$.

Recall (see Theorem 6.3) that, with $M=(0,1) \times \mathbf{R}$, we have set

$$
\mathcal{H}^{s}(M)=H_{u l}^{s+\frac{1}{2}}(M) \times L_{u l}^{2}(M) \times H_{u l}^{s}(M) \times H_{u l}^{s}(M),
$$

and introduce

$$
\mathcal{H}^{s}\left(\mathbf{R}^{2}\right)=H_{u l}^{s+\frac{1}{2}}\left(\mathbf{R}^{2}\right) \times L_{u l}^{2}\left(\mathbf{R}^{2}\right) \times H_{u l}^{s}\left(\mathbf{R}^{2}\right) \times H_{u l}^{s}\left(\mathbf{R}^{2}\right) .
$$

Then we have the following lemma.

Lemma 6.8. Let $2<s<3, s \neq \frac{5}{2}$ and $\left(\eta_{0}, \psi_{0}, V_{0}, B_{0}\right) \in \mathcal{H}^{s}(M)$ satisfying the hypothesis $\left(H_{1}\right)$ in Theorem 6.3. Then $\left(\tilde{\eta}_{0}, \tilde{\psi}_{0}, \widetilde{V}_{0}, \widetilde{B}_{0}\right) \in \mathcal{H}^{s}\left(\mathbf{R}^{2}\right)$ and are 2-periodic with respect to the $x_{1}$ variable.

Proof. This follows immediately from the hypothesis $\left(H_{1}\right)$ and Corollary 6.7 .

In the case of a rectangular basin, performing both reflection and periodizations with respect to the $x_{1}$ and the $x_{2}$ variables leads similarly to extensions

$$
\left(\tilde{\eta}_{0}, \tilde{\psi}_{0}, \widetilde{V}_{0}, \widetilde{B}_{0}\right) \in H_{u l}^{s+\frac{1}{2}}\left(\mathbf{R}^{2}\right) \times L_{u l}^{2}\left(\mathbf{R}^{2}\right) \times H_{u l}^{s}\left(\mathbf{R}^{2}\right) \times H_{u l}^{s}\left(\mathbf{R}^{2}\right)
$$

which are 2-periodic with respect to the $x_{1}$ variable and $2 L$ periodic with respect to the $x_{2}$ variable.

\subsubsection{Conclusion}

We are now in position to apply Theorem 2.3. We consider first the case of the canal. Starting from $\left(\eta_{0}, \psi_{0}, V_{0}, B_{0}\right)$, we define $\left(\tilde{\eta}_{0}, \tilde{\psi}_{0}, \widetilde{V}_{0}, \widetilde{B}_{0}\right)$ their periodized extensions following the process in Section 6.3.1. Let $(\tilde{\eta}, \tilde{v})$ be the solution of the free surface water waves system given by Theorem 2.3. Since the initial data $\left(\tilde{\eta}_{0}, \tilde{\psi}_{0}, \widetilde{V}_{0}^{\prime}, \widetilde{B}_{0}\right)$ are even while $\widetilde{V}_{0, x_{1}}$ is odd, our uniqueness result guarantees that the solution satisfies the same symmetry property (because if we consider our solution, the function obtained by symmetrization is also a solution with same initial data). The same 
argument shows that as the initial data are 2-periodic with respect to the variable $x_{1}$, so is the solution. As a consequence if we define $v, \eta, P$ as the trace of $\tilde{v}, \tilde{\eta}, \widetilde{P}$ on $(0,1) \times \mathbf{R}$, we get that they satisfy trivially the incompressible Euler equation with free surface

$$
\begin{aligned}
& \partial_{t} v+v \cdot \nabla_{x, y} v+\nabla_{x, y} P=-g e_{y}, \quad \operatorname{div}_{x, y} v=0, \quad \operatorname{curl}_{x, y} v=0 \quad \text { in } \Omega, \\
& \partial_{t} \eta=\sqrt{1+|\nabla \eta|^{2}} v \cdot v \quad \text { on } \Sigma, \\
& P=0 \quad \text { on } \Sigma,
\end{aligned}
$$

and to conclude on the existence point in Theorem 6.3, it only remains to check that the "solid wall condition"

$$
v \cdot n=0, \quad \text { on } \Gamma=\Gamma_{1} \cup \Gamma_{2}
$$

is satisfied. On $\Gamma_{1}$ it is a straightforward consequence of the condition $\tilde{v} \cdot \tilde{n}=0$, while on $\Gamma_{2}$ it is simply consequence of the fact that the component of the velocity field along $x_{1}, \tilde{v}_{x_{1}}$ is odd and 2-periodic. To prove the uniqueness part in Theorem 6.3, starting from a solution of (6.12), (6.13), on the time interval $[-T, T]$, if we define the function $\tilde{v}, \tilde{\eta}$ at each time $t$ following the same procedure, we end up with a solution of (6.2), (6.3) in the domain $\{(t, x, y) ; t \in$ $(-T, T),(x, y) \in \widetilde{\Omega}(t)\}$, at the same level of regularity. Indeed, the jump formula gives

$$
\partial_{t} \tilde{v}+\tilde{v} \cdot \nabla_{x, y} \tilde{v}+\nabla_{x, y} \tilde{P}=-g e_{y}+\left[v_{x_{1}} \cdot \partial_{x_{1}} v\right] \otimes \delta_{\Gamma_{2}}=-g e_{y},
$$

where in the last equality we used that the component of the velocity field along $x_{1}$ vanishes on $\Gamma_{2}$. The uniqueness part in Theorem 6.3 consequently follows from the uniqueness part in Theorem 2.3. The case of a rectangular basin is similar.

\section{Technical results}

\subsection{Invariance}

The following result shows that the definition of the uniformly local Sobolev spaces does not depend on the choice of the function $\chi$ satisfying (2.3).

Lemma 7.1. Let $E$ be a normed space of functions from $\mathbf{R}^{d}$ to $\mathbf{C}$ such that

$$
\forall \theta \in W^{\infty, \infty}\left(\mathbf{R}^{d}\right) \quad \exists C>0: \quad\|\theta u\|_{E} \leq C\|u\|_{E} \quad \forall u \in E
$$

where $C$ depends only on a finite number of semi-norms of $\theta$ in $W^{\infty, \infty}\left(\mathbf{R}^{d}\right)$. Let $\chi \in C_{0}^{\infty}\left(\mathbf{R}^{d}\right)$ satisfying (2.3). Then for any $\tilde{\chi} \in C_{0}^{\infty}\left(\mathbf{R}^{d}\right)$ there exists $C^{\prime}>0$ such that

$$
\sup _{k \in \mathbf{Z}^{d}}\left\|\tilde{\chi}_{k} u\right\|_{E} \leq C^{\prime} \sup _{q \in \mathbf{Z}^{d}}\left\|\chi_{q} u\right\|_{E}
$$

where $\chi_{q}(x)=\chi(x-q)$ and $\tilde{\chi}_{k}(x)=\tilde{\chi}(x-k)$.

Proof. Let $\underline{\chi} \in C_{0}^{\infty}\left(\mathbf{R}^{d}\right)$ be equal to one on the support of $\chi$. We write with $N=d+1$

$$
\tilde{\chi}_{k} \chi_{q} u=\langle k-q\rangle^{-N}\left[\frac{\langle k-q\rangle^{N}}{\langle x-q\rangle^{N}} \tilde{\chi}_{k}\right]\left[\langle x-q\rangle^{N} \underline{\chi}_{q}\right] \chi_{q} u .
$$

Since the two functions inside the brackets belong to $W^{\infty, \infty}\left(\mathbf{R}^{d}\right)$ with semi-norms independent of $k, q$, using the assumption in the lemma we deduce that

$$
\left\|\tilde{\chi}_{k} u\right\|_{E} \leq \sum_{q \in \mathbf{Z}^{d}}\left\|\tilde{\chi}_{k} \chi_{q} u\right\|_{E} \leq C \sum_{q \in \mathbf{Z}^{d}}\langle k-q\rangle^{-N} \sup _{q \in \mathbf{Z}^{d}}\left\|\chi_{q} u\right\|_{E},
$$

which completes the proof. 
Lemma 7.2. Let $\mu \in \mathbf{R}$ and $N \geq d+1$. Then there exists $C>0$ such that

$$
\sup _{x \in \mathbf{R}^{d}}\left\|\langle x-\cdot\rangle^{-N} u\right\|_{H^{\mu}\left(\mathbf{R}^{d}\right)} \leq C\|u\|_{H_{u l}^{\mu}\left(\mathbf{R}^{d}\right)}
$$

for all $u \in H_{u l}^{\mu}\left(\mathbf{R}^{d}\right)$.

Proof. Indeed we have

$$
\left\|\langle x-\cdot\rangle^{-N} u\right\|_{H^{\mu}} \leq \sum_{q \in \mathbf{Z}^{d}}\left\|\langle x-\cdot\rangle^{-N} \chi_{q} u\right\|_{H^{\mu}}
$$

and we write

$$
\langle x-y\rangle^{-N} \chi_{q}(y) u(y)=\frac{1}{\langle x-q\rangle^{N}} \frac{\langle x-q\rangle^{N}}{\langle x-y\rangle^{N}} \tilde{\chi}_{q}(y) \chi_{q}(y) u(y)
$$

where $\tilde{\chi} \in C_{0}^{\infty}\left(\mathbf{R}^{d}\right), \tilde{\chi}=1$ on the support of $\chi$. This implies that

$$
\sum_{q \in \mathbf{Z}^{d}}\left\|\langle x-\cdot\rangle^{-N} \chi_{q} u\right\|_{H^{\mu}} \leq C_{N} \sum_{q \in \mathbf{Z}^{d}} \frac{1}{\langle x-q\rangle^{N}}\|u\|_{H_{u l}^{\mu}} \leq C_{N}^{\prime}\|u\|_{H_{u l}^{\mu}},
$$

since the function $y \mapsto \frac{\langle x-q\rangle^{N}}{\langle x-y\rangle^{N}} \widetilde{\chi}_{q}(y)$ belongs to $W^{\infty, \infty}\left(\mathbf{R}^{d}\right)$ with semi-norms uniformly bounded (independently of $x$ and $q$ ).

\subsection{Product laws}

Proposition 7.3. (i) Let $\sigma_{j} \in \mathbf{R}, j=1,2$ be such that $\sigma_{1}+\sigma_{2}>0$ and $u_{j} \in H_{u l}^{\sigma_{j}}\left(\mathbf{R}^{d}\right), j=1,2$. Then $u_{1} u_{2} \in H_{u l}^{\sigma_{0}}\left(\mathbf{R}^{d}\right)$ for $\sigma_{0} \leq \sigma_{j}$ and $\sigma_{0}<\sigma_{1}+\sigma_{2}-\frac{d}{2}$. Moreover we have

$$
\left\|u_{1} u_{2}\right\|_{H_{u l}^{\sigma_{0}}\left(\mathbf{R}^{d}\right)} \leq C\left\|u_{1}\right\|_{H_{u l}^{\sigma_{1}}\left(\mathbf{R}^{d}\right)}\left\|u_{2}\right\|_{H_{u l}^{\sigma_{2}}\left(\mathbf{R}^{d}\right)} .
$$

(ii) Let $s \geq 0$ and $u_{j} \in H_{u l}^{s}\left(\mathbf{R}^{d}\right) \cap L^{\infty}\left(\mathbf{R}^{d}\right), j=1,2$. Then $u_{1} u_{2} \in H_{u l}^{s}\left(\mathbf{R}^{d}\right)$ and

$$
\left\|u_{1} u_{2}\right\|_{H_{u l}^{s}\left(\mathbf{R}^{d}\right)} \leq C\left(\left\|u_{1}\right\|_{L^{\infty}\left(\mathbf{R}^{d}\right)}\left\|u_{2}\right\|_{H_{u l}^{s}\left(\mathbf{R}^{d}\right)}+\left\|u_{2}\right\|_{L^{\infty}\left(\mathbf{R}^{d}\right)}\left\|u_{1}\right\|_{H_{u l}^{s}\left(\mathbf{R}^{d}\right)}\right) .
$$

(iii) Let $F \in C^{\infty}\left(\mathbf{R}^{N}, \mathbf{C}\right)$ be such that $F(0)=0$. Let $s>\frac{d}{2}$. If $U \in\left(H_{u l}^{s}\left(\mathbf{R}^{d}\right)\right)^{N}$ then $F(U) \in H_{u l}^{s}\left(\mathbf{R}^{d}\right)$ and

$$
\|F(U)\|_{H_{u l}^{s}\left(\mathbf{R}^{d}\right)} \leq G\left(\|U\|_{\left(L^{\infty}\left(\mathbf{R}^{d}\right)\right)^{N}}\right)\|U\|_{\left(H_{u l}^{s}\left(\mathbf{R}^{d}\right)\right)^{N}}
$$

for an increasing function $G: \mathbf{R}^{+} \rightarrow \mathbf{R}^{+}$.

Proof. The proofs are straightforward extensions of the proofs in the classical Sobolev spaces case. Indeed let us show $(i)$ for instance. Let $\chi_{q}$ be defined in (2.3) and $\tilde{\chi} \in C_{0}^{\infty}\left(\mathbf{R}^{d}\right)$ be equal to one on the support of $\chi$. Then from the classical case we can write

$$
\begin{aligned}
\left\|\chi_{q} u_{1} u_{2}\right\|_{H^{\sigma_{0}}} & =\left\|\chi_{q} u_{1} \tilde{\chi}_{q} u_{2}\right\|_{H^{\sigma_{0}}} \leq C\left\|\chi_{q} u_{1}\right\|_{H^{\sigma_{1}}}\left\|\tilde{\chi}_{q} u_{2}\right\|_{H^{\sigma_{2}}} \\
& \leq C\left\|u_{1}\right\|_{H_{u l}^{\sigma_{1}}\left\|u_{2}\right\|_{H_{u l}^{\sigma_{2}}} .}
\end{aligned}
$$

The proofs of (ii) and (iii) are similar. 
The following spaces will be used in the sequel:

Definition 7.4. Let $p \in[1,+\infty], J=\left(z_{0}, 0\right), z_{0}<0$ and $\sigma \in \mathbf{R}$.

(1) The space $L^{p}\left(J, H^{\sigma}\left(\mathbf{R}^{d}\right)\right)_{u l}$ is defined as the space of measurable functions $u$ from $\mathbf{R}_{x}^{d} \times J_{z}$ to $\mathbf{C}$ such that

$$
\|u\|_{L^{p}\left(J, H^{\sigma}\left(\mathbf{R}^{d}\right)\right)_{u l}}:=\sup _{q \in \mathbf{Z}^{d}}\left\|\chi_{q} u\right\|_{L^{p}\left(J, H^{\sigma}\left(\mathbf{R}^{d}\right)\right)}<+\infty .
$$

(2) We set

$$
\begin{aligned}
& X_{u l}^{\sigma}(J)=L^{\infty}\left(J, H^{\sigma}\left(\mathbf{R}^{d}\right)\right)_{u l} \cap L^{2}\left(J, H^{\sigma+\frac{1}{2}}\left(\mathbf{R}^{d}\right)\right)_{u l} \\
& Y_{u l}^{\sigma}(J)=L^{1}\left(J, H^{\sigma}\left(\mathbf{R}^{d}\right)\right)_{u l}+L^{2}\left(J, H^{\sigma-\frac{1}{2}}\left(\mathbf{R}^{d}\right)\right)_{u l}
\end{aligned}
$$

endowed with their natural norms.

(3) We define the spaces $X^{\sigma}(J), Y^{\sigma}(J)$ by the same formulas without the subscript $u l$.

Notice that $L^{\infty}\left(J, H^{\sigma}\left(\mathbf{R}^{d}\right)\right)_{u l}=L^{\infty}\left(J, H_{u l}^{\sigma}\left(\mathbf{R}^{d}\right)\right)$.

Lemma 7.5. Let $\sigma_{0}, \sigma_{1}, \sigma_{2}$ be real numbers such that $\sigma_{1}+\sigma_{2}>0, \sigma_{0} \leq \sigma_{j}, j=1,2, \sigma_{0}<\sigma_{1}+\sigma_{2}-\frac{d}{2}$ and $1 \leq$ $p \leq+\infty$. Then

$$
\|u v\|_{L^{p}\left(J, H^{\sigma_{0}}\left(\mathbf{R}^{d}\right)\right)_{u l}} \leq C\|u\|_{L^{\infty}\left(J, H^{\sigma_{1}}\left(\mathbf{R}^{d}\right)\right)_{u l}}\|v\|_{L^{p}\left(J, H^{\sigma_{2}}\left(\mathbf{R}^{d}\right)\right)_{u l}}
$$

whenever the right hand side is finite.

The same inequality holds for the spaces without the subscript ul.

Proof. This follows immediately from Proposition 7.3(i) and (7.1).

Lemma 7.6. If $\sigma>\frac{d}{2}$ the spaces $X_{u l}^{\sigma}(J)$ and $X^{\sigma}(J)$ are algebras.

Lemma 7.7. Let $s_{0}>1+\frac{d}{2}, \mu>0$ and $J=(-1,0)$. Then we have

$$
\begin{aligned}
\|f g\|_{X_{u l}^{\mu}} & \leq C\left(\|f\|_{L^{\infty}\left(J, H^{s_{0}-1}\right)_{u l}}\|g\|_{X_{u l}^{\mu}}+\|g\|_{L^{\infty}\left(J, H^{s_{0}-1}\right)_{u l}}\|f\|_{X_{u l}^{\mu}}\right), \\
\|f g\|_{X_{u l}^{\mu}} & \leq C\left(\|f\|_{L^{\infty}\left(J, H^{s_{0}-1}\right)_{u l}}\|g\|_{X_{u l}^{\mu}}+\|g\|_{L^{\infty}\left(J, H^{s_{0}-\frac{3}{2}}\right)_{u l}}\|f\|_{\left.X_{u l}^{\mu+\frac{1}{2}}\right) .}\right.
\end{aligned}
$$

Let $F \in C^{\infty}\left(\mathbf{R}^{N}, \mathbf{C}\right)$ be such that $F(0)=0$. Then there exists a non-decreasing function $\mathcal{F}: \mathbf{R}^{+} \rightarrow \mathbf{R}^{+}$such that for $\mu>\frac{d}{2}$ we have

$$
\|F(U)\|_{X_{u l}^{\mu}} \leq \mathcal{F}\left(\|U\|_{L^{\infty}\left(J, H^{s_{0}-1}\right) u l}\right)\|U\|_{X_{u l}^{\mu}}
$$

Proof. The first and the third estimates follow easily from (ii), (iii) in Proposition 7.3. To prove the second one we start from the inequality (see [2, Corollary 2.12])

$$
\left\|\chi_{k} f g\right\|_{H^{t}} \leq C\left(\left\|\chi_{k} f\right\|_{L^{\infty}}\left\|\tilde{\chi}_{k} g\right\|_{H^{t}}+\left\|\tilde{\chi}_{k} g\right\|_{C_{*}^{-\frac{1}{2}}}\left\|\chi_{k} f\right\|_{H^{t+\frac{1}{2}}}\right), \quad t>0
$$

where $\tilde{\chi} \in C_{0}^{\infty}\left(\mathbf{R}^{d}\right)$ is equal to one on the support of $\chi$. Then we use the continuous embeddings: $H^{s_{0}-1} \subset L^{\infty}$, $H^{s_{0}-\frac{3}{2}} \subset C_{*}^{-\frac{1}{2}}$ and the above inequality for $t=\mu, t=\mu+\frac{1}{2}$. 


\subsection{Continuity of the pseudo-differential operators}

We have the following result which reflects the pseudo-local character of the pseudo-differential operators. Recall that $S_{1,0}^{m}$ is the set of symbols $p \in C^{\infty}\left(\mathbf{R}^{d} \times \mathbf{R}^{d}\right)$ such that

$$
\left|D_{\xi}^{\alpha} D_{x}^{\beta} p(x, \xi)\right| \leq C_{\alpha, \beta}(1+|\xi|)^{m-|\alpha|} \quad \forall \alpha, \beta \in \mathbf{N}^{d}, \forall(x, \xi) \in \mathbf{R}^{d} \times \mathbf{R}^{d} .
$$

Proposition 7.8. Let $P$ be a pseudo-differential operator whose symbol belongs to the class $S_{1,0}^{m}$. Then for every $s \in \mathbf{R}$ there exists a constant $C>0$ such that

$$
\|P u\|_{H_{u l}^{s}\left(\mathbf{R}^{d}\right)} \leq C\|u\|_{H_{u l}^{s+m}\left(\mathbf{R}^{d}\right)},
$$

for every $u \in H_{u l}^{s+m}\left(\mathbf{R}^{d}\right)$, where $C$ depends only on semi-norms of the symbol in $S_{1,0}^{m}$.

Proof. Write

$$
\chi_{k} P u=\sum_{|k-q| \leq 2} \chi_{k} P \chi_{q} u+\sum_{|k-q| \geq 3} \chi_{k} P \chi_{q} u=: A+\sum_{|k-q| \geq 3} B_{k, q} .
$$

The first sum is finite depending only on the dimension. To bound it in $H^{s}\left(\mathbf{R}^{d}\right)$ we use the usual continuity of pseudo-differential operators. For the second one let $n_{0} \in \mathbf{N}, n_{0} \geq s$. We shall prove that

$$
\left\|D_{x}^{\alpha} B_{k, q}\right\|_{L^{2}\left(\mathbf{R}^{d}\right)} \leq \frac{C_{d}}{\langle k-q\rangle^{d+1}}\|u\|_{H_{u l}^{s+m}}, \quad|\alpha| \leq n_{0}
$$

which will complete the proof of Proposition 7.8.

Notice that, due to the presence of $\chi_{k}$, we have $\left\|D_{x}^{\alpha} B_{k, q}\right\|_{L^{2}} \leq C\left\|D_{x}^{\alpha} B_{k, q}\right\|_{L^{\infty}}$. We have

$$
D_{x}^{\alpha} B_{k, q}(x)=\left\langle D_{x}^{\alpha} K(x, \cdot), \chi_{q} u\right\rangle
$$

with

$$
K(x, y)=(2 \pi)^{-d} \int_{\mathbf{R}^{d}} e^{i(x-y) \cdot \xi} p(x, \xi) d \xi \chi_{k}(x) \tilde{\chi}_{q}(y)
$$

where $\tilde{\chi} \in C_{0}^{\infty}\left(\mathbf{R}^{d}\right), \tilde{\chi}=1$ on the support of $\chi$.

Now on the support of $\chi_{k}(x) \tilde{\chi}_{q}(y)$ we have $|x-y| \geq \delta|k-q|, \delta>0$. Integrating by parts $N$ times (with large $N$ depending on $d, n_{0}$ ) with the vector field $L=\sum_{j=1}^{d} \frac{x_{j}-y_{j}}{|x-y|^{2}} \partial_{\xi_{j}}$ we see that for all $\beta \in \mathbf{N}^{d}$ we have

$$
\left|D_{x}^{\beta} K(x, y)\right| \leq \frac{C_{d, \beta}}{\langle k-q\rangle^{d+1}}\left|\tilde{\chi}_{q}(y)\right|, \quad \forall(x, y) \in \mathbf{R}^{d} \times \mathbf{R}^{d} .
$$

It follows that

$$
\begin{aligned}
\left|D_{x}^{\alpha} B_{k, q}(x)\right| & \leq\left\|D_{x}^{\alpha} K(x, \cdot)\right\|_{H^{-(s+m)}}\left\|\chi_{q} u\right\|_{H^{s+m}} \\
& \leq \frac{C_{d, \beta}}{\langle k-q\rangle^{d+1}}\left\|\chi_{q} u\right\|_{H^{s+m}}
\end{aligned}
$$

which proves (7.7) and hence concludes the proof.

In a particular case the proof above gives the following more precise result.

Proposition 7.9. Let $m \in \mathbf{R}, h(\xi)=\tilde{h}\left(\frac{\xi}{|\xi|}\right)|\xi|^{m} \psi(\xi)$ where $\tilde{h} \in C^{\infty}\left(\mathbf{S}^{d-1}\right)$ and $\psi \in C^{\infty}\left(\mathbf{R}^{d}\right)$ is such that $\psi(\xi)=1$ if $|\xi| \geq 1, \psi(\xi)=0$ if $|\xi| \leq \frac{1}{2}$. Then for every $\mu \in \mathbf{R}$ there exists a constant $C$ such that

$$
\left\|h\left(D_{x}\right) u\right\|_{H_{u l}^{\mu}\left(\mathbf{R}^{d}\right)} \leq C\|\tilde{h}\|_{H^{d+1}\left(\mathbf{S}^{d-1}\right)}\|u\|_{H_{u l}^{\mu+m}\left(\mathbf{R}^{d}\right)}
$$

for all $u \in H_{u l}^{\mu+m}\left(\mathbf{R}^{d}\right)$. 
We shall use the following result when $p(\xi)=\langle\xi\rangle$ and $p(\xi)=|\xi|^{2}$.

Lemma 7.10. Let $d \geq 1, r>0, m \in \mathbf{R}$. Let $p \in S_{1,0}^{r}\left(\mathbf{R}^{d}\right), a \in S_{1,0}^{m}\left(\mathbf{R}^{d}\right)$ be two symbols with constant coefficients. We assume that one can find $c_{0}>0$ such that for all $\xi \in \mathbf{R}^{d}$ we have $p(\xi) \geq c_{0}|\xi|^{r}$. Then for all $\sigma \in \mathbf{R}$ and every interval $I=[0, T]$, one can find a positive constant $C$ such that, with $H^{s}=H^{s}\left(\mathbf{R}^{d}\right)$

$$
\left\|e^{-t p\left(D_{x}\right)} a\left(D_{x}\right) u\right\|_{L^{\infty}\left(I, H^{\sigma}\right)_{u l}}+\left\|e^{-t p\left(D_{x}\right)} a\left(D_{x}\right) u\right\|_{L^{2}\left(I, H^{\sigma+\frac{r}{2}}\right)_{u l}} \leq C\|u\|_{H_{u l}^{\sigma+m}}
$$

for all $u \in H_{u l}^{\sigma+m}$.

Proof. The estimate of the first term in (7.8) follows from Proposition 7.8 since $e^{-t p(D)} a(D)$ is a pseudo-differential operator of order $m$ whose symbol has semi-norms in $S_{1,0}^{m}$ bounded by constants depending only on $T$. Let us look at the second term. Set

$$
I_{q}=\left\|\chi_{q} e^{-t p(D)} a(D) u\right\|_{L^{2}\left(I, H^{\sigma+\frac{r}{2}}\right)} .
$$

One can write

$$
\left\{\begin{array}{l}
I_{q}=A_{q}+B_{q} \\
A_{q}=\sum_{|k-q| \leq 2}\left\|\chi_{q} e^{-t p\left(D_{x}\right)} a\left(D_{x}\right) \chi_{k} u\right\|_{L^{2}\left(I, H^{\sigma+\frac{r}{2}}\right)}, \\
B_{q}=\sum_{|k-q| \geq 3}\left\|\chi_{q} e^{-t p\left(D_{x}\right)} a\left(D_{x}\right) \chi_{k} u\right\|_{L^{2}\left(I, H^{\sigma+\frac{r}{2}}\right)} .
\end{array}\right.
$$

Since the number of terms in the sum defining $A_{q}$ is bounded by a fixed constant (depending only on $d$ ) using a classical computation we can write

$$
\begin{aligned}
& A_{q} \leq C_{1} \sup _{k \in \mathbf{Z}^{d}}\left\|e^{-t p\left(D_{x}\right)} a\left(D_{x}\right) \chi_{k} u\right\|_{L^{2}\left(I, H^{\sigma+\frac{r}{2}}\right)} \\
& A_{q} \leq C_{2} \sup _{k \in \mathbf{Z}^{d}}\left\|a\left(D_{x}\right) \chi_{k} u\right\|_{H^{\sigma}} \leq C_{3} \sup _{k \in \mathbf{Z}^{d}}\left\|\chi_{k} u\right\|_{H^{\sigma+m}} \leq C_{3}\|u\|_{H_{u l}^{\sigma+m}} .
\end{aligned}
$$

Let us look at the term $B_{q}$. Let $N_{0}$ be an integer such that $N_{0} \geq \sigma+\frac{r}{2}$. Then $B_{q}$ is bounded by a finite sum of terms of the form

$$
\sum_{|k-q| \geq 3}\left\|\left(D_{x}^{\alpha} \chi_{q}\right)\left(D_{x}^{\beta} e^{-t p\left(D_{x}\right)} a\left(D_{x}\right)\right) \chi_{k} u\right\|_{L^{2}\left(I, L^{2}\right)}
$$

with $|\alpha|+|\beta| \leq N_{0}$. Due to the presence of the function $D^{\alpha} \chi_{q}, B_{q}$ is therefore bounded by a finite number of terms of the form

$$
\sum_{|k-q| \geq 3}\left\|\left(D_{x}^{\alpha} \chi_{q}\right)\left(D_{x}^{\beta} e^{-t p\left(D_{x}\right)} a\left(D_{x}\right)\right) \chi_{k} u\right\|_{L^{2}\left(I, L^{\infty}\right) .}
$$

Now we can write

$$
F(t, x):=\left(D_{x}^{\alpha} \chi_{q}\right)\left(D_{x}^{\beta} e^{-t p\left(D_{x}\right)} a\left(D_{x}\right)\right) \chi_{k} u(x)=\left\langle K(t, x, \cdot),\left(\chi_{k} u\right)(\cdot)\right\rangle
$$

with

$$
K(t, x, y)=(2 \pi)^{-d}\left(D_{x}^{\alpha} \chi_{q}\right)(x) \tilde{\chi}_{k}(y) \int_{\mathbf{R}^{d}} e^{i(x-y) \cdot \xi} q(t, \xi) d \xi
$$

where $\tilde{\chi} \in C_{0}^{\infty}\left(\mathbf{R}^{d}\right)$ is equal to one on the support of $\chi$ and $q(t, \xi)=\xi^{\beta} e^{-t p(\xi)} a(\xi)$. It follows that for fixed $(t, x)$ we have

$$
|F(t, x)| \leq\|K(t, x, \cdot)\|_{H^{-(\sigma+m)}}\left\|\chi_{k} u\right\|_{H^{\sigma+m}} .
$$


Let $N_{1} \in \mathbf{N}$ be fixed such that $N_{1} \geq-(\sigma+m)$. We shall show that for every $N \in \mathbf{N}$ one can find $C_{N}=C_{N}(T)>0$ such that for every $(t, x) \in I \times \mathbf{R}^{d}$ we have

$$
\|K(t, x, \cdot)\|_{H^{N_{1}}} \leq \frac{C_{N}}{\langle k-q\rangle^{N}}\left|\left(D^{\alpha} \chi_{q}\right)(x)\right| .
$$

Indeed for $|\mu| \leq N_{1}, D_{y}^{\mu} K(t, x, y)$ is a finite linear combination of terms of the form

$$
J(t, x, y):=\left(D_{x}^{\alpha} \chi_{q}\right)(x)\left(D_{y}^{\nu} \tilde{\chi}_{k}\right)(y) \int e^{i(x-y) \cdot \xi} \xi^{\lambda} q(t, \xi) d \xi
$$

where $|\nu|+|\lambda|=|\mu|$.

We notice that for all $\gamma \in \mathbf{N}^{d}$ we have

$$
\left|D_{\xi}^{\gamma}\left(\xi^{\lambda} q(t, \xi)\right)\right| \leq C_{\gamma}(T)\langle\xi\rangle^{N_{0}+N_{1}+m-|\gamma|} .
$$

Now let $N \in \mathbf{N}$ be such that $N \geq \max \left(d+1, N_{0}+N_{1}+m+d+1\right)$ and $\gamma \in \mathbf{N}^{d}$ with $|\gamma|=N$. Then

$$
(x-y)^{\gamma} J(t, x, y)=\left(D_{x}^{\alpha} \chi_{q}\right)(x)\left(D_{y}^{v} \tilde{\chi}_{k}\right)(y) \int e^{i(x-y) \cdot \xi}\left(-D_{\xi}\right)^{\gamma}\left(\xi^{\lambda} q(t, \xi)\right) d \xi .
$$

It follows from (7.14) that

$$
\left|(x-y)^{\gamma} J(t, x, y)\right| \leq C_{1}(T)\left|\left(D_{x}^{\alpha} \chi_{q}\right)(x)\right|\left|\left(D_{y}^{v} \tilde{\chi}_{k}\right)(y)\right| .
$$

Now since $|k-q| \geq 3$, on the support of $\left(D_{x}^{\alpha} \chi_{q}\right)(x)\left(D_{y}^{v} \tilde{\chi}_{k}\right)(y)$ we have $|x-y| \geq \frac{1}{3}|k-q|$. It follows that

$$
|J(t, x, y)| \leq \frac{C_{2}(T)}{\langle k-q\rangle^{N}}\left|\left(D_{x}^{\alpha} \chi_{q}\right)(x)\right|\left|\left(D_{y}^{v} \tilde{\chi}_{k}\right)(y)\right|
$$

which proves (7.13). According to (7.12) and (7.13) we obtain

$$
\|F\|_{L^{2}\left(I, L^{\infty}\right)} \leq \frac{C_{3}(T)}{\langle k-q\rangle^{N}}\left\|\chi_{k} u\right\|_{H^{\sigma+m}} \leq \frac{C_{3}(T)}{\langle k-q\rangle^{N}}\|u\|_{H_{u l}^{\sigma+m}}
$$

which implies that $B_{q} \leq C_{4}(T)\|u\|_{H_{u l}^{\sigma+m}}$. Combined with (7.9) and (7.10) this proves the estimate of the second term in (7.8).

Corollary 7.11. Let $m \in \mathbf{R}$ and $a \in S_{1,0}^{m}\left(\mathbf{R}^{d}\right)$. Then for every $\sigma \in \mathbf{R}$ there exists $C>0$ such that

$$
\left\|e^{\delta z\left\langle D_{x}\right\rangle} a\left(D_{x}\right) u\right\|_{X_{u l}^{\sigma-\frac{1}{2}}(-1,0)} \leq C\|u\|_{H_{u l}^{\sigma+m-\frac{1}{2}}}\left(\mathbf{R}^{d}\right)
$$

for every $\delta>0$ and every $u \in H_{u l}^{\sigma+m-\frac{1}{2}}\left(\mathbf{R}^{d}\right)$.

\subsection{An interpolation lemma}

We shall use the following interpolation lemma for which we refer to [27, Théorème 3.1].

Lemma 7.12. Let $J=(-1,0)$ and $t \in \mathbf{R}$. Let $f \in L_{z}^{2}\left(J, H^{t+\frac{1}{2}}\left(\mathbf{R}^{d}\right)\right)$ be such that $\partial_{z} f \in L_{z}^{2}\left(J, H^{t-\frac{1}{2}}\left(\mathbf{R}^{d}\right)\right)$. Then $f \in C_{z}^{0}\left([-1,0], H^{t}\left(\mathbf{R}^{d}\right)\right)$ and there exists an absolute constant $C>0$ such that

$$
\sup _{z \in[-1,0]}\|f(z, \cdot)\|_{H^{t}\left(\mathbf{R}^{d}\right)} \leq C\|f\|_{L_{z}^{2}\left(J, H^{\left.t+\frac{1}{2}\left(\mathbf{R}^{d}\right)\right)}\right.}+C\left\|\partial_{z} f\right\|_{L_{z}^{2}\left(J, H^{t-\frac{1}{2}}\left(\mathbf{R}^{d}\right)\right)} .
$$




\subsection{Paradifferential operators}

\subsubsection{Symbolic calculus}

In this section we quote some results which concern the symbolic calculus for paradifferential operators in the framework of the uniformly local Sobolev spaces. Of course, here, the theory for the classical Sobolev spaces will be assumed to be known (see [8] and [28]).

The following technical lemma will be used in the sequel. Here $D=D_{x}$.

Lemma 7.13. Let $\chi \in C_{0}^{\infty}\left(\mathbf{R}^{d}\right)$ and $\tilde{\chi} \in C_{0}^{\infty}\left(\mathbf{R}^{d}\right)$ be equal to one on the support of $\chi$. Let $\psi, \theta \in \mathcal{S}\left(\mathbf{R}^{d}\right)$. For every $m, \sigma \in \mathbf{R}$ one can find a constant $C>0$ such that

$$
\sum_{j \geq-1}\left\|\chi_{k} \psi\left(2^{-j} D\right)\left(\left(1-\tilde{\chi}_{k}\right) u\right) \theta\left(2^{-j} D\right) v\right\|_{H^{m}\left(\mathbf{R}^{d}\right)} \leq C\|u\|_{H_{u l}^{\sigma}\left(\mathbf{R}^{d}\right)}\|v\|_{L^{\infty}\left(\mathbf{R}^{d}\right)} .
$$

For every $m, \sigma, t \in \mathbf{R}$ one can find a constant $C>0$ such that

$$
\sum_{j \geq-1}\left\|\chi_{k} \psi\left(2^{-j} D\right)\left(\left(1-\tilde{\chi}_{k}\right) u\right) \theta\left(2^{-j} D\right) v\right\|_{H^{m}\left(\mathbf{R}^{d}\right)} \leq C\|u\|_{H_{u l}^{\sigma}\left(\mathbf{R}^{d}\right)}\|v\|_{H_{u l}^{t}\left(\mathbf{R}^{d}\right)}
$$

Proof. We may assume $m \in \mathbf{N}$. Let us call $A_{k, j}$ the term inside the sum in the left hand side of (7.15). Due to $\chi_{k}$, the term $A_{k, j}$ is bounded by finite sum of terms of the form

$$
A_{k, j, \alpha}:=2^{j m}\left\|\left(D^{\alpha_{1}} \chi_{k}\right) \psi_{\alpha_{2}}\left(2^{-j} D\right)\left(\left(1-\tilde{\chi}_{k}\right) u\right) \tilde{\chi}_{k} \theta_{\alpha_{3}}\left(2^{-j} D\right) v\right\|_{L^{\infty}}
$$

where $\left|\alpha_{1}\right|+\left|\alpha_{2}\right|+\left|\alpha_{3}\right| \leq m$ and $\psi_{\alpha_{2}}=x^{\alpha_{2}} \psi, \theta_{\alpha_{3}}=x^{\alpha_{3}} \theta$. We are going to show that for large $N \in \mathbf{N}$ we have

(i) $\quad\left\|\left(D^{\alpha_{1}} \chi_{k}\right) \psi_{\alpha_{2}}\left(2^{-j} D\right)\left(\left(1-\tilde{\chi}_{k}\right) u\right)\right\|_{L^{\infty}} \leq C_{N} 2^{j M_{1}(d, \sigma)} 2^{-j N}\|u\|_{H_{u l}^{\sigma}}$

(ii) $\left\|\tilde{\chi}_{k} \theta_{\alpha_{3}}\left(2^{-j} D\right) v\right\|_{L^{\infty}} \leq C\|v\|_{L^{\infty}}$

(iii) $\left\|\tilde{\chi}_{k} \theta_{\alpha_{3}}\left(2^{-j} D\right) v\right\|_{L^{\infty}} \leq C 2^{j M_{2}(d, t)}\|v\|_{H_{u l}^{t}}$

where, as indicated, $M_{j}$ are fixed constants depending only on $d, \sigma, t$. Then the lemma will follow from these estimates.

To prove $(i)$ we write

$$
\begin{aligned}
& \left(D^{\alpha_{1}} \chi_{k}(x)\right) \psi_{\alpha_{2}}\left(2^{-j} D\right)\left(\left(1-\tilde{\chi}_{k}\right) u\right)(x) \\
& \quad=(2 \pi)^{-d} 2^{j d} 2^{-j N} \times\left\langle\left(2^{j}|x-\cdot|\right)^{N} \widehat{\psi_{\alpha_{2}}}\left(2^{j}(\cdot-x)\right), \frac{\langle x-\cdot\rangle^{N}}{|x-|^{N}}\left(D^{\alpha_{1}} \chi_{k}(x)\right)\left(1-\tilde{\chi}_{k}(\cdot)\right)\langle x-\cdot\rangle^{-N} u\right\rangle .
\end{aligned}
$$

The function $y \mapsto \frac{\langle x-y\rangle^{N}}{|x-y|^{N}}\left(D^{\alpha_{1}} \chi_{k}(x)\right)\left(1-\tilde{\chi}_{k}(y)\right)$ belongs to $W^{\infty, \infty}$ with semi-norms uniformly bounded in $x$. Using the duality $H^{-\sigma}-H^{\sigma}$ we deduce that

$$
\left\|\left(D^{\alpha_{1}} \chi_{k}\right) \psi_{\alpha_{2}}\left(2^{-j} D\right)\left(\left(1-\tilde{\chi}_{k}\right) u\right)\right\|_{L^{\infty}} \leq C_{N} 2^{j M_{1}(d, \sigma)} 2^{-j N}\left\|\langle x-\cdot\rangle^{-N} u\right\|_{H^{\sigma}},
$$

and we conclude using Lemma 7.2.

The estimate (ii) is easy. To prove (iii) we take $\check{\chi} \in C_{0}^{\infty}\left(\mathbf{R}^{d}\right)$ equal to one on the support of $\tilde{\chi}$ and we write

$$
\left\|\tilde{\chi}_{k} \theta_{\alpha_{3}}\left(2^{-j} D\right) v\right\|_{L^{\infty}} \leq\left\|\tilde{\chi}_{k} \theta_{\alpha_{3}}\left(2^{-j} D\right) \check{\chi}_{k} v\right\|_{L^{\infty}}+\left\|\tilde{\chi}_{k} \theta_{\alpha_{3}}\left(2^{-j} D\right)\left(1-\check{\chi}_{k}\right) v\right\|_{L^{\infty}} .
$$

The second term is bounded exactly by the same method as $(i)$. For the first one we write

$$
\tilde{\chi}_{k} \theta_{\alpha_{3}}\left(2^{-j} D\right) \check{\chi}_{k} v(x)=(2 \pi)^{-d} 2^{j d} \tilde{\chi}_{k}(x)\left\langle\widehat{\theta_{\alpha_{3}}}\left(2^{j}(\cdot-x)\right), \check{\chi}_{k}(\cdot) v(\cdot)\right\rangle
$$

and we use the $H^{-t}-H^{t}$ duality. 
Remark 7.14. (i) Notice that the same estimates in (7.15), (7.16) hold if in the left hand side one $2^{-j}$ is replaced by $2^{-j-j_{0}}$ where $j_{0} \in \mathbf{Z}$ is fixed.

(ii) Notice also that in the above proof we have proved that for all real numbers $m, \sigma$, all $N \in \mathbf{N}$ and all $\psi \in C_{0}^{\infty}\left(\mathbf{R}^{d}\right)$ one can find a positive constant $C_{N, m, \sigma}$ such that

$$
\left\|\chi_{k} \psi\left(2^{-j} D\right)\left(1-\tilde{\chi}_{k}\right) u\right\|_{H^{m}\left(\mathbf{R}^{d}\right)} \leq C_{N, m, \sigma} 2^{-j N}\|u\|_{H_{u l}^{\sigma}\left(\mathbf{R}^{d}\right)}
$$

for every $j \in \mathbf{N}$ and every $k \in \mathbf{Z}^{d}$.

We introduce now the para-differential calculus.

Definition 7.15. Given $m \in \mathbf{R}, \rho \in[0,1], \Gamma_{\rho}^{m}\left(\mathbf{R}^{d}\right)$ denotes the space of locally bounded functions on $\mathbf{R}^{d} \times\left(\mathbf{R}^{d} \backslash\{0\}\right)$ which are $C^{\infty}$ with respect to $\xi$, such that for all $\alpha \in \mathbf{N}^{d}$ the function $x \mapsto \partial_{\xi}^{\alpha} a(x, \xi)$ belongs to $W^{\rho, \infty}\left(\mathbf{R}^{d}\right)$ and there exists a constant $C_{\alpha}>0$ such that

$$
\left\|\partial_{\xi}^{\alpha} a(\cdot, \xi)\right\|_{W^{\rho, \infty}\left(\mathbf{R}^{d}\right)} \leq C_{\alpha}(1+|\xi|)^{m-|\alpha|}, \quad \forall|\xi| \geq \frac{1}{2} .
$$

For such $a$ we set

$$
M_{\rho}^{m}(a)=\sup _{|\alpha| \leq 2 d+2} \sup _{|\xi| \geq \frac{1}{2}}\left\|(1+|\xi|)^{|\alpha|-m} \partial_{\xi}^{\alpha} a(\cdot, \xi)\right\|_{W^{\rho, \infty}\left(\mathbf{R}^{d}\right)} .
$$

Then $\dot{\Gamma}_{\rho}^{m}\left(\mathbf{R}^{d}\right)$ denotes the subspace of $\Gamma_{\rho}^{m}\left(\mathbf{R}^{d}\right)$ which consists of symbols $a(x, \xi)$ which are homogeneous of degree $m$ with respect to $\xi$.

Given a symbol $a$ we denote by $T_{a}$ the associated para-differential operator which is given by the formula

$$
\widehat{T_{a} u}(\xi)=(2 \pi)^{-d} \int_{\mathbf{R}^{d}} \theta(\xi-\eta, \eta) \hat{a}(\xi-\eta, \eta) \psi(\eta) \hat{u}(\eta) d \eta
$$

where $\hat{a}(\zeta, \eta)=\int_{\mathbf{R}^{d}} e^{-i x \cdot \zeta} a(x, \eta) d x$ is the Fourier transform of $a$ with respect to the first variable, $\psi, \theta$ are two fixed $C^{\infty}$ functions on $\mathbf{R}^{d}$ such that for $0<\varepsilon_{1}<\varepsilon_{2}$ small enough

$$
\begin{aligned}
& \psi(\eta)=1 \quad \text { if }|\eta| \geq 1, \quad \psi(\eta)=0 \quad \text { if }|\eta| \leq \frac{1}{2} \\
& \theta(\zeta, \eta)=1 \quad \text { if }|\zeta| \leq \varepsilon_{1}|\eta|, \quad \theta(\zeta, \eta)=0 \quad \text { if }|\zeta| \geq \varepsilon_{2}|\eta| .
\end{aligned}
$$

Notice that if the symbol $a$ is independent of $\xi$ the associated operator $T_{a}$ is called a paraproduct.

Theorem 7.16. Let $m, m^{\prime} \in \mathbf{R}, \rho \in[0,1]$.

(i) If $a \in \Gamma_{0}^{m}\left(\mathbf{R}^{d}\right)$, then for all $\mu \in \mathbf{R} T_{a}$ is continuous from $H_{u l}^{\mu}\left(\mathbf{R}^{d}\right)$ to $H_{u l}^{\mu-m}\left(\mathbf{R}^{d}\right)$ with norm bounded by $C M_{0}^{m}(a)$.

(ii) If $a \in \Gamma_{\rho}^{m}\left(\mathbf{R}^{d}\right), b \in \Gamma_{\rho}^{m^{\prime}}\left(\mathbf{R}^{d}\right)$ then, for all $\mu \in \mathbf{R}, T_{a} T_{b}-T_{a b}$ is continuous from $H_{u l}^{\mu}\left(\mathbf{R}^{d}\right)$ to $H_{u l}^{\mu-m-m^{\prime}+\rho}\left(\mathbf{R}^{d}\right)$ with norm bounded by

$$
C\left(M_{\rho}^{m}(a) M_{0}^{m^{\prime}}(b)+M_{0}^{m}(a) M_{\rho}^{m^{\prime}}(b)\right) .
$$

(iii) Let $a \in \Gamma_{\rho}^{m}\left(\mathbf{R}^{d}\right)$. Denote by $\left(T_{a}\right)^{*}$ the adjoint operator of $T_{a}$ and by $\bar{a}$ the complex conjugate of $a$. Then for all $\mu \in \mathbf{R}\left(T_{a}\right)^{*}-T_{\bar{a}}$ is continuous from $H_{u l}^{\mu}\left(\mathbf{R}^{d}\right)$ to $H_{u l}^{\mu-m+\rho}\left(\mathbf{R}^{d}\right)$ with norm bounded by $C M_{\rho}^{m}(a)$.

Proof. All these points are proved along the same lines. We shall only prove the first one and for simplicity we shall consider symbols in $\dot{\Gamma}_{\rho}^{m}\left(\mathbf{R}^{d}\right)$. We begin by the case where $a$ is a bounded function. Then we write

$$
\chi_{k} T_{a} u=\chi_{k} T_{a}\left(\tilde{\chi}_{k} u\right)+\chi_{k} T_{a}\left(\left(1-\tilde{\chi}_{k}\right) u\right)
$$


where $\tilde{\chi} \in C_{0}^{\infty}\left(\mathbf{R}^{d}\right), \tilde{\chi}=1$ on the support of $\chi$. By the classical theory we have

$$
\left\|\chi_{k} T_{a}\left(\tilde{\chi}_{k} u\right)\right\|_{H^{\mu}} \leq C\|a\|_{L^{\infty}}\left\|\tilde{\chi}_{k} u\right\|_{H^{\mu}} \leq C\|a\|_{L^{\infty}}\|u\|_{H_{u l}^{\mu}} .
$$

Now we write

$$
\chi_{k} T_{a}\left(\left(1-\tilde{\chi}_{k}\right) u\right)=\sum_{j} \chi_{k}\left\{\psi\left(2^{-j} D\right) a\right\}\left\{\varphi\left(2^{-j} D\right)\left(\left(1-\tilde{\chi}_{k}\right) u\right)\right\},
$$

and the desired estimate follows immediately from the first inequality in Lemma 7.13.

We now assume $a(x, \xi)=b(x) h(\xi)$ where $h(\xi)=|\xi|^{m} \tilde{h}\left(\frac{\xi}{|\xi|}\right)$ with $\tilde{h} \in C^{\infty}\left(\mathbf{S}^{d-1}\right)$. Then directly from the definition we have $T_{a}=T_{b} \psi\left(D_{x}\right) h\left(D_{x}\right)$ and our estimate in $(i)$ follows from the first step and from the estimate proved in Proposition 7.9

$$
\|\psi(D) h(D) v\|_{H^{\mu}} \leq C\|\tilde{h}\|_{H^{d+1}\left(\mathbf{S}^{d-1}\right)}\|u\|_{H^{\mu+m}} .
$$

In the last step we introduce $\left(\tilde{h}_{v}\right)_{v \in \mathbf{N}^{*}}$ an orthonormal basis of $L^{2}\left(\mathbf{S}^{d-1}\right)$ consisting of eigenfunctions of the (self adjoint) Laplace Beltrami operator $\Delta_{\omega}=\Delta_{\mathbf{S}^{d-1}}$ on $L^{2}\left(\mathbf{S}^{d-1}\right)$, i.e. $\Delta_{\omega} \tilde{h}_{v}=\lambda_{v}^{2} \tilde{h}_{v}$. By the Weyl formula we know that $\lambda_{v} \sim c v^{\frac{1}{d}}$. Setting $h_{v}=|\xi|^{m} \tilde{h}_{v}(\omega), \omega=\frac{\xi}{|\xi|}$ when $\xi \neq 0$, we can write

$$
a(x, \xi)=\sum_{\nu \in \mathbf{N}^{*}} b_{\nu}(x) h_{\nu}(\xi) \quad \text { where } b_{\nu}(x)=\int_{\mathbf{S}^{d-1}} a(x, \omega) \overline{\tilde{h}_{\nu}(\omega)} d \omega .
$$

Since

$$
\lambda_{\nu}^{2 d+2} b_{v}(x)=\int_{\mathbf{S}^{d-1}} \Delta_{\omega}^{d+1} a(x, \omega) \overline{\tilde{h}_{\nu}(\omega)} d \omega
$$

we deduce that

$$
\left\|b_{v}\right\|_{L^{\infty}\left(\mathbf{R}^{d}\right)} \leq C \lambda_{v}^{-(2 d+2)} M_{0}^{m}(a) .
$$

Moreover there exists a positive constant $K$ such that for all $v \geq 1$

$$
\left\|\tilde{h}_{v}\right\|_{H^{d+1}\left(\mathbf{S}^{d-1}\right)} \leq K \lambda_{v}^{d+1} .
$$

Now using the steps above and Proposition 7.9 we obtain

$$
\begin{aligned}
\left\|T_{a} u\right\|_{H_{u l}^{\mu}} & \leq \sum_{v \geq 1}\left\|T_{b_{v}} \psi\left(D_{x}\right) h_{v}\left(D_{x}\right) u\right\|_{H_{u l}^{\mu}} \\
& \leq C \sum_{v \geq 1}\left\|b_{v}\right\|_{L^{\infty}\left(\mathbf{R}^{d}\right)}\left\|\tilde{h}_{v}\right\|_{H^{d+1}\left(\mathbf{S}^{d-1}\right)}\|u\|_{H_{u l}^{\mu+m}} \\
& \leq C M_{0}^{m}(a)\|u\|_{H_{u l}^{\mu+m}} \sum_{v \geq 1} \lambda_{v}^{-(d+1)}
\end{aligned}
$$

and $\lambda_{\nu}^{-(d+1)} \sim c v^{-\left(1+\frac{1}{d}\right)}$.

\subsubsection{Paraproducts}

We have the following result of paralinearization of a product.

Proposition 7.17. Given two functions $a \in H_{u l}^{\alpha}\left(\mathbf{R}^{d}\right), u \in H_{u l}^{\beta}\left(\mathbf{R}^{d}\right)$ with $\alpha+\beta>0$ we can write

$$
a u=T_{a} u+T_{u} a+R(a, u)
$$

with

$$
\|R(a, u)\|_{H_{u l}^{\alpha+\beta-\frac{d}{2}}\left(\mathbf{R}^{d}\right)} \leq C\|a\|_{H_{u l}^{\alpha}\left(\mathbf{R}^{d}\right)}\|u\|_{H_{u l}^{\beta}\left(\mathbf{R}^{d}\right)} .
$$


Proof. We have

$$
R(a, u)=\sum_{j \geq-1} \sum_{|k-j| \leq 1} \varphi\left(2^{-j} D\right) a \cdot \varphi\left(2^{-k} D\right) u .
$$

We take $\chi \in C_{0}^{\infty}\left(\mathbf{R}^{d}\right)$ satisfying (2.3), $\tilde{\chi} \in C_{0}^{\infty}\left(\mathbf{R}^{d}\right)$ equal to one on the support of $\chi$ and we write $a=\tilde{\chi}_{k} a+(1-$ $\left.\tilde{\chi}_{k}\right) a, u=\tilde{\chi}_{k} u+\left(1-\tilde{\chi}_{k}\right) u$. It follows that

$$
\chi_{k} R(a, u)=\chi_{k} R\left(\tilde{\chi}_{k} a, \tilde{\chi}_{k} u\right)+\chi_{k} S_{k}(a, u) .
$$

The term $\chi_{k} R\left(\widetilde{\chi}_{k} a, \tilde{\chi}_{k} u\right)$ is estimated by the right hand side of (7.23) using Theorem 2.11 in [2]. The remainder $\chi_{k} S_{k}(a, u)$ is estimated using (7.16).

Proposition 7.18. Let $\gamma, r, \mu$ be real numbers such that

$$
r+\mu>0, \quad \gamma \leq r, \quad \gamma<r+\mu-\frac{d}{2} .
$$

There exists a constant $C>0$ such that

$$
\left\|\left(a-T_{a}\right) u\right\|_{H_{u l}^{\gamma}\left(\mathbf{R}^{d}\right)} \leq C\|a\|_{H_{u l}^{r}\left(\mathbf{R}^{d}\right)}\|u\|_{H_{u l}^{\mu}\left(\mathbf{R}^{d}\right)}
$$

whenever the right hand side is finite.

Proof. We write

$$
\begin{aligned}
& \chi_{k}\left(a-T_{a}\right) u=\chi_{k}\left(\tilde{\chi}_{k} a-T_{\widetilde{\chi}_{k}} a\right) \tilde{\chi}_{k} u+R_{1, k} u+R_{2, k} u \\
& R_{1, k} u=\chi_{k}\left(\widetilde{\chi}_{k} a-T_{\widetilde{\chi}_{k}} a\right)\left(1-\widetilde{\chi}_{k}\right) u \\
& R_{2, k}=-\chi_{k} T_{\left(1-\tilde{\chi}_{k}\right) a} u=-\chi_{k} \sum_{j} S_{j}\left(\left(1-\tilde{\chi}_{k}\right) a\right) \Delta_{j}(u)
\end{aligned}
$$

where $\tilde{\chi} \in C_{0}^{\infty}\left(\mathbf{R}^{d}\right)$ is equal to one on the support of $\chi$. According to Proposition 2.12 in [2] we have

$$
\left\|\chi_{k}\left(\tilde{\chi}_{k} a-T_{\widetilde{\chi}_{k}} a\right) \tilde{\chi}_{k} u\right\|_{H^{\gamma}} \leq C\|a\|_{H_{u l}^{r}}\|u\|_{H_{u l}^{\mu}} .
$$

Now

$$
\begin{aligned}
& R_{1, k}=\chi_{k} T_{\left(1-\tilde{\chi}_{k} u\right)} \tilde{\chi}_{k} a+\chi_{k} R\left(1-\tilde{\chi}_{k} u, \tilde{\chi}_{k} a\right) \\
& =\chi_{k} \sum_{j} S_{j}\left(\left(1-\tilde{\chi}_{k}\right) u\right) \Delta_{j}\left(\tilde{\chi}_{k} a\right)+\chi_{k} \sum_{|i-j| \leq 1} \Delta_{i}\left(\left(1-\tilde{\chi}_{k}\right) u\right) \Delta_{j}\left(\tilde{\chi}_{k} a\right) .
\end{aligned}
$$

Therefore we can apply (7.16) in Lemma 7.13 to $R_{1, k}$ and $R_{2, k}$ to conclude that the estimate (7.24) holds for these terms.

\subsection{On transport equations}

We will be using the following result about solutions of vector fields.

Lemma 7.19. Let $I=[0, T], s_{0}>1+\frac{d}{2}$ and $\mu>0$. Then there exists $\mathcal{F}: \mathbf{R}^{+} \rightarrow \mathbf{R}^{+}$non-decreasing such that for $V_{j} \in L^{\infty}\left(I, H^{s_{0}}\left(\mathbf{R}^{d}\right)\right)_{u l} \cap L^{\infty}\left(I, H^{\mu}\left(\mathbf{R}^{d}\right)\right)_{u l} j=1, \ldots, d, f \in L^{1}\left(I, H^{\mu}\left(\mathbf{R}^{d}\right)\right)_{u l}, u_{0} \in H_{u l}^{\mu}\left(\mathbf{R}^{d}\right)$ and any solution $u \in L^{\infty}\left(I, H^{s_{0}}\left(\mathbf{R}^{d}\right)\right)_{u l}$ of the problem

$$
\left(\partial_{t}+V \cdot \nabla_{x}\right) u=f,\left.\quad u\right|_{t=0}=u_{0}
$$

we have 


$$
\begin{aligned}
\|u\|_{L^{\infty}\left(I, H^{\mu}\right)_{u l} \leq} & \mathcal{F}\left(T \| V \| _ { L ^ { \infty } ( I , H ^ { s _ { 0 } } ) _ { u l } ) } \left\{\left\|u_{0}\right\|_{H_{u l}^{\mu}}+\|f\|_{L^{1}\left(I, H^{\mu}\right)_{u l}}\right.\right. \\
& \left.+\sup _{k \in \mathbf{Z}^{d}}\left(\int_{0}^{T}\|u(\sigma)\|_{H_{u l}^{s_{0}}}\left\|\tilde{\chi}_{k} V(\sigma)\right\|_{H^{\mu}} d \sigma\right)\right\}
\end{aligned}
$$

where $\tilde{\chi} \in C_{0}^{\infty}\left(\mathbf{R}^{d}\right)$ is equal to one on the support of $\chi$.

Proof. Set $V_{k}=\tilde{\chi}_{k} V$. We have

$$
\left(\partial_{t}+T_{V_{k}} \cdot \nabla_{x}\right)\left(\chi_{k} u\right)=\chi_{k} f+V_{k} \cdot\left(\nabla_{x} \chi_{k}\right) \tilde{\chi}_{k} u+\left(T_{V_{k}}-V_{k}\right) \cdot \nabla_{x}\left(\chi_{k} u\right)=: g_{k} .
$$

Now computing the quantity $\frac{d}{d t}\left\|\chi_{k} u(t)\right\|_{L^{2}}^{2}$, using the above equation, the fact that $\left\|T_{V} \cdot \nabla_{x}+\left(T_{V} \cdot \nabla_{x}\right)^{*}\right\|_{L^{2} \rightarrow L^{2}} \leq$ $C\|V(t)\|_{W^{1, \infty}}$ and the Gronwall inequality we obtain

$$
\left\|\chi_{k} u(t)\right\|_{L^{2}} \leq \mathcal{F}\left(\|V\|_{L^{1}\left(I, W^{1, \infty}\right)}\right)\left\{\left\|\chi_{k} u_{0}\right\|_{L^{2}}+\int_{0}^{t}\left\|g_{k}(\sigma)\right\|_{L^{2}} d \sigma\right\} .
$$

Now we can write

$$
\left(\partial_{t}+T_{V_{k}} \cdot \nabla_{x}\right)\left\langle D_{x}\right\rangle^{\mu}\left(\chi_{k} u\right)=\left\langle D_{x}\right\rangle^{\mu} g_{k}+\left[T_{V_{k}},\left\langle D_{x}\right\rangle^{\mu}\right] \cdot \nabla_{x}\left(\chi_{k} u\right) .
$$

By the symbolic calculus (see Theorem 7.16(ii)) we have

$$
\left\|\left[T_{V_{k}},\left\langle D_{x}\right\rangle^{\mu}\right] \cdot \nabla_{x}\left(\chi_{k} u\right)(t)\right\|_{L^{2}} \leq C\|V(t)\|_{W^{1, \infty}}\left\|\chi_{k} u(t)\right\|_{H^{\mu}} .
$$

Therefore using (7.30) and the Gronwall inequality we obtain

$$
\left\|\chi_{k} u(t)\right\|_{H^{\mu}} \leq \mathcal{F}\left(\|V\|_{L^{1}\left(I, W^{1, \infty}\right)}\right)\left\{\left\|\chi_{k} u_{0}\right\|_{H^{\mu}}+\int_{0}^{t}\left\|g_{k}(\sigma)\right\|_{H^{\mu}} d \sigma\right\} .
$$

Coming back to the definition of $g$ given in (7.29) we have

$$
\left\|V_{k} \cdot\left(\nabla_{x} \chi_{k}\right) \tilde{\chi}_{k} u(t)\right\|_{H^{\mu}} \leq C\left(\|V(t)\|_{L^{\infty}}\left\|\tilde{\chi}_{k} u(t)\right\|_{H^{\mu}}+\|u(t)\|_{L^{\infty}}\left\|V_{k}(t)\right\|_{H^{\mu}}\right) .
$$

On the other hand we have

$$
\left(V_{k}-T_{V_{k}}\right) \cdot \nabla_{x}\left(\chi_{k} u\right)=T_{\nabla_{x}\left(\chi_{k} u\right)} \cdot V_{k}+R\left(V_{k}, \nabla_{x}\left(\chi_{k} u\right)\right) .
$$

By Theorem 7.16(i) and an easy computation we see that

$$
\left\|T_{\nabla_{x}\left(\chi_{k} u\right)(t)} \cdot V_{k}(t)\right\|_{H^{\mu}}+\left\|R\left(V_{k}, \nabla_{x}\left(\chi_{k} u\right)\right)\right\| \leq C\|u(t)\|_{W^{1, \infty}}\left\|V_{k}(t)\right\|_{H^{\mu}} .
$$

Using (7.31), the Gronwall inequality, the embedding of $H_{u l}^{s_{0}}$ in $W^{1, \infty}$ and the above estimates we obtain the desired conclusion.

\subsection{Commutation with a vector field}

Lemma 7.20. Let $I=[0, T], V \in C^{0}\left(I, W^{1+\varepsilon, \infty}\left(\mathbf{R}^{d}\right)\right)$ for some $\varepsilon>0$ and consider a symbol $p=p(t, x, \xi)$ which is homogeneous of order $m$. Then there exists a positive constant $K$ (independent of $p, V$ ) such that for any $t \in I$ and any $u \in C^{0}\left(I, H_{u l}^{m}\left(\mathbf{R}^{d}\right)\right)$ we have

$$
\left\|\left[T_{p}, \partial_{t}+T_{V} \cdot \nabla_{x}\right] u(t, \cdot)\right\|_{L_{u l}^{2}\left(\mathbf{R}^{d}\right)} \leq K C(p, V)\|u(t, \cdot)\|_{H_{u l}^{m}\left(\mathbf{R}^{d}\right)}
$$

where

$$
C(p, V):=M_{0}^{m}(p)\|V\|_{C^{0}\left(I, W^{1+\varepsilon, \infty}\left(\mathbf{R}^{d}\right)\right)}+M_{0}^{m}\left(\partial_{t} p+V \cdot \nabla_{x} p\right)\|V\|_{L^{\infty}\left(I \times \mathbf{R}^{d}\right)} .
$$


Proof. We proceed as in the proof of Theorem 7.16 and we begin by the case where $m=0$ and $p$ is a function. We denote by $\mathcal{R}$ the set of continuous operators $R(t)$ from $L_{u l}^{2}\left(\mathbf{R}^{d}\right)$ to $L^{2}\left(\mathbf{R}^{d}\right)$ such that $\sup _{t \in I}\|R(t) u(t)\|_{L^{2}\left(\mathbf{R}^{d}\right)}$ is bounded by the right hand side of (7.32). We write

$$
\chi_{k}\left[T_{p}, \partial_{t}+T_{V} \cdot \nabla_{x}\right]=\chi_{k}\left[T_{p}, \partial_{t}+T_{V} \cdot \nabla_{x}\right] \tilde{\chi}_{k}+\chi_{k}\left[T_{p}, \partial_{t}+T_{V} \cdot \nabla_{x}\right]\left(1-\tilde{\chi}_{k}\right)
$$

where $\tilde{\chi} \in C_{0}^{\infty}\left(\mathbf{R}^{d}\right)$ is equal to one on the support of $\chi$. By Lemma 2.17 in [2] the first operator in the right hand side of the above equality belongs to $\mathcal{R}$. Let us look at the second term. It is equal to

$$
-\chi_{k} T_{\partial_{t}}\left(1-\tilde{\chi}_{k}\right)+\chi_{k} T_{p} T_{V} \cdot \nabla_{x}\left(1-\tilde{\chi}_{k}\right)-\chi_{k} T_{V} \cdot \nabla_{x} T_{p}\left(1-\tilde{\chi}_{k}\right)=: A+B+C .
$$

We can write

$$
A=-\chi_{k} T_{\partial_{t} p+V \cdot \nabla_{x} p}\left(1-\tilde{\chi}_{k}\right)+\chi_{k} T_{V \cdot \nabla_{x} p}\left(1-\tilde{\chi}_{k}\right)=: A_{1}+A_{2} .
$$

By Theorem 7.16(i) the term $A_{1}$ belongs to $\mathcal{R}$. Now

$$
\begin{aligned}
A_{2} u & =\chi_{k} T_{\operatorname{div}(p V)-p \operatorname{div} V\left(1-\tilde{\chi}_{k}\right) u} \\
& =\sum_{j \geq-1} \psi\left(2^{-j} D\right)(\operatorname{div}(p V)-p \operatorname{div} V) \chi_{k} \varphi\left(2^{-j} D\right)\left(\left(1-\tilde{\chi}_{k}\right) u\right) .
\end{aligned}
$$

Since

$$
\left\|\psi\left(2^{-j} D_{x}\right)(\operatorname{div}(p V)-p \operatorname{div} V)\right\|_{L^{\infty}} \leq C 2^{j}\|p\|_{L^{\infty}}\|V\|_{W^{1, \infty}}
$$

we deduce from Remark 7.14 that $A_{2} \in \mathcal{R}$.

Let $\underline{\chi} \in C_{0}^{\infty}\left(\mathbf{R}^{d}\right)$ such that $\tilde{\chi}=1$ on the support of $\underline{\chi}$ and $\underline{\chi}=1$ on the support of $\chi$. We write

$$
B=\chi_{k} T_{p} \underline{\chi}_{k} T_{V} \cdot \nabla_{x}\left(1-\tilde{\chi}_{k}\right)+\chi_{k} T_{p}\left(1-\underline{\chi}_{k}\right) T_{V} \cdot \nabla_{x}\left(1-\tilde{\chi}_{k}\right)=: B_{1}+B_{2} .
$$

By Theorem 7.16(i) we have

$$
\begin{aligned}
\left\|B_{1} u\right\|_{L^{2}} & \leq C\|p\|_{L^{\infty}}\left\|\underline{\chi}_{k} T_{V} \cdot \nabla_{x}\left(1-\tilde{\chi}_{k}\right) u\right\|_{L^{2}} \\
& \leq C\|p\|_{L^{\infty}} \sum_{j \geq-1}\left\|\left(\psi\left(2^{-j} D_{x}\right) V\right) 2^{j} \underline{\chi}_{k} \varphi_{1}\left(2^{-j} D\right)\left(1-\tilde{\chi}_{k}\right) u\right\|_{L^{2}} \\
& \leq C\|p\|_{L^{\infty}}\|V\|_{L^{\infty}} \sum_{j \geq-1} 2^{j}\left\|\underline{\chi}_{k} \varphi_{1}\left(2^{-j} D\right)\left(1-\tilde{\chi}_{k}\right) u\right\|_{L^{2}}
\end{aligned}
$$

and Remark 7.14 shows that $B_{1} \in \mathcal{R}$.

Now by (7.15) and Theorem 7.16 we can write

$$
\begin{aligned}
\left\|B_{2} u\right\|_{L^{2}} & \leq C\|p\|_{L^{\infty}}\left\|T_{V} \cdot \nabla_{x}\left(1-\tilde{\chi}_{k}\right) u\right\|_{H_{u l}^{-1}} \\
& \leq C\|p\|_{L^{\infty}}\|V\|_{L^{\infty}}\|u\|_{L_{u l}^{2}}
\end{aligned}
$$

so $B_{2} \in \mathcal{R}$. The term $C$ is estimated exactly by the same way, introducing a cut-off $\underline{\chi_{k}}$ after the operator $T_{V} \cdot \nabla_{x}$. Thus $C \in \mathcal{R}$.

The case where $p=a(t, x) h(\xi)$ and then were $p$ is a general homogeneous symbol of order $m$ is handled as in the proof of Theorem 7.16.

\section{Conflict of interest statement}

None declared.

\section{Acknowledgements}

We would like to thank the referees for their comments which lead to significant improvements in the presentation of the paper. 


\section{Appendix A}

Let $\alpha \in(0,+\infty), \alpha \neq 1$ and $S(t)=e^{-i t\left|D_{x}\right|^{\alpha}}$. Our aim is to prove the following result.

Proposition A.1. Let $s, \sigma \in \mathbf{R}$. Assume that there exists $t_{0} \neq 0$ such that $S\left(t_{0}\right)$ is continuous from $C_{*}^{\sigma}\left(\mathbf{R}^{d}\right)$ to $C_{*}^{s}\left(\mathbf{R}^{d}\right)$. Then $s \leq \sigma-\frac{d \alpha}{2}$.

Proof. Without loss of generality we can assume that $t_{0}=-1$. Our hypothesis reads

$$
\exists C>0: \quad\|S(-1) u\|_{C_{*}^{s}\left(\mathbf{R}^{d}\right)} \leq C\|u\|_{C_{*}^{\sigma}\left(\mathbf{R}^{d}\right)}, \quad \forall u \in C_{*}^{\sigma}\left(\mathbf{R}^{d}\right) .
$$

Now if $u \in L^{\infty}\left(\mathbf{R}^{d}\right)$ we set $\widehat{\Delta_{j}} u(\xi)=\varphi\left(2^{-j} \xi\right) \hat{u}(\xi)$, where $\varphi \in C_{0}^{\infty}\left(\mathbf{R}^{d}\right)$, with supp $\varphi \subset\left\{\xi: \frac{1}{2} \leq|\xi| \leq 2\right\}$. Then for fixed $j \in \mathbf{N}$ we have $\Delta_{j} u \in C_{*}^{\sigma}\left(\mathbf{R}^{d}\right)$ and

$$
\left\|\Delta_{j} u\right\|_{C_{*}^{\sigma}\left(\mathbf{R}^{d}\right)} \leq C 2^{j \sigma}\left\|\Delta_{j} u\right\|_{L^{\infty}\left(\mathbf{R}^{d}\right)} \leq C^{\prime} 2^{j \sigma}\|u\|_{L^{\infty}\left(\mathbf{R}^{d}\right)} .
$$

This follows from the fact that $\left\|\Delta_{j} u\right\|_{C_{*}^{\sigma}\left(\mathbf{R}^{d}\right)}=\sup _{k \in \mathbf{N}} 2^{k \sigma}\left\|\Delta_{k} \Delta_{j} u\right\|_{L^{\infty}\left(\mathbf{R}^{d}\right)}$ and the fact that $\Delta_{k} \Delta_{j}=0$ if $|j-k| \geq 2$. Since $\Delta_{j}$ commutes with $S(-1)$, we see that

$$
2^{j s}\left\|S(-1) \Delta_{j} \Delta_{j} u\right\|_{L^{\infty}\left(\mathbf{R}^{d}\right)} \leq\left\|S(-1) \Delta_{j} u\right\|_{C_{*}^{s}\left(\mathbf{R}^{d}\right)} .
$$

It follows from (A.1) applied to $\Delta_{j} u$ with $u \in L^{\infty}\left(\mathbf{R}^{d}\right)$ that one can find a positive constant $C$ such that

$$
2^{j s}\left\|S(-1) \Delta_{j} \Delta_{j} u\right\|_{L^{\infty}\left(\mathbf{R}^{d}\right)} \leq C 2^{j \sigma}\|u\|_{L^{\infty}\left(\mathbf{R}^{d}\right)} \quad \forall u \in L^{\infty}\left(\mathbf{R}^{d}\right), \forall j \in \mathbf{N} .
$$

Let us set $T_{j}=S(-1) \Delta_{j} \Delta_{j}$. Then (A.2) shows that

$$
\left\|T_{j}\right\|_{L^{\infty} \rightarrow L^{\infty}} \leq C 2^{j(\sigma-s)} \text {. }
$$

Now

$$
T_{j} u(x)=(2 \pi)^{-d} \iint e^{i\left[(x-y) \cdot \xi+|\xi|^{\alpha}\right]} \varphi^{2}\left(2^{-j} \xi\right) u(y) d y d \xi .
$$

We shall set $h=2^{-j}$ and take $j$ large enough. Then setting $\eta=h \xi$ we obtain

$$
T_{j} u(x)=\int_{\mathbf{R}^{d}} K_{h}(x-y) u(y) d y
$$

where

$$
K_{h}(z)=(2 \pi h)^{-d} \int_{\mathbf{R}^{d}} e^{\frac{i}{h}\left(z \cdot \eta+h^{1-\alpha}|\eta|^{\alpha}\right)} \varphi^{2}(\eta) d \eta .
$$

We shall use the following well known lemma.

Lemma A.2. Let $K \in C^{0}\left(\mathbf{R}^{d} \times \mathbf{R}^{d}\right)$ be such that $\sup _{x \in \mathbf{R}^{d}} \int|K(x, y)| d y<+\infty$. Then the operator $T$ defined by $T u(x)=\int K(x, y) u(y) d y$ is continuous from $L^{\infty}\left(\mathbf{R}^{d}\right)$ to $L^{\infty}\left(\mathbf{R}^{d}\right)$ and $\|T\|_{L^{\infty} \rightarrow L^{\infty}}=\sup _{x \in \mathbf{R}^{d}} \int|K(x, y)| d y$.

It follows from this lemma that in our case we have

$$
\left\|T_{j}\right\|_{L^{\infty} \rightarrow L^{\infty}}=\int_{\mathbf{R}^{d}}\left|K_{h}(z)\right| d z .
$$

Setting $z=h^{1-\alpha} s$ and $\widetilde{K}_{h}(s)=K_{h}\left(h^{1-\alpha} s\right)$ we find that

$$
\left\|T_{j}\right\|_{L^{\infty} \rightarrow L^{\infty}}=h^{d(1-\alpha)} \int_{\mathbf{R}^{d}}\left|\widetilde{K}_{h}(s)\right| d s
$$


with

$$
\widetilde{K}_{h}(s)=(2 \pi h)^{-d} \int_{\mathbf{R}^{d}} e^{i h^{-\alpha} \phi(s, \eta)} \varphi^{2}(\eta) d \eta, \quad \phi(s, \eta)=s \cdot \eta+|\eta|^{\alpha} .
$$

Recall that $\operatorname{supp} \varphi \subset\left\{\eta: \frac{1}{2} \leq|\eta| \leq 2\right\}$. We have $\frac{\partial \phi}{\partial \eta}=s+\alpha \frac{\eta}{|\eta|^{2-\alpha}}$.

Case 1: $|s| \leq \frac{1}{2} \frac{\alpha}{2^{|1-\alpha|}}$. Here, on the support of $\varphi$, we have

$$
\left|\frac{\partial \phi}{\partial \eta}(s, \eta)\right| \geq \frac{\alpha}{|\eta|^{1-\alpha}}-|s| \geq \frac{1}{2} \frac{\alpha}{2^{|1-\alpha|}} .
$$

Therefore integrating by parts in the right hand side of (A.5) using the vector field $L=\frac{h^{\alpha}}{i} \frac{1}{\left|\partial_{\eta} \phi\right|^{2}} \sum_{k=1}^{d} \frac{\partial \phi}{\partial \eta_{k}} \frac{\partial}{\partial \eta_{k}}$ we obtain

$$
\left|\widetilde{K}_{h}(s)\right| \leq C_{N} h^{N}, \quad \forall N \in \mathbf{N} .
$$

Case $2:|s| \geq 2^{1+|\alpha-1|} \alpha$. On the support of $\varphi$ we have

$$
\left|\frac{\partial \phi}{\partial \eta}(s, \eta)\right| \geq|s|-\frac{\alpha}{|\eta|^{1-\alpha}} \geq 2^{|\alpha-1|} \alpha .
$$

Then using the same vector field as in the first case and noticing that $\partial_{\eta}^{\alpha} \phi$ is independent of $s$ when $|\alpha| \geq 2$ we obtain

$$
\left|\widetilde{K}_{h}(s)\right| \leq C_{N}|s|^{-N} h^{N}, \quad \forall N \in \mathbf{N} .
$$

Case 3: $\frac{1}{2} \frac{\alpha}{2^{|1-\alpha|}} \leq|s| \leq 2^{1+|\alpha-1|} \alpha$. Here the function $\phi$ has a critical point given by $\frac{\eta}{|\eta|^{2-\alpha}}=-\frac{s}{\alpha}$. It follows that $\frac{1}{|\eta|^{1-\alpha}}=\frac{|s|}{\alpha}$, which implies that $\eta_{c}=c_{\alpha} s|s|^{\frac{2-\alpha}{\alpha-1}}$. Moreover we have

$$
\frac{\partial^{2} \phi}{\partial \eta_{j} \partial \eta_{k}}=\alpha|\eta|^{\alpha-2} m_{j k}, \quad m_{j k}=\delta_{j k}-(\alpha-2) \omega_{j} \omega_{k}, \quad \omega=\frac{\eta}{|\eta|} .
$$

Since $\operatorname{det}\left(m_{j k}\right)=c_{0} \neq 0$ we obtain $\left(\left|\operatorname{det}\left(\frac{\partial^{2} \phi}{\partial \eta_{j} \partial \eta_{k}}\left(s, \eta_{c}\right)\right)\right|\right)^{\frac{1}{2}}=c_{\alpha, d}|s|^{\frac{(\alpha-2) d}{2(\alpha-1)}}$. The stationary phase formula implies that there exists $C_{d}>0$ such that

$$
\widetilde{K}_{h}(s)=C_{\alpha, d} h^{-d} h^{\frac{\alpha d}{2}}\left\{\frac{e^{i h^{-\alpha} \phi\left(s, \eta_{c}\right)}}{|s|^{\frac{(\alpha-2) d}{2(\alpha-1)}}} \varphi^{2}\left(\eta_{c}\right)+\mathcal{O}\left(h^{\alpha}\right)\right\} .
$$

Using (A.4), (A.6), (A.7), (A.8) we can conclude that for $j$ large enough we have

$$
\left\|T_{j}\right\|_{L^{\infty} \rightarrow L^{\infty}} \geq C h^{d(1-\alpha)} h^{-d} h^{\frac{d \alpha}{2}}-C_{N} h^{N} \geq C^{\prime} h^{-\frac{d \alpha}{2}} .
$$

Recalling that $h=2^{-j}$ we obtain

$$
\left\|T_{j}\right\|_{L^{\infty} \rightarrow L^{\infty}} \geq C^{\prime} 2^{j \frac{d \alpha}{2}} .
$$

Then Proposition A.1 follows from (A.3) and (A.9).

\section{References}

[1] Thomas Alazard, Nicolas Burq, Claude Zuily, On the water-wave equations with surface tension, Duke Math. J. 158 (3) (2011) $413-499$.

[2] Thomas Alazard, Nicolas Burq, Claude Zuily, On the Cauchy problem for gravity water waves, Invent. Math. 198 (1) (2014) $71-163$.

[3] Thomas Alazard, Nicolas Burq, Claude Zuily, Strichartz estimates and the Cauchy problem for the gravity water waves equations, arXiv: 1404.4276.

[4] Thomas Alazard, Guy Métivier, Paralinearization of the Dirichlet to Neumann operator, and regularity of three-dimensional water waves, Commun. Partial Differ. Equ. 34 (10-12) (2009) 1632-1704.

[5] Serge Alinhac, Paracomposition et opérateurs paradifférentiels, Commun. Partial Differ. Equ. 11 (1) (1986) 87-121.

[6] Serge Alinhac, Existence d'ondes de raréfaction pour des systèmes quasi-linéaires hyperboliques multidimensionnels, Commun. Partial Differ Equ. 14 (2) (1989) 173-230. 
[7] Claude Bardos, David Lannes, Mathematics for 2d interfaces, in: C. Josserand, L. Saint-Raymond (Eds.), Singularities in Mechanics: Formation, Propagation and Microscopic Description, in: Panor. Synth., vol. 38, 2012, xxxiv+162 pp.

[8] Jean-Michel Bony, Calcul symbolique et propagation des singularités pour les équations aux dérivées partielles non linéaires, Ann. Sci. Éc. Norm. Supér. (4) 14 (2) (1981) 209-246.

[9] Joseph Boussinesq, Sur une importante simplification de la théorie des ondes que produisent, à la surface d'un liquide, l'emersion d'un solide ou l'impulsion d'un coup de vent, Ann. Sci. Éc. Norm. Supér. (3) 27 (1910) 9-42.

[10] Angel Castro, Diego Córdoba, Charles Fefferman, Francisco Gancedo, Javier Gómez-Serrano, Splash singularity for water waves, Proc. Natl. Acad. Sci. 109 (3) (January 17, 2012) 733-738.

[11] Angel Castro, David Lannes, Well-posedness and shallow-water stability for a new Hamiltonian formulation of the water waves equations with vorticity, arXiv:1402.0464.

[12] Walter Craig, An existence theory for water waves and the Boussinesq and Korteweg-de Vries scaling limits, Commun. Partial Differ. Equ. 10 (8) (1985) 787-1003.

[13] Walter Craig, David P. Nicholls, Travelling two and three dimensional capillary gravity water waves, SIAM J. Math. Anal. 32 (2) (2000) 323-359 (electronic).

[14] W. Craig, C. Sulem, P.-L. Sulem, Nonlinear modulation of gravity waves: a rigorous approach, Nonlinearity 5 (2) (1992) $497-522$.

[15] A.-L. Dalibard, C. Prange, Well-posedness of the Stokes-Coriolis system in the half-space over a rough surface, arXiv:1304.6651.

[16] H. Favre, Etude théorique et expérimentale des ondes de translation dans les canaux découverts, Dunod, Paris, 1935.

[17] Charles Fefferman, Elias Stein, $H^{p}$ spaces of several variables, Acta Math. 129 (1) (1972) 137-193.

[18] David Gérard-Varet, Nader Masmoudi, Relevance of the slip condition for fluid flows near an irregular boundary, Commun. Math. Phys. 295 (1) (2010) 99-137.

[19] Pierre Germain, Nader Masmoudi, Jalal Shatah, Global solutions for the gravity water waves equation in dimension 3, Ann. Math. (2) 175 (2) (2012) 691-754.

[20] Pierre Grisvard, Elliptic Problems in Non Smooth Domains, Pitman, 1985.

[21] Gérard Iooss, Pavel I. Plotnikov, Small divisor problem in the theory of three-dimensional water gravity waves, Mem. Am. Math. Soc. 200 (940) (2009), viii+128 pp.

[22] Tosio Kato, The Cauchy problem for quasi-linear symmetric hyperbolic systems, Arch. Ration. Mech. Anal. 58 (3) (1975) $181-205$.

[23] David Lannes, The Water Waves Problem: Mathematical Analysis and Asymptotics, Math. Surv. Monogr., vol. 188, American Mathematical Society, Providence, RI, 2013, xx+321 pp.

[24] David Lannes, Well-posedness of the water-waves equations, J. Am. Math. Soc. 18 (3) (2005) 605-654 (electronic).

[25] David Lannes, A stability criterion for two-fluid interfaces and applications, Arch. Ration. Mech. Anal. 208 (2) (2013) $481-567$.

[26] Hans Lindblad, Well-posedness for the motion of an incompressible liquid with free surface boundary, Ann. Math. (2) 162 (1) (2005) $109-194$.

[27] Jacques-Louis Lions, Enrico Magenes, Problèmes aux limites non homogènes, vol. 1, Dunod, 1968.

[28] Guy Metivier, Para-differential Calculus and Applications to the Cauchy Problem for Nonlinear Systems, Centro di Ricerca Matematica Ennio De Giorgi (CRM) Series, vol. 5, Edizioni della Normale, Pisa, 2008.

[29] John Reeder, Marvin Shinbrot, Three-dimensional, nonlinear wave interaction in water of constant depth, Nonlinear Anal. 5 (3) (1981) 303-323.

[30] Sijue Wu, Well-posedness in Sobolev spaces of the full water wave problem in 3-D, J. Am. Math. Soc. 12 (2) (1999) 445-495.

[31] Sijue Wu, Almost global well-posedness of the 2-D full water wave problem, Invent. Math. 177 (1) (2009) 45-135.

[32] Sijue Wu, Global wellposedness of the 3-D full water wave problem, Invent. Math. 184 (1) (2011) 125-220.

[33] Vladimir E. Zakharov, Stability of periodic waves of finite amplitude on the surface of a deep fluid, J. Appl. Mech. Tech. Phys. 9 (2) (1968) 190-194. 